RIMS-1759

INTER-UNIVERSAL TEICHMÜLLER THEORY IV:

LOG-VOLUME COMPUTATIONS AND SET-THEORETIC FOUNDATIONS

By

Shinichi MOCHIZUKI

August 2012

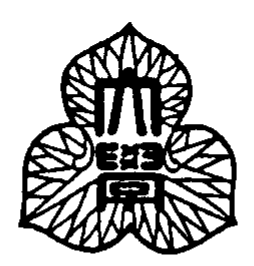

京都大学 数理解析研究所

RESEARCH INSTITUTE FOR MATHEMATICAL SCIENCES

KYOTO UNIVERSITY, Kyoto, Japan 


\title{
INTER-UNIVERSAL TEICHMÜLLER THEORY IV: LOG-VOLUME COMPUTATIONS AND SET-THEORETIC FOUNDATIONS
}

\author{
SHINichi Mochizuki
}

August 2012

\begin{abstract}
.
The present paper forms the fourth and final paper in a series of papers concerning "inter-universal Teichmüller theory". In the first three papers of the series, we introduced and studied the theory surrounding the log-theta-lattice, a highly non-commutative two-dimensional diagram of "miniature models of conventional scheme theory", called $\Theta^{ \pm \mathrm{ell}} \mathrm{NF}$-Hodge theaters, that were associated, in the first paper of the series, to certain data, called initial $\Theta$-data. This data includes an elliptic curve $E_{F}$ over a number field $F$, together with a prime number $l \geq 5$. Consideration of various properties of the log-theta-lattice led naturally to the establishment, in the third paper of the series, of multiradial algorithms for constructing "splitting monoids of LGP-monoids". Here, we recall that "multiradial algorithms" are algorithms that make sense from the point of view of an "alien arithmetic holomorphic structure", i.e., the ring/scheme structure of a $\Theta^{ \pm \text {ell }} \mathrm{NF}$-Hodge theater related to a given $\Theta^{ \pm \text {ell }} \mathrm{NF}$-Hodge theater by means of a non-ring/scheme-theoretic horizontal arrow of the log-theta-lattice. In the present paper, estimates arising from these multiradial algorithms for splitting monoids of LGP-monoids are applied to verify various diophantine results which imply, for instance, the so-called Vojta Conjecture for hyperbolic curves, the ABC Conjecture, and the Szpiro Conjecture for elliptic curves. Finally, we examine the foundational/set-theoretic issues surrounding the vertical and horizontal arrows of the log-theta-lattice by introducing and studying the basic properties of the notion of a "species", which may be thought of as a sort of formalization, via set-theoretic formulas, of the intuitive notion of a "type of mathematical object". These foundational issues are closely related to the central role played in the present series of papers by various results from absolute anabelian geometry, as well as to the idea of gluing together distinct models of conventional scheme theory, i.e., in a fashion that lies outside the framework of conventional scheme theory. Moreover, it is precisely these foundational issues surrounding the vertical and horizontal arrows of the log-theta-lattice that led naturally to the introduction of the term "interuniversal".
\end{abstract}

\section{Contents:}

Introduction

$\S 0$. Notations and Conventions

$\S 1$. Log-volume Estimates

$\S 2$. Diophantine Inequalities

$\S 3$. Inter-universal Formalism: the Language of Species

Typeset by $\mathcal{A M S}_{\mathcal{M}}-\mathrm{T}_{\mathrm{EX}}$ 


\section{Introduction}

The present paper forms the fourth and final paper in a series of papers concerning "inter-universal Teichmüller theory". In the first three papers, [IUTchI], [IUTchII], and [IUTchIII], of the series, we introduced and studied the theory surrounding the log-theta-lattice [cf. the discussion of [IUTchIII], Introduction], a highly non-commutative two-dimensional diagram of "miniature models of conventional scheme theory", called $\Theta^{ \pm \text {ell }} N F$-Hodge theaters, that were associated, in the first paper [IUTchI] of the series, to certain data, called initial $\Theta$-data. This data includes an elliptic curve $E_{F}$ over a number field $F$, together with a prime number $l \geq 5$ [cf. [IUTchI], $§ \mathrm{I} 1$ ]. Consideration of various properties of the log-theta-lattice leads naturally to the establishment of multiradial algorithms for constructing "splitting monoids of LGP-monoids" [cf. [IUTchIII], Theorem A]. Here, we recall that "multiradial algorithms" [cf. the discussion of [IUTchIII], Introduction] are algorithms that make sense from the point of view of an "alien arithmetic holomorphic structure", i.e., the ring/scheme structure of a $\Theta^{ \pm \text {ell }}$ NF-Hodge theater related to a given $\Theta^{ \pm \text {ell }} \mathrm{NF}$-Hodge theater by means of a non-ring/schemetheoretic horizontal arrow of the log-theta-lattice. In the final portion of [IUTchIII], by applying these multiradial algorithms for splitting monoids of LGP-monoids, we obtained estimates for the log-volume of these LGP-monoids [cf. [IUTchIII], Theorem B]. In the present paper, these estimates will be applied to verify various diophantine results.

In $\S 1$ of the present paper, we start by discussing various elementary estimates for the log-volume of various tensor products of the modules obtained by applying the p-adic logarithm to the local units - i.e., in the terminology of [IUTchIII], "tensor packets of log-shells" [cf. the discussion of [IUTchIII], Introduction] — in terms of various well-known invariants, such as differents, associated to a mixedcharacteristic nonarchimedean local field [cf. Propositions 1.1, 1.2, 1.3, 1.4]. We then discuss similar — but technically much simpler! — log-volume estimates in the case of complex archimedean local fields [cf. Proposition 1.5]. After reviewing a certain classical estimate concerning the distribution of prime numbers [cf. Proposition 1.6], as well as some elementary general nonsense concerning weighted averages [cf. Proposition 1.7] and well-known elementary facts concerning elliptic curves [cf. Proposition 1.8], we then proceed to compute explicitly, in more elementary language, the quantity that was estimated in [IUTchIII], Theorem B. These computations yield a quite strong/explicit diophantine inequality [cf. Theorem 1.10] concerning elliptic curves that are in "sufficiently general position", so that one may apply the general theory developed in the first three papers of the series.

In $\S 2$ of the present paper, after reviewing another classical estimate concerning the distribution of prime numbers [cf. Proposition 2.1, (ii)], we then proceed to apply the theory of [GenEll] to reduce various diophantine results concerning an arbitrary elliptic curve over a number field to results of the type obtained in Theorem 1.10 concerning elliptic curves that are in "sufficiently general position" [cf. Corollary 2.2; the discussion of Remark 2.3.2, (ii)]. This reduction allows us to derive the following result [cf. Corollary 2.3], which constitutes the main application of the "inter-universal Teichmüller theory" developed in the present series of papers. 
Theorem A. (Diophantine Inequalities) Let $X$ be a smooth, proper, geometrically connected curve over a number field; $D \subseteq X$ a reduced divisor; $U_{X} \stackrel{\text { def }}{=} X \backslash D$; $d$ a positive integer; $\epsilon \in \mathbb{R}_{>0}$ a positive real number. Write $\omega_{X}$ for the canonical sheaf on $X$. Suppose that $U_{X}$ is a hyperbolic curve, i.e., that the degree of the line bundle $\omega_{X}(D)$ is positive. Then, relative to the notation of [GenEll] [reviewed in the discussion preceding Corollary 2.2 of the present paper], one has an inequality of "bounded discrepancy classes"

$$
\mathrm{ht}_{\omega_{X}(D)} \lesssim(1+\epsilon)\left(\log -\operatorname{diff}_{X}+\log -\operatorname{cond}_{D}\right)
$$

of functions on $U_{X}(\overline{\mathbb{Q}}) \leq d-i . e$. , the function $(1+\epsilon)\left(\log\right.$-diff ${ }_{X}+\log$-cond $\left.{ }_{D}\right)-$ ht $_{\omega_{X}(D)}$ is bounded below by a constant on $U_{X}(\overline{\mathbb{Q}}) \leq d$ [cf. [GenEll], Definition 1.2, (ii), as well as Remark 2.3.1, (ii), of the present paper].

Thus, Theorem A asserts an inequality concerning the canonical height [i.e., "ht $\omega_{\omega_{X}}(D)$ "], the logarithmic different [i.e., "log-diff ${ }_{X}$ "], and the logarithmic conductor [i.e., "log-cond ${ }_{D}$ "] of points of the curve $U_{X}$ valued in number fields whose extension degree over $\mathbb{Q}$ is $\leq d$. In particular, the so-called Vojta Conjecture for hyperbolic curves, the ABC Conjecture, and the Szpiro Conjecture for elliptic curves all follow as special cases of Theorem A. We refer to [Vjt] for a detailed exposition of these conjectures.

Finally, in $\S 3$, we examine certain foundational issues underlying the theory of the present series of papers. Typically in mathematical discussions - such as, for instance, the theory developed in the present series of papers! — one defines various "types of mathematical objects" [i.e., such as groups, topological spaces, or schemes], together with a notion of "morphisms" between two particular examples of a specific type of mathematical object [i.e., morphisms between groups, between topological spaces, or between schemes]. Such objects and morphisms [typically] determine a category. On the other hand, if one restricts one's attention to such a category, then one must keep in mind the fact that the structure of the category - i.e., which consists only of a collection of objects and morphisms satisfying certain properties! - does not include any mention of the various sets and conditions satisfied by those sets that give rise to the "type of mathematical object" under consideration. For instance, the data consisting of the underlying set of a group, the group multiplication law on the group, and the properties satisfied by this group multiplication law cannot be recovered [at least in an a priori sense!] from the structure of the "category of groups". Put another way, although the notion of a "type of mathematical object" may give rise to a "category of such objects", the notion of a "type of mathematical object" is much stronger - in the sense that it involves much more mathematical structure - than the notion of a category. Indeed, a given "type of mathematical object" may have a very complicated internal structure, but may give rise to a category equivalent to a one-morphism category [i.e., a category with precisely one morphism]; in particular, in such cases, the structure of the associated category does not retain any information of interest concerning the internal structure of the "type of mathematical object" under consideration.

In Definition 3.1, (iii), we formalize this intuitive notion of a "type of mathematical object" by defining the notion of a species as, roughly speaking, a collection 
of set-theoretic formulas that gives rise to a category in any given model of set theory [cf. Definition 3.1, (iv)], but, unlike any specific category [e.g., of groups, etc.] is not confined to any specific model of set theory. In a similar vein, by working with collections of set-theoretic formulas, one may define a species-theoretic analogue of the notion of a functor, which we refer to as a mutation [cf. Definition 3.3, (i)]. Given a diagram of mutations, one may then define the notion of a "mutation that extracts, from the diagram, a certain portion of the types of mathematical objects that appear in the diagram that is invariant with respect to the mutations in the diagram"; we refer to such a mutation as a core [cf. Definition 3.3, (v)].

One fundamental example, in the context of the present series of papers, of a diagram of mutations is the usual set-up of [absolute] anabelian geometry [cf. Example 3.5 for more details]. That is to say, one begins with the species constituted by schemes satisfying certain conditions. One then considers the mutation

$$
X \rightsquigarrow \Pi_{X}
$$

that associates to such a scheme $X$ its étale fundamental group $\Pi_{X}$ [say, considered up to inner automorphisms]. Here, it is important to note that the codomain of this mutation is the species constituted by topological groups [say, considered up to inner automorphisms] that satisfy certain conditions which do not include any information concerning how the group is related [for instance, via some sort of étale fundamental group mutation] to a scheme. The notion of an anabelian reconstruction algorithm may then be formalized as a mutation that forms a "mutation-quasi-inverse" to the fundamental group mutation.

Another fundamental example, in the context of the present series of papers, of a diagram of mutations arises from the Frobenius morphism in positive characteristic scheme theory [cf. Example 3.6 for more details]. That is to say, one fixes a prime number $p$ and considers the species constituted by reduced schemes of characteristic $p$. One then considers the mutation that associates

$$
S \rightsquigarrow S^{(p)}
$$

to such a scheme $S$ the scheme $S^{(p)}$ with the same topological space, but whose regular functions are given by the $p$-th powers of the regular functions on the original scheme. Thus, the domain and codomain of this mutation are given by the same species. One may also consider a log scheme version of this example, which, at the level of monoids, corresponds, in essence, to assigning

$$
M \rightsquigarrow p \cdot M
$$

to a torsion-free abelian monoid $M$ the submonoid $p \cdot M \subseteq M$ determined by the image of multiplication by $p$. Returning to the case of schemes, one may then observe that the well-known constructions of the perfection and the étale site

$$
S \rightsquigarrow S^{\mathrm{pf}} ; \quad S \rightsquigarrow S_{\text {ét }}
$$

associated to a reduced scheme $S$ of characteristic $p$ give rise to cores of the diagram obtained by considering iterates of the "Frobenius mutation" just discussed. 
This last example of the Frobenius mutation and the associated core constituted by the étale site is of particular importance in the context of the present series of papers in that it forms the "intuitive prototype" that underlies the theory of the vertical and horizontal lines of the log-theta-lattice [cf. the discussion of Remark 3.6.1, (i)]. One notable aspect of this example is the [evident!] fact that the domain and codomain of the Frobenius mutation are given by the same species. That is to say, despite the fact that in the construction of the scheme $S^{(p)}$ [cf. the notation of the preceding paragraph] from the scheme $S$, the scheme $S^{(p)}$ is "subordinate" to the scheme $S$, the domain and codomain species of the resulting Frobenius mutation coincide, hence, in particular, are on a par with one another. This sort of situation served, for the author, as a sort of model for the log- and $\Theta_{\text {LGP }}^{\times \mu}$-links of the log-theta-lattice, which may be formulated as mutations between the species constituted by the notion of a $\Theta^{ \pm \text {ell }} N F$-Hodge theater. That is to say, although in the construction of either the log- or the $\Theta_{\mathrm{LGP}}^{\times \boldsymbol{\mu}}$-link, the domain and codomain $\Theta^{ \pm \text {ell }} \mathrm{NF}-H o d g e$ theaters are by no means on a "par" with one another, the domain and codomain $\Theta^{ \pm \text {ell }} \mathrm{NF}$-Hodge theaters of the resulting $\mathfrak{l o g}-/ \Theta_{\mathrm{LGP}}^{\times \boldsymbol{\mu}}$-links are regarded as objects of the same species, hence, in particular, completely on a par with one another. This sort of "relativization" of distinct models of conventional scheme theory over $\mathbb{Z}$ via the notion of a $\Theta^{ \pm \text {ell }} \mathrm{NF}$-Hodge theater [cf. Fig. I.1 below; the discussion of "gluing together" such models of conventional scheme theory in [IUTchI], §I2] is one of the most characteristic features of the theory developed in the present series of papers and, in particular, lies [tautologically!] outside the framework of conventional scheme theory over $\mathbb{Z}$. That is to say, in the framework of conventional scheme theory over $\mathbb{Z}$, if one starts out with schemes over $\mathbb{Z}$ and constructs from them, say, by means of geometric objects such as the theta function on a Tate curve, some sort of Frobenioid that is isomorphic to a Frobenioid associated to $\mathbb{Z}$, then - unlike, for instance, the case of the Frobenius morphism in positive characteristic scheme theory -

there is no way, within the framework of conventional scheme theory, to treat the newly constructed Frobenioid "as if it is the Frobenioid associated to $\mathbb{Z}$, relative to some new version/model of conventional scheme theory".

\begin{tabular}{|c|c|c|c|c|}
\hline $\begin{array}{c}\text { non- } \\
\text { scheme- }\end{array}$ & $\begin{array}{c}\text { one } \\
\text { model of } \\
\text { conven- } \\
\text { tional }\end{array}$ & $\begin{array}{l}\text { non- } \\
\text { scheme- }\end{array}$ & $\begin{array}{l}\text { another } \\
\text { model of } \\
\text { conven- } \\
\text { tional }\end{array}$ & $\begin{array}{c}\text { non- } \\
\text { scheme- }\end{array}$ \\
\hline $\begin{array}{l}\text { theoretic } \\
\text { link }\end{array}$ & $\begin{array}{l}\text { scheme } \\
\text { theory } \\
\text { over } \mathbb{Z}\end{array}$ & $\begin{array}{l}\text { theoretic } \\
\text { link }\end{array}$ & $\begin{array}{l}\text { scheme } \\
\text { theory } \\
\text { over } \mathbb{Z}\end{array}$ & $\begin{array}{c}\text { theoretic } \\
\text { link }\end{array}$ \\
\hline
\end{tabular}

Fig. I.1: Relativized models of conventional scheme theory over $\mathbb{Z}$

If, moreover, one thinks of $\mathbb{Z}$ as being constructed, in the usual way, via $a x$ iomatic set theory, then one may interpret the "absolute" — i.e., "tautologically 
unrelativizable" - nature of conventional scheme theory over $\mathbb{Z}$ at a purely settheoretic level. Indeed, from the point of view of the " $\in$-structure" of axiomatic set theory, there is no way to treat sets constructed at distinct levels of this $\in$-structure as being on a par with one another. On the other hand, if one focuses not on the level of the $\in$-structure to which a set belongs, but rather on species, then the notion of a species allows one to relate - i.e., to treat on a par with one another objects belonging to the species that arise from sets constructed at distinct levels of the $\in$-structure. That is to say,

the notion of a species allows one to "simulate $\in$-loops" without violating the axiom of foundation of axiomatic set theory

— cf. the discussion of Remark 3.3.1, (i).

As one constructs sets at higher and higher levels of the $\epsilon$-structure of some model of axiomatic set theory - e.g., as one travels along vertical or horizontal lines of the log-theta-lattice! - one typically encounters new schemes, which give rise to new Galois categories, hence to new Galois or étale fundamental groups, which may only be constructed if one allows oneself to consider new basepoints, relative to new universes. In particular, one must continue to extend the universe, i.e., to modify the model of set theory, relative to which one works. Here, we recall in passing that such "extensions of universe" are possible on account of an existence axiom concerning universes, which is apparently attributed to the "Grothendieck school" and, moreover, cannot, apparently, be obtained as a consequence of the conventional ZFC axioms of axiomatic set theory [cf. the discussion at the beginning of $\S 3$ for more details]. On the other hand, ultimately in the present series of papers [cf. the discussion of [IUTchIII], Introduction], we wish to obtain algorithms for constructing various objects that arise in the context of the new schemes/universes discussed above - i.e., at distant $\Theta^{ \pm e l l} N F$-Hodge theaters of the log-theta-lattice - that make sense from the point of view the original schemes/universes that occurred at the outset of the discussion. Again, the fundamental tool that makes this possible, i.e., that allows one to express constructions in the new universes in terms that makes sense in the original universe is precisely

the species-theoretic formulation - i.e., the formulation via settheoretic formulas that do not depend on particular choices invoked in particular universes - of the constructions of interest

- cf. the discussion of Remarks 3.1.2, 3.1.3, 3.1.4, 3.1.5, 3.6.2, 3.6.3. This is the point of view that gave rise to the term "inter-universal". At a more concrete level, this "inter-universal" contact between constructions in distant models of conventional scheme theory in the log-theta-lattice is realized by considering [the étale-like structures given by] the various Galois or étale fundamental groups that occur as [the "type of mathematical object", i.e., species constituted by] abstract topological groups [cf. the discussion of Remark 3.6.3, (i); [IUTchI], §I3]. These abstract topological groups give rise to vertical or horizontal cores of the log-theta-lattice. Moreover, once one obtains cores that are sufficiently "nondegenerate", or "rich in structure", so as to serve as containers for the non-coric portions of the various mutations [e.g., vertical and horizontal arrows of the logtheta-lattice] under consideration, then one may construct the desired algorithms, 
or descriptions, of these non-coric portions in terms of coric containers, up to certain relatively mild indeterminacies [i.e., which reflect the non-coric nature of these non-coric portions!] — cf. the illustration of this sort of situation given in Fig. I.2 below; Remark 3.3.1, (iii); Remark 3.6.1, (ii). In the context of the logtheta-lattice, this is precisely the sort of situation that was achieved in [IUTchIII], Theorem A [cf. the discussion of [IUTchIII], Introduction].
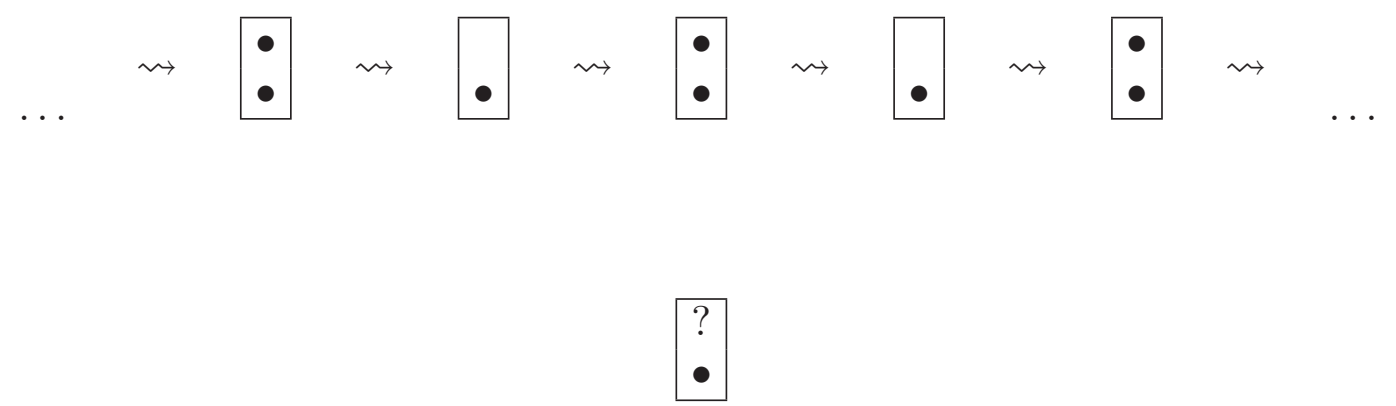

Fig. I.2: A coric container underlying a sequence of mutations

In the context of the above discussion of set-theoretic aspects of the theory developed in the present series of papers, it is of interest to note the following observation, relative to the analogy between the theory of the present series of papers and $\boldsymbol{p}$-adic Teichmüller theory [cf. the discussion of [IUTchI], §I4]. If, instead of working species-theoretically, one attempts to document all of the possible choices that occur in various newly introduced universes that occur in a construction, then one finds that one is obliged to work with sets, such as sets obtained via set-theoretic exponentiation, of very large cardinality. Such sets of large cardinality are reminiscent of the exponentially large denominators that occur if one attempts to $p$-adically formally integrate an arbitrary connection as opposed to a canonical crystalline connection of the sort that occurs in the context of the canonical liftings of $p$-adic Teichmüller theory [cf. the discussion of Remark 3.6.2, (iii)]. In this context, it is of interest to recall the computations of [Finot], which assert, roughly speaking, that the canonical liftings of $p$-adic Teichmüller theory may, in certain cases, be characterized as liftings "of minimal complexity" in the sense that their Witt vector coordinates are given by polynomials of minimal degree.

Finally, we observe that although, in the above discussion, we concentrated on the similarities, from an "inter-universal" point of view, between the vertical and horizontal arrows of the log-theta-lattice, there is one important difference between these vertical and horizontal arrows: namely,

- whereas the copies of the full arithmetic fundamental group - i.e., in particular, the copies of the geometric fundamental group - on either side of a vertical arrow are identified with one another,

- in the case of a horizontal arrow, only the Galois groups of the local base fields on either side of the arrow are identified with one another

- cf. the discussion of Remark 3.6.3, (ii). One way to understand the reason for this difference is as follows. In the case of the vertical arrows - i.e., the loglinks, which, in essence, amount to the various local p-adic logarithms - in order 
to construct the log-link, it is necessary to make use, in an essential way, of the local ring structures at $\underline{v} \in \underline{\mathbb{V}}$ [cf. the discussion of [IUTchIII], Definition 1.1, (i), (ii)], which may only be reconstructed from the full arithmetic fundamental group. By contrast, in order to construct the horizontal arrows - i.e., the $\Theta_{\text {LGP }^{-}}^{\times \mu}$ links - this local ring structure is unnecessary. On the other hand, in order to construct the horizontal arrows, it is necessary to work with structures that, up to isomorphism, are common to both the domain and the codomain of the arrow. Since the construction of the domain of the $\Theta_{\text {LGP }}^{\times \mu}$-link depends, in an essential way, on the Gaussian monoids, i.e., on the labels $\in \mathbb{F}_{l}^{*}$ for the theta values, which are constructed from the geometric fundamental group, while the codomain

only involves monoids arising from the local q-parameters " $\underline{\underline{q}}$ " $\left[\right.$ for $\left.\underline{v} \in \underline{\mathbb{V}}^{\text {bad }}\right]$, which are constructed in a fashion that is independent of these labels, in order to obtain an isomorphism between structures arising from the domain and codomain, it is necessary to restrict one's attention to the Galois groups of the local base fields, which are free of any dependence on these labels.

\section{Acknowledgements:}

I would like to thank Fumiharu Kato and Akio Tamagawa for many helpful discussions concerning the material presented in this paper. Also, I would like to thank Kentaro Sato for informing me of [Ffmn].

\section{Notations and Conventions:}

We shall continue to use the "Notations and Conventions" of [IUTchI], $\S 0$. 


\section{Section 1: Log-volume Estimates}

In the present $\S 1$, we perform various elementary local computations concerning nonarchimedean and archimedean local fields which allow us to obtain more explicit versions [cf. Theorem 1.10 below] of the log-volume estimates for $\Theta$ pilot objects obtained in [IUTchIII], Corollary 3.12 .

In the following, if $\lambda \in \mathbb{R}$, then we shall write

$\lceil\lambda\rceil$ (respectively, $\lfloor\lambda\rfloor$ )

for the smallest (respectively, largest) $n \in \mathbb{Z}$ such that $n \geq \lambda$ (respectively, $n \leq \lambda$ ).

Proposition 1.1. (Multiple Tensor Products and Differents) Let $p$ be a prime number, I a nonempty finite set, $\overline{\mathbb{Q}}_{p}$ an algebraic closure of $\mathbb{Q}_{p}$. Write $\bar{R} \subseteq \overline{\mathbb{Q}}_{p}$ for the ring of integers of $\overline{\mathbb{Q}}_{p}$ and ord : $\overline{\mathbb{Q}}_{p}^{\times} \rightarrow \mathbb{Q}$ for the natural $p$-adic valuation on $\overline{\mathbb{Q}}_{p}$, normalized so that ord $(p)=1$. For $i \in I$, let $k_{i} \subseteq \overline{\mathbb{Q}}_{p}$ be a finite extension of $\mathbb{Q}_{p}$; write $R_{i} \stackrel{\text { def }}{=} \mathcal{O}_{k_{i}}=\bar{R} \cap k_{i}$ for the ring of integers of $k_{i}$ and $\mathfrak{d}_{i} \in \mathbb{Q} \geq 0$ for the order [i.e., "ord $(-)$ "] of any generator of the different ideal of $R_{i}$ over $\mathbb{Z}_{p}$. Also, for any nonempty subset $E \subseteq I$, let us write

$$
R_{E} \stackrel{\text { def }}{=} \bigotimes_{i \in E} R_{i} ; \quad \mathfrak{d}_{E} \stackrel{\text { def }}{=} \sum_{i \in E} \mathfrak{d}_{i}
$$

- where the tensor product is over $\mathbb{Z}_{p}$. Fix an element $* \in I ;$ write $I^{*} \stackrel{\text { def }}{=} I \backslash\{*\}$. Then

$$
R_{I} \subseteq\left(R_{I}\right)^{\sim} ; \quad p^{\left\lceil\mathfrak{d}_{I^{*}}\right\rceil} \cdot\left(R_{I}\right)^{\sim} \subseteq R_{I}
$$

- where we write "(-) " for the normalization of the ring in parentheses.

Proof. Let us regard $R_{I}$ as an $R_{*}$-algebra in the evident fashion. It is immediate from the definitions that $R_{I} \subseteq\left(R_{I}\right)^{\sim}$. Now observe that

$$
\bar{R} \otimes_{R_{*}} R_{I} \subseteq \bar{R} \otimes_{R_{*}}\left(R_{I}\right)^{\sim} \subseteq\left(\bar{R} \otimes_{R_{*}} R_{I}\right)^{\sim}
$$

— where $\left(\bar{R} \otimes_{R_{*}} R_{I}\right)^{\sim}$ decomposes as a direct sum of finitely many copies of $\bar{R}$. In particular, one verifies immediately, in light of the fact the $\bar{R}$ is faithfully flat over $R_{*}$, that to complete the proof of Proposition 1.1, it suffices to verify that

$$
p^{\left\lceil\mathfrak{d}_{I^{*}}\right\rceil} \cdot\left(\bar{R} \otimes_{R_{*}} R_{I}\right)^{\sim} \subseteq \bar{R} \otimes_{R_{*}} R_{I}
$$

— or, indeed, that

$$
p^{\mathfrak{d}_{I} I^{*}} \cdot\left(\bar{R} \otimes_{R_{*}} R_{I}\right)^{\sim} \subseteq \bar{R} \otimes_{R_{*}} R_{I}
$$

— where, for $\lambda \in \mathbb{Q}$, we write $p^{\lambda}$ for any element of $\overline{\mathbb{Q}}_{p}$ such that $\operatorname{ord}\left(p^{\lambda}\right)=\lambda$. On other hand, it follows immediately from induction on the cardinality of $I$ that 
to verify this last inclusion, it suffices to verify the inclusion in the case where $I$ is of cardinality two. But in this case, the desired inclusion follows immediately from the definition of the different ideal. This completes the proof of Proposition 1.1.

Proposition 1.2. (Differents and Logarithms) We continue to use the notation of Proposition 1.1. For $i \in I$, write $e_{i}$ for the ramification index of $k_{i}$ over $\mathbb{Q}_{p}$

$$
a_{i} \stackrel{\text { def }}{=} \frac{1}{e_{i}} \cdot\left\lceil\frac{e_{i}}{p-2}\right\rceil \text { if } p>2, \quad a_{i} \stackrel{\text { def }}{=} 2 \text { if } p=2 .
$$

Here, $" \log (-) "$ denotes the natural logarithm. Thus,

$$
\text { if } p>2 \text { and } e_{i} \leq p-2 \text {, then } a_{i}=\frac{1}{e_{i}}
$$

For any nonempty subset $E \subseteq I$, let us write

$$
\log _{p}\left(R_{E}^{\times}\right) \stackrel{\text { def }}{=} \bigotimes_{i \in E} \log _{p}\left(R_{i}^{\times}\right) ; \quad a_{E} \stackrel{\text { def }}{=} \sum_{i \in E} a_{i}
$$

- where the tensor product is over $\mathbb{Z}_{p}$; we write " $\log _{p}(-)$ " for the $p$-adic logarithm. For $\lambda \in \frac{1}{e_{i}} \cdot \mathbb{Z}$, we shall write $p^{\lambda} \cdot R_{i}$ for the fractional ideal of $R_{i}$ generated by any element " $p^{\lambda}$ " of $k_{i}$ such that $\operatorname{ord}\left(p^{\lambda}\right)=\lambda$. Let

$$
\phi: \log _{p}\left(R_{I}^{\times}\right) \otimes \mathbb{Q}_{p} \stackrel{\sim}{\rightarrow} \log _{p}\left(R_{I}^{\times}\right) \otimes \mathbb{Q}_{p}
$$

be an automorphism of the finite dimensional $\mathbb{Q}_{p}$-vector space $\log _{p}\left(R_{I}^{\times}\right) \otimes \mathbb{Q}_{p}$ that induces an automorphism of the submodule $\log _{p}\left(R_{I}^{\times}\right)$. Then:

(i) We have:

$$
p^{a_{i}} \cdot R_{i} \subseteq \log _{p}\left(R_{i}^{\times}\right)
$$

- where the "ᄃ" is an equality when $p>2$ and $e_{i} \leq p-2$.

(ii) We have:

$$
\phi\left(p^{\lambda} \cdot R_{i} \otimes_{R_{i}}\left(R_{I}\right)^{\sim}\right) \subseteq p^{\lfloor\lambda\rfloor-\left\lceil\mathfrak{d}_{I}\right\rceil-\left\lceil a_{I}\right\rceil} \cdot \log _{p}\left(R_{I}^{\times}\right)
$$

for any $\lambda \in \frac{1}{e_{i}} \cdot \mathbb{Z}, i \in I$. In particular, $\phi\left(\left(R_{I}\right)^{\sim}\right) \subseteq p^{-\left\lceil\mathfrak{o}_{I}\right\rceil-\left\lceil a_{I}\right\rceil} \cdot \log _{p}\left(R_{I}^{\times}\right)$.

(iii) Suppose that $p>2$, and that $e_{i} \leq p-2$ for all $i \in I$. Then we have:

$$
\phi\left(p^{\lambda} \cdot R_{i} \otimes_{R_{i}}\left(R_{I}\right)^{\sim}\right) \subseteq p^{\lfloor\lambda\rfloor-\left\lceil\mathfrak{d}_{I}\right\rceil-1} \cdot\left(R_{I}\right)^{\sim}
$$

for any $\lambda \in \frac{1}{e_{i}} \cdot \mathbb{Z}, i \in I$. In particular, $\phi\left(\left(R_{I}\right)^{\sim}\right) \subseteq p^{-\left\lceil\mathfrak{d}_{I}\right\rceil-1} \cdot\left(R_{I}\right)^{\sim}$.

(iv) If $p>2$ and $e_{i}=1$ for all $i \in I$, then $\phi\left(\left(R_{I}\right)^{\sim}\right) \subseteq\left(R_{I}\right)^{\sim}$.

Proof. Assertion (i) follows immediately from the well-known theory of the p-adic logarithm and exponential maps [cf., e.g., [Kobl], p. 81]. Next, let us observe that 
to verify assertions (ii) and (iii), it suffices to consider the case where $\lambda=0$. Now it follows from the second displayed inclusion of Proposition 1.1 that

$$
p^{\left\lceil\mathfrak{o}_{I}\right\rceil} \cdot\left(R_{I}\right)^{\sim} \subseteq R_{I}=\bigotimes_{i \in I} R_{i}
$$

and hence that

$$
p^{\left\lceil\mathfrak{d}_{I}\right\rceil+\left\lceil a_{I}\right\rceil} \cdot\left(R_{I}\right)^{\sim} \subseteq \bigotimes_{i \in I} p^{a_{i}} \cdot R_{i} \subseteq \bigotimes_{i \in I} \log _{p}\left(R_{i}^{\times}\right)=\log _{p}\left(R_{I}^{\times}\right)
$$

- where the first inclusion follows immediately from the fact that $\left(R_{I}\right)^{\sim}$ decomposes as a direct sum of rings of integers of finite extensions of $\mathbb{Q}_{p}$, and the second inclusion follows from assertion (i). Thus, assertion (ii) follows immediately from the fact that $\phi$ induces an automorphism of the submodule $\log _{p}\left(R_{I}^{\times}\right)$. When $p>2$ and $e_{i} \leq p-2$ for all $i \in I$, we thus obtain that

$$
p^{\left\lceil\mathfrak{d}_{I}\right\rceil+\left\lceil a_{I}\right\rceil} \cdot \phi\left(\left(R_{I}\right)^{\sim}\right) \subseteq \log _{p}\left(R_{I}^{\times}\right)=\bigotimes_{i \in I} p^{a_{i}} \cdot R_{i} \subseteq p^{\left\lfloor a_{I}\right\rfloor} \cdot\left(R_{I}\right)^{\sim}
$$

- where the equality follows from assertion (i), and the final inclusion follows immediately from the fact that $\left(R_{I}\right)^{\sim}$ decomposes as a direct sum of rings of integers of finite extensions of $\mathbb{Q}_{p}$. Thus, assertions (iii) and (iv) follow immediately from the fact that $\left\lfloor a_{I}\right\rfloor-\left\lceil a_{I}\right\rceil \geq-1$, together with the fact that $a_{i}=1, \mathfrak{d}_{i}=0$ whenever $e_{i}=1$. This completes the proof of Proposition 1.2.

Proposition 1.3. (Estimates of Differents) We continue to use the notation of Proposition 1.2. Suppose that $k_{0} \subseteq k_{i}$ is a subfield that contains $\mathbb{Q}_{p}$. Write $R_{0} \stackrel{\text { def }}{=} \mathcal{O}_{k_{0}}$ for the ring of integers of $k_{0}, \mathfrak{d}_{0}$ for the order [i.e., "ord(-)"] of any generator of the different ideal of $R_{0}$ over $\mathbb{Z}_{p}$, $e_{0}$ for the ramification index of $k_{0}$ over $\mathbb{Q}_{p}, e_{i / 0} \stackrel{\text { def }}{=} e_{i} / e_{0}(\in \mathbb{Z}),\left[k_{i}: k_{0}\right]$ for the degree of the extension $k_{i} / k_{0}, n_{i}$ for the unique nonnegative integer such that $\left[k_{i}: k_{0}\right] / p^{n_{i}}$ is an integer prime to $p$. Then:

(i) We have:

$$
\mathfrak{d}_{i} \geq \mathfrak{d}_{0}+\left(e_{i / 0}-1\right) /\left(e_{i / 0} \cdot e_{0}\right)=\mathfrak{d}_{0}+\left(e_{i / 0}-1\right) / e_{i}
$$

- where the " $\geq$ " is an equality when $k_{i}$ is tamely ramified over $k_{0}$.

(ii) Suppose that $k_{i}$ is a finite Galois extension of a subfield $k_{1} \subseteq k_{i}$ such that $k_{0} \subseteq k_{1}$, and $k_{1}$ is tamely ramified over $k_{0}$. Then we have: $\mathfrak{d}_{i} \leq \mathfrak{d}_{0}+n_{i}+1 / e_{0}$.

Proof. First, we consider assertion (i). By replacing $k_{0}$ by an unramified extension of $k_{0}$ contained in $k_{i}$, we may assume without loss of generality that $k_{i}$ is a totally ramified extension of $k_{0}$. Let $\pi_{0}$ be a uniformizer of $R_{0}$. Then there exists an isomorphism $R_{0}$-algebras $R_{0}[x] /(f(x)) \stackrel{\sim}{\rightarrow} R_{i}$, where $f(x) \in R_{0}[x]$ is a monic 
polynomial which is $\equiv x^{e_{i / 0}}\left(\bmod \pi_{0}\right)$, that maps $x \mapsto \pi_{i}$ for some uniformizer $\pi_{i}$ of $R_{i}$. Thus, the different $\mathfrak{d}_{i}$ may be computed as follows:

$$
\begin{aligned}
\mathfrak{d}_{i}-\mathfrak{d}_{0} & =\operatorname{ord}\left(f^{\prime}\left(\pi_{i}\right)\right) \geq \min \left(\operatorname{ord}\left(\pi_{0}\right), \operatorname{ord}\left(e_{i / 0} \cdot \pi_{i}^{e_{i / 0}-1}\right)\right) \\
& \geq \min \left(\frac{1}{e_{0}}, \operatorname{ord}\left(\pi_{i}^{e_{i / 0}-1}\right)\right)=\min \left(\frac{1}{e_{0}}, \frac{e_{i / 0}-1}{e_{i / 0} \cdot e_{0}}\right)=\frac{e_{i / 0}-1}{e_{i}}
\end{aligned}
$$

— where, for $\lambda, \mu \in \mathbb{R}$ such that $\lambda \geq \mu$, we define $\min (\lambda, \mu) \stackrel{\text { def }}{=} \mu$. When $k_{i}$ is tamely ramified over $k_{0}$, one verifies immediately that the inequalities of the above display are, in fact, equalities. This completes the proof of assertion (i).

Next, we consider assertion (ii). We apply induction on $n_{i}$. Since assertion (ii) follows immediately from assertion (i) when $n_{i}=0$, we may assume that $n_{i} \geq 1$, and that assertion (ii) has been verified for smaller " $n_{i}$ ". By replacing $k_{1}$ by some tamely ramified extension of $k_{1}$ contained in $k_{i}$, we may assume without loss of generality that $\operatorname{Gal}\left(k_{i} / k_{1}\right)$ is a $p$-group. Since $p$-groups are solvable, it follows that there exists a subextension $k_{1} \subseteq k_{*} \subseteq k_{i}$ such that $k_{i} / k_{*}$ and $k_{*} / k_{1}$ are Galois extensions of degree $p$ and $p^{n_{i}-1}$, respectively. Write $R_{*} \stackrel{\text { def }}{=} \mathcal{O}_{k_{*}}$ for the ring of integers of $k_{*}, \mathfrak{d}_{*}$ for the order [i.e., "ord(-)"] of any generator of the different ideal of $R_{*}$ over $\mathbb{Z}_{p}$, and $e_{*}$ for the ramification index of $k_{*}$ over $\mathbb{Q}_{p}$. Thus, by the induction hypothesis, it follows that $\mathfrak{d}_{*} \leq \mathfrak{d}_{0}+n_{i}-1+1 / e_{0}$. To verify that $\mathfrak{d}_{i} \leq \mathfrak{d}_{0}+n_{i}+1 / e_{0}$, it suffices to verify that $\mathfrak{d}_{i} \leq \mathfrak{d}_{0}+n_{i}+1 / e_{0}+\epsilon$ for any positive real number $\epsilon$. By possibly enlarging $k_{i}$ and $k_{1}$, we may also assume without loss of generality that the tamely ramified extension $k_{1}$ of $k_{0}$ contains a primitive $p$-th root of unity, and, moreover, that the ramification index $e_{1}$ of $k_{1}$ over $\mathbb{Q}_{p}$ satisfies $e_{1} \geq p / \epsilon\left[\right.$ so $\left.e_{*} \geq e_{1} \geq p / \epsilon\right]$. Thus, $k_{i}$ is a Kummer extension of $k_{*}$. In particular, there exists an inclusion of $R_{*}$-algebras $R_{*}[x] /(f(x)) \hookrightarrow R_{i}$, where $f(x) \in R_{*}[x]$ is a monic polynomial which is of the form $f(x)=x^{p}-\varpi_{*}$ for some element $\varpi_{*}$ of $R_{*}$ satisfying $0 \leq \operatorname{ord}\left(\varpi_{*}\right) \leq \frac{p-1}{e_{*}}$, that maps $x \mapsto \varpi_{i}$ for some element $\varpi_{i}$ of $R_{i}$ satisfying $0 \leq \operatorname{ord}\left(\varpi_{i}\right) \leq \frac{p-1}{p \cdot e_{*}}$. Now we compute:

$$
\begin{aligned}
\mathfrak{d}_{i} & \leq \operatorname{ord}\left(f^{\prime}\left(\varpi_{i}\right)\right)+\mathfrak{d}_{*} \leq \operatorname{ord}\left(p \cdot \varpi_{i}^{p-1}\right)+\mathfrak{d}_{0}+n_{i}-1+1 / e_{0} \\
& =(p-1) \cdot \operatorname{ord}\left(\varpi_{i}\right)+\mathfrak{d}_{0}+n_{i}+1 / e_{0} \leq \frac{(p-1)^{2}}{p \cdot e_{*}}+\mathfrak{d}_{0}+n_{i}+1 / e_{0} \\
& \leq \frac{p}{e_{*}}+\mathfrak{d}_{0}+n_{i}+1 / e_{0} \leq \mathfrak{d}_{0}+n_{i}+1 / e_{0}+\epsilon
\end{aligned}
$$

— thus completing the proof of assertion (ii).

\section{Proposition 1.4. (Nonarchimedean Normalized Log-volume Estimates)} We continue to use the notation of Proposition 1.2. Also, for $i \in I$, write $R_{i}^{\mu} \subseteq R_{i}^{\times}$ for the torsion subgroup of $R_{i}^{\times}, R_{i}^{\times \mu} \stackrel{\text { def }}{=} R_{i}^{\times} / R_{i}^{\mu}, p^{f_{i}}$ for the cardinality of the residue field of $k_{i}$, and $p^{m_{i}}$ for the order of the p-primary component of $R_{i}^{\mu}$. Thus, the order of $R_{i}^{\mu}$ is equal to $p^{m_{i}} \cdot\left(p^{f_{i}}-1\right)$. Then:

(i) The log-volumes constructed in [AbsTopIII], Proposition 5.7, (i), on the various finite extensions of $\mathbb{Q}_{p}$ contained in $\overline{\mathbb{Q}}_{p}$ may be suitably normalized [i.e., by dividing by the degree of the finite extension] so as to yield a notion of log-volume

$$
\mu^{\log }(-)
$$


defined on compact open subsets of finite extensions of $\mathbb{Q}_{p}$ contained in $\overline{\mathbb{Q}}_{p}$, valued in $\mathbb{R}$, and normalized so that $\mu^{\log }\left(R_{i}\right)=0, \mu^{\log }\left(p \cdot R_{i}\right)=-\log (p)$, for each $i \in I$. Moreover, by applying the fact that tensor products of finitely many copies of $\overline{\mathbb{Q}}_{p}$ over $\mathbb{Z}_{p}$ decompose, naturally, as direct sums of finitely many copies of $\overline{\mathbb{Q}}_{p}$, we obtain a notion of log-volume - which, by abuse of notation, we shall also denote by " $\mu^{\log }(-) "$ - defined on compact open subsets of finitely generated $\mathbb{Z}_{p}$-submodules of such tensor products, valued in $\mathbb{R}$, and normalized so that $\mu^{\log }\left(\left(R_{E}\right)^{\sim}\right)=0$, $\mu^{\log }\left(p \cdot\left(R_{E}\right)^{\sim}\right)=-\log (p)$, for any nonempty set $E \subseteq I$.

(ii) We have:

$$
\mu^{\log }\left(\log _{p}\left(R_{i}^{\times}\right)\right)=-\left(\frac{1}{e_{i}}+\frac{m_{i}}{e_{i} f_{i}}\right) \cdot \log (p)
$$

[cf. [AbsTopIII], Proposition 5.8, (iii)].

(iii) Let $I^{*} \subseteq I$ be a subset such that for each $i \in I \backslash I^{*}$, it holds that $p-2 \geq$ $e_{i}(\geq 1)$. Then for any $\lambda \in \frac{1}{e_{i \dagger}} \cdot \mathbb{Z}, i^{\dagger} \in I$, we have $\phi\left(p^{\lambda} \cdot R_{i^{\dagger}} \otimes_{R_{i \dagger}}\left(R_{I}\right)^{\sim}\right) \subseteq$ $p^{\lfloor\lambda\rfloor-\left\lceil\mathfrak{d}_{I}\right\rceil-\left\lceil a_{I}\right\rceil} \cdot \log _{p}\left(R_{I}^{\times}\right)$, and

$$
\mu^{\log }\left(p^{\lfloor\lambda\rfloor-\left\lceil\mathfrak{d}_{I}\right\rceil-\left\lceil a_{I}\right\rceil} \cdot \log _{p}\left(R_{I}^{\times}\right)\right) \leq\left(-\lambda+\mathfrak{d}_{I}+3+4 \cdot\left|I^{*}\right| / p\right) \cdot \log (p)
$$

- where we write $\left|I^{*}\right|$ for the cardinality of $I^{*}$. Moreover, $\left\lceil\mathfrak{d}_{I}\right\rceil+\left\lceil a_{I}\right\rceil \geq|I|$ if $p>2 ;\left\lceil\mathfrak{d}_{I}\right\rceil+\left\lceil a_{I}\right\rceil \geq 2 \cdot|I|$ if $p=2$.

(iv) If $p>2$ and $e_{i}=1$ for all $i \in I$, then $\phi\left(\left(R_{I}\right)^{\sim}\right) \subseteq\left(R_{I}\right)^{\sim}$, and $\mu^{\log }\left(\left(R_{I}\right)^{\sim}\right)=0$.

Proof. Assertion (i) follows immediately from the definitions. Next, we consider assertion (ii). Note that the log-volume on $R_{i}^{\times}$determines, in a natural way, a log-volume on the quotient $R_{i}^{\times} \rightarrow R_{i}^{\times \mu}$. Moreover, in light of the compatibility of the $\log$-volume with " $\log _{p}(-)$ " [cf. [AbsTopIII], Proposition 5.7, (i), (c)], it follows immediately that $\mu^{\log }\left(\log _{p}\left(R_{i}^{\times}\right)\right)=\mu^{\log }\left(R_{i}^{\times \mu}\right)$. Thus, it suffices to compute $e_{i} \cdot f_{i} \cdot \mu^{\log }\left(R_{i}^{\times \mu}\right)=e_{i} \cdot f_{i} \cdot \mu^{\log }\left(R_{i}^{\times}\right)-\log \left(p^{m_{i}} \cdot\left(p^{f_{i}}-1\right)\right)$. On the other hand, it follows immediately from the basic properties of the log-volume [cf. [AbsTopIII], Proposition 5.7, (i), (a)] that $e_{i} \cdot f_{i} \cdot \mu^{\log }\left(R_{i}^{\times}\right)=\log \left(1-p^{-f_{i}}\right)$, so $e_{i} \cdot f_{i} \cdot \mu^{\log }\left(R_{i}^{\times \boldsymbol{\mu}}\right)=$ $-\left(f_{i}+m_{i}\right) \cdot \log (p)$, as desired. This completes the proof of assertion (ii).

The inclusion of assertion (iii) follows immediately from Proposition 1.2, (ii). When $p=2$, the fact that $\left\lceil\mathfrak{d}_{I}\right\rceil+\left\lceil a_{I}\right\rceil \geq 2 \cdot|I|$ follows immediately from the definition of " $a_{i}$ " in Proposition 1.2. When $p>2$, it follows immediately from the definition of " $a_{i}$ " in Proposition 1.2 that $a_{i} \geq 1 / e_{i}$, for all $i \in I$; thus, since $\mathfrak{d}_{i} \geq\left(e_{i}-1\right) / e_{i}$ for all $i \in I$ [cf. Proposition 1.3, (i)], we conclude that $\mathfrak{d}_{i}+a_{i} \geq 1$ for all $i \in I$, and hence that $\left\lceil\mathfrak{d}_{I}\right\rceil+\left\lceil a_{I}\right\rceil \geq \mathfrak{d}_{I}+a_{I} \geq|I|$, as asserted in the stament of assertion (iii). Next, let us observe that $\frac{1}{p-2} \leq \frac{4}{p}$ for $p \geq 3$. Thus, it follows immediately from the definition of $a_{i}$ in Proposition 1.2 that $a_{i} \leq \frac{4}{p}+\frac{1}{e_{i}}$ for $i \in I, a_{i}=\frac{1}{e_{i}}$ for $i \in I \backslash I^{*}$. On the other hand, by assertion (i), we have 
$\mu^{\log }\left(R_{I}\right) \leq \mu^{\log }\left(\left(R_{I}\right)^{\sim}\right)=0$; by assertion (ii), we have $\mu^{\log }\left(\log _{p}\left(R_{i}^{\times}\right)\right) \leq-\frac{1}{e_{i}} \cdot \log (p)$. Now we compute:

$$
\begin{aligned}
\mu^{\log }\left(p^{\lfloor\lambda\rfloor-\left\lceil\mathfrak{d}_{I}\right\rceil-\left\lceil a_{I}\right\rceil} \cdot \log _{p}\left(R_{I}^{\times}\right)\right) \leq & \left(-\lambda+\mathfrak{d}_{I}+a_{I}+3\right) \cdot \log (p)+\mu^{\log }\left(\log _{p}\left(R_{I}^{\times}\right)\right) \\
\leq & \left(-\lambda+\mathfrak{d}_{I}+a_{I}+3\right) \cdot \log (p) \\
& +\left\{\sum_{i \in I} \mu^{\log }\left(\log _{p}\left(R_{i}^{\times}\right)\right)\right\}+\mu^{\log }\left(R_{I}\right) \\
\leq & \left\{-\lambda+\mathfrak{d}_{I}+3+\sum_{i \in I}\left(a_{i}-\frac{1}{e_{i}}\right)\right\} \cdot \log (p) \\
\leq & \left(-\lambda+\mathfrak{d}_{I}+3+4 \cdot\left|I^{*}\right| / p\right) \cdot \log (p)
\end{aligned}
$$

— thus completing the proof of assertion (iii). Assertion (iv) follows immediately from assertion (i) and Proposition 1.2, (iv).

Proposition 1.5. (Archimedean Metric Estimates) In the following, we shall regard the complex archimedean field $\mathbb{C}$ as being equipped its standard Hermitian metric, i.e., the metric determined by the complex norm. Let us refer to as the primitive automorphisms of $\mathbb{C}$ the group of automorphisms lof order 8] of the underlying metrized real vector space of $\mathbb{C}$ generated by the operations of complex conjugation and multiplication by \pm 1 or $\pm \sqrt{-1}$.

(i) (Direct Sum vs. Tensor Product Metrics) The metric on $\mathbb{C}$ determines a tensor product metric on $\mathbb{C} \otimes_{\mathbb{R}} \mathbb{C}$, as well as a direct sum metric on $\mathbb{C} \oplus \mathbb{C}$. Then, relative to these metrics, any isomorphism of topological rings [i.e., arising from the Chinese remainder theorem]

$$
\mathbb{C} \otimes_{\mathbb{R}} \mathbb{C} \stackrel{\sim}{\rightarrow} \mathbb{C} \oplus \mathbb{C}
$$

is compatible with these metrics, up a factor of 2 , i.e., the metric on the righthand side corresponds to 2 times the metric on the left-hand side. [Thus, lengths differ by a factor of $\sqrt{2}$.]

(ii) (Direct Sum vs. Tensor Product Automorphisms) Relative to the notation of $(i)$, the direct sum decomposition $\mathbb{C} \oplus \mathbb{C}$, together with its Hermitian metric, is preserved, relative to the displayed isomorphism of (i), by the automorphisms of $\mathbb{C} \otimes_{\mathbb{R}} \mathbb{C}$ induced by the various primitive automorphisms of the two copies of "CC" that appear in the tensor product $\mathbb{C} \otimes_{\mathbb{R}} \mathbb{C}$.

(iii) (Direct Sums and Tensor Products of Multiple Copies) Let I, $V$ be nonempty finite sets, whose cardinalities we denote by $|I|,|V|$, respectively. Write

$$
M \stackrel{\text { def }}{=} \bigoplus_{v \in V} \mathbb{C}_{v}
$$

for the direct sum of copies $\mathbb{C}_{v} \stackrel{\text { def }}{=} \mathbb{C}$ of $\mathbb{C}$ labeled by $v \in V$, which we regard as equipped with the direct sum metric, and

$$
M_{I} \stackrel{\text { def }}{=} \bigotimes_{i \in I} M_{i}
$$


for the tensor product over $\mathbb{R}$ of copies $M_{i} \stackrel{\text { def }}{=} M$ of $M$ labeled by $i \in I$, which we regard as equipped with the tensor product metric [cf. the constructions of [IUTchIII], Proposition 3.2, (ii)]. Then the topological ring structure on each $\mathbb{C}_{v}$ determines a topological ring structure on $M_{I}$ with respect to which $M_{I}$ admits a unique direct sum decomposition as a direct sum of

$$
2^{|I|-1} \cdot|V|^{|I|}
$$

copies of $\mathbb{C}$ [cf. [IUTchIII], Proposition 3.1, (i)]. The direct sum metric on $M_{I}$ - i.e., the metric determined by the natural metrics on these copies of $\mathbb{C}$ - is equal to

$$
2^{|I|-1}
$$

times the original tensor product metric on $M_{I}$. Write

$$
B_{I} \subseteq M_{I}
$$

for the "integral structure" [cf. the constructions of [IUTchIII], Proposition 3.1, (ii)] given by the direct product of the unit balls of the copies of $\mathbb{C}$ that occur in the direct sum decomposition of $M_{I}$. Then the tensor product metric on $M_{I}$, the direct sum decomposition of $M_{I}$, the direct sum metric on $M_{I}$, and the integral structure $B_{I} \subseteq M_{I}$ are preserved by the automorphisms of $M_{I}$ induced by the various primitive automorphisms of the direct summands " $\mathbb{C}_{v}$ " that appear in the factors " $M_{i}$ " of the tensor product $M_{I}$.

(iv) (Tensor Product of Vectors of a Given Length) Suppose that we are in the situation of (iii). Fix $\lambda \in \mathbb{R}_{>0}$. Then

$$
M_{I} \quad \ni \bigotimes_{i \in I} m_{i} \quad \in \quad \lambda^{|I|} \cdot B_{I}
$$

for any collection of elements $\left\{m_{i} \in M_{i}\right\}_{i \in I}$ such that the component of $m_{i}$ in each direct summand " $\mathbb{C}_{v}$ " of $M_{i}$ is of length $\lambda$.

Proof. Assertions (i) and (ii) are discussed in [IUTchIII], Remark 3.9.1, (ii), and may be verified by means of routine and elementary arguments. Assertion (iii) follows immediately from assertions (i) and (ii). Assertion (iv) follows immediately from the various definitions involved.

Proposition 1.6. (The Prime Number Theorem) If $n$ is a positive integer, then let us write $p_{n}$ for the $n$-th largest prime number. [Thus, $p_{1}=2, p_{2}=3$, and so on.] Then there exists an integer $n_{0}$ such that holds that

$$
\sum_{m=1}^{n} \frac{\log \left(p_{m}\right)}{p_{m}} \leq 2 \cdot \log (n) \quad\left(\leq 2 \cdot \log \left(p_{n}\right)\right)
$$

for all $n \geq n_{0}$. In particular, there exists a positive real number $\eta_{\mathrm{prm}}$ such that

$$
\sum_{\eta \leq p^{-1}} \frac{\log (p)}{p} \leq-2 \cdot \log (\eta)
$$


- where the sum ranges over the prime numbers $p$ such that $\eta \leq p^{-1}$ - for all positive real $\eta<\eta_{\text {prm }}$.

Proof. Relative to our notation, the Prime Number Theorem [cf., e.g., [DmMn], $\S 3.10]$ implies that

$$
\lim _{m \rightarrow \infty} \frac{m \cdot \log \left(p_{m}\right)}{p_{m}}=1
$$

- i.e., in particular, that for some positive integer $m_{0}$, it holds that

$$
\frac{\log \left(p_{m}\right)}{p_{m}} \leq \frac{4}{3} \cdot \frac{1}{m}
$$

for all $m \geq m_{0}$. On the other hand, one verifies immediately [i.e., by estimating the integral of the function $\mathbb{R}_{>0} \ni x \mapsto \frac{1}{x} \in \mathbb{R}_{>0}$ ] that $m_{0}$ may be chosen so that

$$
\sum_{m=1}^{n} \frac{1}{m} \leq \frac{4}{3} \cdot \log (n)
$$

for all $n \geq m_{0}$. Thus, we conclude that for some $n_{0} \geq m_{0}$, it holds that

$$
\begin{aligned}
\sum_{m=1}^{n} \frac{\log \left(p_{m}\right)}{p_{m}} & \leq \sum_{m=1}^{m_{0}-1} \frac{\log \left(p_{m}\right)}{p_{m}}+\sum_{m=m_{0}}^{n} \frac{\log \left(p_{m}\right)}{p_{m}} \\
& \leq \frac{1}{9} \cdot \log \left(n_{0}\right)+\sum_{m=m_{0}}^{n} \frac{4}{3} \cdot \frac{1}{m} \leq \frac{1}{9} \cdot \log \left(n_{0}\right)+\frac{16}{9} \cdot \log (n) \\
& \leq 2 \cdot \log (n)
\end{aligned}
$$

for all $n \geq n_{0}$, as desired. The final portion of Proposition 1.6 follows formally.

Proposition 1.7. (Weighted Averages) Let $E$ be a nonempty finite set, $n$ a positive integer. For $e \in E$, let $\lambda_{e} \in \mathbb{R}_{>0}, \beta_{e} \in \mathbb{R}$. Then, for any $i=1, \ldots, n$, we have:

$$
\frac{\sum_{\vec{e} \in E^{n}} \beta_{\vec{e}} \cdot \lambda_{\Pi \vec{e}}}{\sum_{\vec{e} \in E^{n}} \lambda_{\Pi \vec{e}}}=\frac{\sum_{\vec{e} \in E^{n}} n \cdot \beta_{e_{i}} \cdot \lambda_{\Pi \vec{e}}}{\sum_{\vec{e} \in E^{n}} \lambda_{\Pi \vec{e}}}=n \cdot \beta_{\mathrm{avg}}
$$

- where we write $\beta_{\mathrm{avg}} \stackrel{\text { def }}{=} \beta_{E} / \lambda_{E}, \quad \beta_{E} \stackrel{\text { def }}{=} \sum_{e \in E} \beta_{e} \cdot \lambda_{e}, \quad \lambda_{E} \stackrel{\text { def }}{=} \sum_{e \in E} \lambda_{e}$,

$$
\beta_{\vec{e}} \stackrel{\text { def }}{=} \sum_{j=1}^{n} \beta_{e_{j}} ; \quad \lambda_{\Pi \vec{e}} \stackrel{\text { def }}{=} \prod_{j=1}^{n} \lambda_{e_{j}}
$$

for any $n$-tuple $\vec{e}=\left(e_{1}, \ldots, e_{n}\right) \in E^{n}$ of elements of $E$.

Proof. We begin by observing that

$$
\lambda_{E}^{n}=\sum_{\vec{e} \in E^{n}} \lambda_{\Pi \vec{e}} ; \quad \beta_{E} \cdot \lambda_{E}^{n-1}=\sum_{\vec{e} \in E^{n}} \beta_{e_{i}} \cdot \lambda_{\Pi \vec{e}}
$$


for any $i=1, \ldots, n$. Thus, summing over $i$, we obtain that

$$
n \cdot \beta_{E} \cdot \lambda_{E}^{n-1}=\sum_{\vec{e} \in E^{n}} \beta_{\vec{e}} \cdot \lambda_{\Pi \vec{e}}=\sum_{\vec{e} \in E^{n}} n \cdot \beta_{e_{i}} \cdot \lambda_{\Pi \vec{e}}
$$

and hence that

$$
\begin{aligned}
n \cdot \beta_{\mathrm{avg}}=n \cdot \beta_{E} \cdot \lambda_{E}^{n-1} / \lambda_{E}^{n} & =\left(\sum_{\vec{e} \in E^{n}} \beta_{\vec{e}} \cdot \lambda_{\Pi \vec{e}}\right) \cdot\left(\sum_{\vec{e} \in E^{n}} \lambda_{\Pi \vec{e}}\right)^{-1} \\
& =\left(\sum_{\vec{e} \in E^{n}} n \cdot \beta_{e_{i}} \cdot \lambda_{\Pi \vec{e}}\right) \cdot\left(\sum_{\vec{e} \in E^{n}} \lambda_{\Pi \vec{e}}\right)^{-1}
\end{aligned}
$$

as desired.

Remark 1.7.1. In Theorem 1.10 below, we shall apply Proposition 1.7 to compute various packet-normalized log-volumes of the sort discussed in [IUTchIII], Proposition 3.9, (i) - i.e., log-volumes normalized by means of the normalized weights discussed in [IUTchIII], Remark 3.1.1, (ii). Here, we recall that the normalized weights discussed in [IUTchIII], Remark 3.1.1, (ii), were computed relative to the non-normalized log-volumes of [AbsTopIII], Proposition 5.8, (iii), (vi) [cf. the discussion of [IUTchIII], Remark 3.1.1, (ii); [IUTchI], Example 3.5, (iii)]. By contrast, in the discussion of the present $\S 1$, our computations are performed relative to normalized log-volumes as discussed in Proposition 1.4, (i). In particular, it follows that the weights $\left[K_{\underline{v}}:\left(F_{\bmod }\right)_{v}\right]^{-1}$, where $\underline{\mathbb{V}} \ni \underline{v} \mid v \in \mathbb{V}_{\text {mod }}$, of the discussion of [IUTchIII], Remark 3.1.1, (ii), must be replaced - i.e., when one works with normalized log-volumes as in Proposition 1.4, (i) — by the weights

$$
\left[K_{\underline{v}}: \mathbb{Q}_{v_{\mathbb{Q}}}\right] \cdot\left[K_{\underline{v}}:\left(F_{\bmod }\right)_{v}\right]^{-1}=\left[\left(F_{\bmod }\right)_{v}: \mathbb{Q}_{v_{\mathbb{Q}}}\right]
$$

- where $\mathbb{V}_{\text {mod }} \ni v \mid v_{\mathbb{Q}} \in \mathbb{V}_{\mathbb{Q}}$. This means that the normalized weights of the final display of [IUTchIII], Remark 3.1.1, (ii), must be replaced, when one works with normalized log-volumes as in Proposition 1.4, (i), by the normalized weights

$$
\frac{\left(\prod_{\alpha \in A}\left[\left(F_{\text {mod }}\right)_{v_{\alpha}}: \mathbb{Q}_{v_{\mathbb{Q}}}\right]\right)}{\sum_{\left\{w_{\alpha}\right\}_{\alpha \in A}}\left(\prod_{\alpha \in A}\left[\left(F_{\bmod }\right)_{w_{\alpha}}: \mathbb{Q}_{v_{\mathbb{Q}}}\right]\right)}
$$

- where the sum is over all collections $\left\{w_{\alpha}\right\}_{\alpha \in A}$ of [not necessarily distinct!] elements $w_{\alpha} \in \mathbb{V}_{\text {mod }}$ lying over $v_{\mathbb{Q}}$ and indexed by $\alpha \in A$. Thus, in summary, when one works with normalized log-volumes as in Proposition 1.4, (i), the appropriate normalized weights are given by the expressions

$$
\frac{\lambda_{\Pi \vec{e}^{\dagger}}}{\sum_{\vec{e} \in E^{n}} \lambda_{\Pi \vec{e}}}
$$

[where $\vec{e}^{\dagger} \in E^{n}$ ] that appear in Proposition 1.7. Here, one takes " $E$ " to be the set of elements of $\underline{\mathbb{V}} \stackrel{\sim}{\rightarrow} \mathbb{V}_{\text {mod }}$ lying over a fixed $v_{\mathbb{Q}}$; one takes " $n$ " to be the cardinality 
of $A$, so that one can write $A=\left\{\alpha_{1}, \ldots, \alpha_{n}\right\}$ [where the $\alpha_{i}$ are distinct]; if $e \in E$ corresponds to $\underline{v} \in \underline{\mathbb{V}}, v \in \mathbb{V}_{\text {mod }}$, then one takes

$$
" \lambda_{e} " \stackrel{\text { def }}{=}\left[\left(F_{\text {mod }}\right)_{v}: \mathbb{Q}_{v_{\mathbb{Q}}}\right] \in \mathbb{R}_{>0}
$$

and " $\beta_{e}$ " to be a normalized log-volume of some compact open subset of $K_{\underline{v}}$.

Before proceeding, we recall some well-known elementary facts concerning elliptic curves. In the following, we shall write $\mathcal{M}_{\text {ell }}$ for the moduli stack of elliptic curves over $\mathbb{Z}$ and

$$
\mathcal{M}_{\text {ell }} \subseteq \overline{\mathcal{M}}_{\text {ell }}
$$

for the natural compactification of $\mathcal{M}_{\text {ell }}$, i.e., the moduli stack of one-dimensional semi-abelian schemes over $\mathbb{Z}$. Also, if $R$ is a $\mathbb{Z}$-algebra, then we shall write $\left(\mathcal{M}_{\text {ell }}\right)_{R} \stackrel{\text { def }}{=}$ $\mathcal{M}_{\text {ell }} \times_{\mathbb{Z}} R,\left(\overline{\mathcal{M}}_{\text {ell }}\right)_{R} \stackrel{\text { def }}{=} \overline{\mathcal{M}}_{\text {ell }} \times_{\mathbb{Z}} R$.

Proposition 1.8. (Torsion Points of Elliptic Curves) Let $k$ be a perfect field $k, \bar{k}$ an algebraic closure of $k$. Write $G_{k} \stackrel{\text { def }}{=} \operatorname{Gal}(\bar{k} / k)$.

(i) Let $l \geq 3$ be a prime number that is invertible in $k$; suppose that $\bar{k}=k$. Let $A$ be an abelian variety over $k$, equipped with a polarization $\lambda$. Write $A[l] \subseteq A(k)$ for the group of l-torsion points of $A(k)$. Then the natural map

$$
\phi: \operatorname{Aut}_{k}(A, \lambda) \rightarrow \operatorname{Aut}(A[l])
$$

from the group of automorphisms of the polarized abelian variety $(A, \lambda)$ over $k$ to the group of automorphisms of the abelian group $A[l]$ is injective.

(ii) Let $E_{\bar{k}}$ be an elliptic curve over $\bar{k}$ with origin $\epsilon_{E} \in E(\bar{k})$. For $n$ a positive integer, write $E_{\bar{k}}[n] \subseteq E_{\bar{k}}(\bar{k})$ for the $G_{k}$-module of $n$-torsion points of $E_{\bar{k}}(\bar{k})$ and

$$
\operatorname{Aut}_{\bar{k}}\left(E_{\bar{k}}\right) \subseteq \operatorname{Aut}_{k}\left(E_{\bar{k}}\right)
$$

for the respective groups of $\epsilon_{E}$-preserving automorphisms of the $\bar{k}$-scheme $E_{\bar{k}}$ and the $k$-scheme $E_{\bar{k}}$. Then we have a natural exact sequence

$$
1 \longrightarrow \operatorname{Aut}_{\bar{k}}\left(E_{\bar{k}}\right) \longrightarrow \operatorname{Aut}_{k}\left(E_{\bar{k}}\right) \longrightarrow G_{k}
$$

- where the image $G_{E} \subseteq G_{k}$ of the homomorphism $\operatorname{Aut}_{k}\left(E_{\bar{k}}\right) \rightarrow G_{k}$ is open and a natural representation

$$
\rho_{n}: \operatorname{Aut}_{k}\left(E_{\bar{k}}\right) \rightarrow \operatorname{Aut}\left(E_{\bar{k}}[n]\right)
$$

on the $n$-torsion points of $E_{\bar{k}}$. The finite extension $k_{E}$ of $k$ determined by $G_{E}$ is the minimal field of definition of $E_{\bar{k}}$, i.e., the field generated over $k$ by the $\boldsymbol{j}$ invariant of $E_{\bar{k}}$. Finally, if $H \subseteq G_{k}$ is any closed subgroup, which corresponds to an extension $k_{H}$ of $k$, then the datum of a model of $E_{\bar{k}}$ over $k_{H}$ [i.e., descent data for $E_{\bar{k}}$ from $\bar{k}$ to $k_{H}$ ] is equivalent to the datum of a section of the homomorphism 
$\operatorname{Aut}_{k}\left(E_{\bar{k}}\right) \rightarrow G_{k}$ over $H$. In particular, the homomorphism $\operatorname{Aut}_{k}\left(E_{\bar{k}}\right) \rightarrow G_{k}$ admits a section over $G_{E}$.

(iii) In the situation of (ii), suppose further that $\operatorname{Aut}_{\bar{k}}\left(E_{\bar{k}}\right)=\{ \pm 1\}$. Then the representation $\rho_{2}$ factors through $G_{E}$ and hence defines a natural representation $G_{E} \rightarrow \operatorname{Aut}\left(E_{\bar{k}}[2]\right)$.

(iv) In the situation of (ii), suppose further that $l \geq 3$ is a prime number that is invertible in $k$, and that $E_{\bar{k}}$ descends to elliptic curves $E_{k}^{\prime}$ and $E_{k}^{\prime \prime}$ over $k$, all of whose l-torsion points are rational over $k$. Then $E_{k}^{\prime}$ is isomorphic to $E_{k}^{\prime \prime}$ over $k$.

(v) In the situation of (ii), suppose further that $k$ is a complete discrete valuation field with ring of integers $\mathcal{O}_{k}$, that $l \geq 3$ is a prime number that is invertible in $\mathcal{O}_{k}$, and that $E_{\bar{k}}$ descends to an elliptic curve $E_{k}$ over $k$, all of whose $l$-torsion points are rational over $k$. Then $E_{k}$ has semi-stable reduction over $\mathcal{O}_{k}$ [i.e., extends to a semi-abelian scheme over $\mathcal{O}_{k}$ ].

(vi) In the situation of (iii), suppose further that 2 is invertible in $k$, that $G_{E}=G_{k}$, and that the representation $G_{E} \rightarrow \operatorname{Aut}\left(E_{\bar{k}}[2]\right)$ is trivial. Then $E_{\bar{k}}$ descends to an elliptic curve $E_{k}$ over $k$ which is defined by means of the Legendre form of the Weierstrass equation [cf., e.g., the statement of Corollary 2.2, below]. If, moreover, $k$ is a complete discrete valuation field with ring of integers $\mathcal{O}_{k}$ such that 2 is invertible in $\mathcal{O}_{k}$, then $E_{k}$ has semi-stable reduction over $\mathcal{O}_{k^{\prime}}$ [i.e., extends to a semi-abelian scheme over $\mathcal{O}_{k^{\prime}}$ ] for some finite extension $k^{\prime} \subseteq \bar{k}$ of $k$ such that $\left[k^{\prime}: k\right] \leq 2$; if $E_{k}$ has good reduction over $\mathcal{O}_{k^{\prime}}$ [i.e., extends to an abelian scheme over $\left.\mathcal{O}_{k^{\prime}}\right]$, then one may in fact take $k^{\prime}$ to be $k$.

(vii) In the situation of (ii), suppose further that $k$ is a complete discrete valuation field with ring of integers $\mathcal{O}_{k}$, that $E_{\bar{k}}$ descends to an elliptic curve $E_{k}$ over $k$, and that $n$ is invertible in $\mathcal{O}_{k}$. If $E_{k}$ has good reduction over $\mathcal{O}_{k}$ [i.e., extends to an abelian scheme over $\left.\mathcal{O}_{k}\right]$, then the action of $G_{k}$ on $E_{\bar{k}}[n]$ is unramified. If $E_{k}$ has bad multiplicative reduction over $\mathcal{O}_{k}$ [i.e., extends to a non-proper semi-abelian scheme over $\left.\mathcal{O}_{k}\right]$, then the action of $G_{k}$ on $E_{\bar{k}}[n]$ is tamely ramified.

Proof. First, we consider assertion (i). Suppose that $\phi$ is not injective. Since $\operatorname{Aut}_{k}(A, \lambda)$ is well-known to be finite [cf., e.g., [Milne], Proposition 17.5, (i)], we thus conclude that there exists an $\alpha \in \operatorname{Ker}(\phi)$ of order $n \neq 1$. We may assume without loss of generality that $n$ is prime. Now we follow the argument of [Milne], Proposition 17.5, (ii). Since $\alpha$ acts trivially on $A[l]$, it follows immediately that the

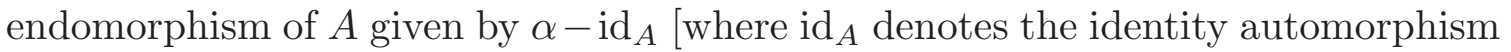
of $A$ ] may be written in the form $l \cdot \beta$, for $\beta$ an endomorphism of $A$ over $k$. Write $T_{l}(A)$ for the l-adic Tate module of $A$. Since $\alpha^{n}=\mathrm{id}_{A}$, it follows that the eigenvalues of the action of $\alpha$ on $T_{l}(A)$ are $n$-th roots of unity. On the other hand, the eigenvalues of the action of $\beta$ on $T_{l}(A)$ are algebraic integers [cf. [Milne], Theorem 12.5]. We thus conclude that each eigenvalue $\zeta$ of the action of $\alpha$ on $T_{l}(A)$ is an $n$-th root of unity which, as an algebraic integer, is $\equiv 1(\bmod l)$ [where $l \geq 3$ ], hence $=1$. Since $\alpha^{n}=\mathrm{id}_{A}$, it follows that $\alpha$ acts on $T_{l}(A)$ as a semi-simple matrix which is also unipotent, hence equal to the identity matrix. But this implies that 
$\alpha=\operatorname{id}_{A}$ [cf. [Milne], Theorem 12.5]. This contradiction completes the proof of assertion (i).

Next, we consider assertion (ii). Since $E_{\bar{k}}$ is proper over $\bar{k}$, it follows [by considering the space of global sections of the structure sheaf of $E_{\bar{k}}$ ] that any automorphism of the scheme $E_{\bar{k}}$ lies over an automorphism of $\bar{k}$. This implies the existence of a natural exact sequence and natural representation as in the statement of assertion (ii). The relationship between $k_{E}$ and the $j$-invariant of $E_{\bar{k}}$ follows immediately from the well-known theory of the $j$-invariant of an elliptic curve [cf., e.g., [Silv], Chapter III, Proposition 1.4, (b), (c)]. The final portion of assertion (ii) concerning models of $E_{\bar{k}}$ follows immediately from the definitions. This completes the proof of assertion (ii). Assertion (iii) follows immediately from the fact that $\{ \pm 1\}$ acts trivially on $E_{\bar{k}}[2]$.

Next, we consider assertion (iv). First, let us observe that it follows immediately from the final portion of assertion (ii) that a model $E_{k}^{*}$ of $E_{\bar{k}}$ over $k$ all of whose $l$-torsion points are rational over $k$ corresponds to a closed subgroup $H^{*} \subseteq \operatorname{Aut}_{k}\left(E_{\bar{k}}\right)$ that lies in the kernel of $\rho_{l}$ and, moreover, maps isomorphically to $G_{k}$. On the other hand, it follows from assertion (i) that the restriction of $\rho_{l}$ to $\operatorname{Aut}_{\bar{k}}\left(E_{\bar{k}}\right) \subseteq \operatorname{Aut}_{k}\left(E_{\bar{k}}\right)$ is trivial. Thus, a closed subgroup $H^{*} \subseteq \operatorname{Aut}_{k}\left(E_{\bar{k}}\right)$ is uniquely determined by the condition that it lie in the kernel of $\rho_{l}$ and, moreover, map isomorphically to $G_{k}$. This completes the proof of assertion (iv).

Next, we consider assertion (v). First, let us observe that, by considering $l$ level structures, we obtain a finite covering of $S \rightarrow\left(\overline{\mathcal{M}}_{\mathrm{ell}}\right)_{\mathbb{Z}\left[\frac{1}{l}\right]}$ which is étale over $\left(\mathcal{M}_{\text {ell }}\right)_{\mathbb{Z}\left[\frac{1}{l}\right]}$ and tamely ramified over the divisor at infinity. Then it follows from assertion (i) that the algebraic stack $S$ is in fact a scheme, which is, moreover, proper over $\mathbb{Z}\left[\frac{1}{l}\right]$. Thus, it follows from the valuative criterion for properness that any $k$-valued point of $S$ determined by $E_{k}$ - where we observe that such a point necessarily exists, in light of our assumption that the $l$-torsion points of $E_{k}$ are rational over $k$ - extends to an $\mathcal{O}_{k}$-valued point of $S$, hence also of $\overline{\mathcal{M}}_{\text {ell }}$, as desired. This completes the proof of assertion (v).

Next, we consider assertion (vi). Since $G_{E}=G_{k}$, it follows from assertion (ii) that $E_{\bar{k}}$ descends to an elliptic curve $E_{k}$ over $k$. Our assumption that the representation $G_{k}=G_{E} \rightarrow \operatorname{Aut}\left(E_{\bar{k}}[2]\right)$ of assertion (iii) is trivial implies that the 2-torsion points of $E_{k}$ are rational over $k$. Thus, by considering suitable global sections of tensor powers of the line bundle on $E_{k}$ determined by the origin on which the automorphism " -1 " of $E_{k}$ acts via multiplication by \pm 1 [cf., e.g., [Harts], Chapter IV, the proof of Proposition 4.6], one concludes immediately that a suitable [possibly trivial] twist $E_{k}^{\prime}$ of $E_{k}$ over $k$ [i.e., such that $E_{k}^{\prime}$ and $E_{k}$ are isomorphic over some quadratic extension $k^{\prime}$ of $k$ ] may be defined by means of the Legendre form of the Weierstrass equation. Now suppose that $k$ is a complete discrete valuation field with ring of integers $\mathcal{O}_{k}$ such that 2 is invertible in $\mathcal{O}_{k}$, and that $E_{k}$ is defined by means of the Legendre form of the Weierstrass equation. Then the fact that $E_{k}$ has semi-stable reduction over $\mathcal{O}_{k^{\prime}}$ for some finite extension $k^{\prime} \subseteq \bar{k}$ of $k$ such that $\left[k^{\prime}: k\right] \leq 2$ follows from the explicit computations of the proof of [Silv], Chapter VII, Proposition 5.4, (c). These explicit computations also imply that if $E_{k}$ has good reduction over $\mathcal{O}_{k^{\prime}}$, then one may in fact take $k^{\prime}$ to be $k$. This completes the proof of assertion (vi). 
Assertion (vii) follows immediately from [NerMod], §7.4, Theorem 5, in the case of good reduction and from [NerMod], $\S 7.4$, Theorem 6, in the case of bad multiplicative reduction.

We are now ready to apply the elementary computations discussed above to give more explicit log-volume estimates for $\Theta$-pilot objects. We begin by recalling some notation and terminology from [GenEll], $§ 1$.

Definition 1.9. Let $F$ be a number field [i.e., a finite extension of the rational number field $\mathbb{Q}]$, whose set of valuations we denote by $\mathbb{V}(F)$. Thus, $\mathbb{V}(F)$ decomposes as a disjoint union $\mathbb{V}(F)=\mathbb{V}(F)^{\text {non }} \cup \mathbb{V}(F)^{\text {arc }}$ of nonarchimedean and archimedean valuations. If $v \in \mathbb{V}(F)$, then we shall write $F_{v}$ for the completion of $F$ at $v$; if $v \in \mathbb{V}(F)^{\text {non }}$, then we shall write $\operatorname{ord}_{v}(-): F_{v}^{\times} \rightarrow \mathbb{Z}$ for the order defined by $v, e_{v}$ for the ramification index of $F_{v}$ over $\mathbb{Q}_{p_{v}}$, and $q_{v}$ for the cardinality of the residue field of $F_{v}$.

(i) $\mathrm{A}(\mathrm{n}) \mathbb{Q}$-Jarithmetic divisor $\mathfrak{a}$ on $F$ is defined to be a finite formal sum

$$
\sum_{v \in \mathbb{V}(F)} c_{v} \cdot v
$$

- where $c_{v} \in \mathbb{Q}$ if $v \in \mathbb{V}(F)^{\text {non }}$ and $c_{v} \in \mathbb{R}$ if $v \in \mathbb{V}(F)^{\text {arc }}$. Here, we shall refer to the set

$$
\operatorname{Supp}(\mathfrak{a})
$$

of $v \in \mathbb{V}(F)$ such that $c_{v} \neq 0$ as the support of $\mathfrak{a}$; if all of the $c_{v}$ are $\geq 0$, then we shall say that the arithmetic divisor is effective. Thus, the $[\mathbb{Q}-]$ arithmetic divisors on $F$ naturally form a group $\operatorname{ADiv}_{\mathbb{Q}}(F)$. The assignment

$$
\mathbb{V}(F)^{\text {non }} \ni v \mapsto \log \left(q_{v}\right) ; \quad \mathbb{V}(F)^{\operatorname{arc}} \ni v \mapsto 1
$$

determines a homomorphism

$$
\operatorname{deg}_{F}: \operatorname{ADiv}_{\mathbb{Q}}(F) \rightarrow \mathbb{R}
$$

which we shall refer to as the degree map. If $\mathfrak{a} \in \operatorname{ADiv}_{\mathbb{Q}}(F)$, then we shall refer to

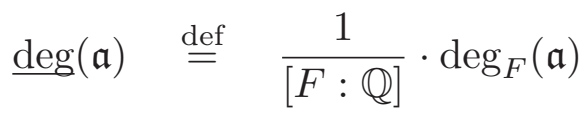

as the normalized degree of $\mathfrak{a}$. Thus, for any finite extension $K$ of $F$, we have

$$
\underline{\operatorname{deg}}\left(\left.\mathfrak{a}\right|_{K}\right)=\underline{\operatorname{deg}}(\mathfrak{a})
$$

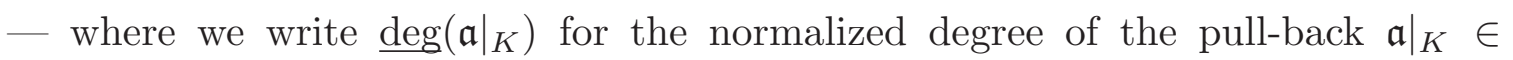
$\operatorname{ADiv}_{\mathbb{Q}}(K)$ [defined in the evident fashion] of $\mathfrak{a}$ to $K$.

(ii) Let $v_{\mathbb{Q}} \in \mathbb{V}_{\mathbb{Q}} \stackrel{\text { def }}{=} \mathbb{V}(\mathbb{Q}), E \subseteq \mathbb{V}(F)$ a nonempty set of elements lying over $v_{\mathbb{Q}}$. If $\mathfrak{a}=\sum_{v \in \mathbb{V}(F)} c_{v} \cdot v \in \operatorname{ADiv}_{\mathbb{Q}}(F)$, then we shall write

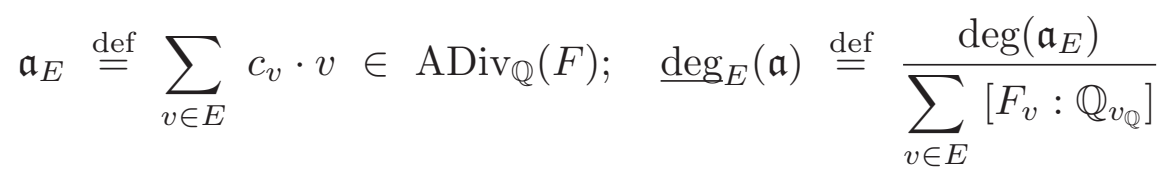


for the portion of $\mathfrak{a}$ supported in $E$ and the "normalized $E$-degree" of $\mathfrak{a}$, respectively. Thus, for any finite extension $K$ of $F$, we have

$$
\underline{\operatorname{deg}}_{\left.E\right|_{K}}\left(\left.\mathfrak{a}\right|_{K}\right)=\underline{\operatorname{deg}}_{E}(\mathfrak{a})
$$

- where we write $\left.E\right|_{K} \subseteq \mathbb{V}(K)$ for the set of valuations lying over valuations $\in E$.

\section{Theorem 1.10. (Log-volume Estimates for $\Theta$-Pilot Objects) Set}

$$
\epsilon_{\Theta} \stackrel{\text { def }}{=} \min \left(\frac{40 \eta_{\text {prm }}}{3}, 3^{-210}\right) \in \mathbb{R}_{>0}
$$

- where the constant $\eta_{\text {prm }} \in \mathbb{R}_{>0}$ is as in Proposition 1.6. Then the constant $\epsilon_{\Theta} \in \mathbb{R}_{>0}$ satisfies the following property:

Fix a collection of initial $\Theta$-data as in [IUTchI], Definition 3.1. Suppose that we are in the situation of [IUTchIII], Corollary 3.12. Also, in the notation of [IUTchI], Definition 3.1, let us write $d_{\mathrm{mod}} \stackrel{\text { def }}{=}\left[F_{\mathrm{mod}}: \mathbb{Q}\right]$ and

$$
F_{\text {mod }} \subseteq F_{\text {tpd }} \stackrel{\text { def }}{=} F_{\text {mod }}\left(E_{F_{\text {mod }}}[2]\right) \subseteq F
$$

for the "tripodal" intermediate field obtained from $F_{\bmod }$ by adjoining the fields of definition of the 2-torsion points of any model of $E_{F}$ over $F_{\bmod }$ [cf. Proposition 1.8, (ii), (iii)]. Moreover, we assume that the (3.5)-torsion points of $E_{F}$ are defined over $F$, and that

$$
F=F_{\text {mod }}\left(\sqrt{-1}, E_{F_{\text {mod }}}[2 \cdot 3 \cdot 5]\right) \stackrel{\text { def }}{=} F_{\text {tpd }}\left(\sqrt{-1}, E_{F_{\text {tpd }}}[3 \cdot 5]\right)
$$

- i.e., that $F$ is obtained from $F_{\mathrm{tpd}}$ by adjoining $\sqrt{-1}$, together with the fields of definition of the $(3 \cdot 5)$-torsion points of a model $E_{F_{\mathrm{tpd}}}$ of the elliptic curve $E_{F}$ over $F_{\text {tpd }}$ determined by the Legendre form of the Weierstrass equation [cf., e.g., the statement of Corollary 2.2, below; Proposition 1.8, (vi)]. [Thus, it follows from Proposition 1.8, (iv), that $E_{F} \cong E_{F_{\mathrm{tpd}}} \times_{F_{\mathrm{tpd}}} F$ over $F$.] If $F_{\mathrm{mod}} \subseteq F_{\square} \subseteq K$ is any intermediate extension which is Galois over $F_{\mathrm{mod}}$, then we shall write

$$
\mathfrak{d}_{\mathrm{ADiv}}^{F} \in \operatorname{ADiv}_{\mathbb{Q}}\left(F_{\square}\right)
$$

for the effective divisor determined by the different ideal of $F_{\square}$ over $\mathbb{Q}$,

$$
\mathfrak{q}_{\mathrm{ADiv}}^{F_{\square}} \in \operatorname{ADiv}_{\mathbb{Q}}\left(F_{\square}\right)
$$

for the effective arithmetic divisor determined by the $\boldsymbol{q}$-parameters of the elliptic curve $E_{F}$ at the elements of $\mathbb{V}\left(F_{\square}\right)^{\text {bad }} \stackrel{\text { def }}{=} \mathbb{V}_{\bmod }^{\text {bad }} \times_{\mathbb{V}_{\bmod }} \mathbb{V}\left(F_{\square}\right)(\neq \emptyset)$,

$$
\mathfrak{f}_{\mathrm{ADiv}}^{F_{\square}} \in \operatorname{ADiv}_{\mathbb{Q}}\left(F_{\square}\right)
$$

for the effective arithmetic divisor whose support coincides with $\operatorname{Supp}\left(\mathfrak{q}_{\mathrm{ADiv}}^{F_{\square}}\right)$, but all of whose coefficients are equal to 1 - i.e., the conductor - and

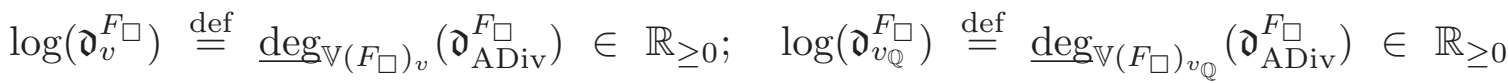

$$
\begin{aligned}
& \log \left(\mathfrak{d}^{F} \square\right) \stackrel{\text { def }}{=} \underline{\operatorname{deg}}\left(\mathfrak{d}_{\text {ADiv }}^{F_{\square}}\right) \in \mathbb{R}_{\geq 0}
\end{aligned}
$$




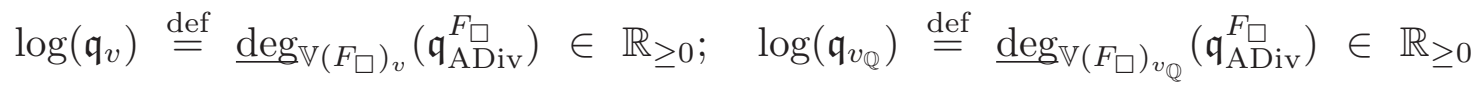

$$
\begin{aligned}
& \log (\mathfrak{q}) \stackrel{\text { def }}{=} \underline{\operatorname{deg}}\left(\mathfrak{q}_{\text {ADiv }}^{F_{\square}}\right) \in \mathbb{R}_{\geq 0}
\end{aligned}
$$

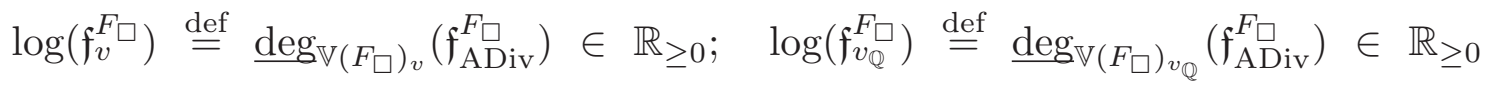

$$
\begin{aligned}
& \log \left(\mathfrak{f}^{F \square}\right) \stackrel{\text { def }}{=} \underline{\operatorname{deg}}\left(\mathfrak{f}_{\mathrm{AD} \text { iv }}^{F_{\square}}\right) \in \mathbb{R}_{\geq 0}
\end{aligned}
$$

- where $v \in \mathbb{V}_{\text {mod }}=\mathbb{V}\left(F_{\text {mod }}\right), v_{\mathbb{Q}} \in \mathbb{V}_{\mathbb{Q}}=\mathbb{V}(\mathbb{Q}), \mathbb{V}\left(F_{\square}\right)_{v} \stackrel{\text { def }}{=} \mathbb{V}\left(F_{\square}\right) \times_{\mathbb{V}_{\text {mod }}}\{v\}$, $\mathbb{V}\left(F_{\square}\right)_{v_{\mathbb{Q}}} \stackrel{\text { def }}{=} \mathbb{V}\left(F_{\square}\right) \times_{\mathbb{V}_{\mathbb{Q}}}\left\{v_{\mathbb{Q}}\right\}$. Here, we observe that the various $\log \left(\mathfrak{q}_{(-)}\right)$'s" are independent of the choice of $F_{\square}$, and that the quantity $|\log (\underline{q})| \in \mathbb{R}_{>0}$ " defined in [IUTchIII], Corollary 3.12, is equal to $\frac{1}{2 l} \cdot \log (\mathfrak{q}) \in \mathbb{R}[$ cf. the definition of " $\underline{\underline{q}}$ " in [IUTchI], Example 3.2, (iv)]. Then one may take the constant " $C_{\Theta} \in \mathbb{R}$ " [IUTchIII], Corollary 3.12, to be

$$
\frac{l+1}{4} \cdot\left\{\left(1+\epsilon+\frac{28 \cdot d_{\mathrm{mod}}}{l}\right) \cdot\left(\log \left(\mathfrak{d}^{F_{\mathrm{tpd}}}\right)+\log \left(\mathfrak{f}^{F_{\mathrm{tpd}}}\right)\right)+2 \cdot \log \left(l \cdot \epsilon^{-7}\right)-\frac{1}{6} \cdot \log (\mathfrak{q})\right\}-1
$$

and hence, by applying [IUTchIII], Corollary 3.12, conclude that

$$
\begin{aligned}
\frac{1}{6} \cdot \log (\mathfrak{q}) & \leq\left(1+\epsilon+\frac{28 \cdot d_{\text {mod }}}{l}\right) \cdot\left(\log \left(\mathfrak{d}^{F_{\mathrm{tpd}}}\right)+\log \left(\mathfrak{f}^{F_{\mathrm{tpd}}}\right)\right)+2 \cdot \log \left(l \cdot \epsilon^{-7}\right) \\
& \leq\left(1+\epsilon+\frac{28 \cdot d_{\bmod }}{l}\right) \cdot\left(\log \left(\mathfrak{d}^{F}\right)+\log \left(\mathfrak{f}^{F}\right)\right)+2 \cdot \log \left(l \cdot \epsilon^{-7}\right)
\end{aligned}
$$

for any $\epsilon \in \mathbb{R}_{>0}$ satisfying $\epsilon<\epsilon_{\Theta}$.

Proof. For ease of reference, we divide our discussion into steps, as follows.

(i) We begin by recalling the following elementary identities for $n \in \mathbb{N}_{\geq 1}$ :

(E1) $\frac{1}{n} \sum_{m=1}^{n} m=\frac{1}{2}(n+1)$;

(E2) $\frac{1}{n} \sum_{m=1}^{n} m^{2}=\frac{1}{6}(2 n+1)(n+1)$.

Also, we recall the following elementary facts:

(E3) For $p$ a prime number, the cardinality $\left|G L_{2}\left(\mathbb{F}_{p}\right)\right|$ of $G L_{2}\left(\mathbb{F}_{p}\right)$ is given by $\left|G L_{2}\left(\mathbb{F}_{p}\right)\right|=p(p+1)(p-1)^{2}$.

(E4) For $p=2,3,5$, the expression of (E3) may be computed as follows: $2(2+1)(2-1)^{2}=2 \cdot 3 ; \quad 3(3+1)(3-1)^{2}=3 \cdot 2^{4} ; \quad 5(5+1)(5-1)^{2}=5 \cdot 2^{5} \cdot 3$.

(E5) The degree of the extension $F_{\bmod }(\sqrt{-1}) / F_{\text {mod }}$ is $\leq 2$.

(E6) We have: $0 \leq \log (2) \leq 1, \quad 1 \leq \log (3) \leq \log (\pi) \leq \log (5) \leq 2$.

(ii) Next, let us observe that the inequality

$$
\log \left(\mathfrak{d}^{F_{\mathrm{tpd}}}\right)+\log \left(\mathfrak{f}^{F_{\mathrm{tpd}}}\right) \leq \log \left(\mathfrak{d}^{F}\right)+\log \left(\mathfrak{f}^{F}\right)
$$


follows immediately from Proposition 1.3, (i), and the various definitions involved. On the other hand, the inequality

$$
\begin{aligned}
\log \left(\mathfrak{d}^{F}\right)+\log \left(\mathfrak{f}^{F}\right) & \leq \log \left(\mathfrak{d}^{F_{\mathrm{tpd}}}\right)+\log \left(\mathfrak{f}^{F_{\mathrm{tpd}}}\right)+\log \left(2^{11} \cdot 3^{3} \cdot 5^{2}\right) \\
& \leq \log \left(\mathfrak{d}^{F_{\mathrm{tpd}}}\right)+\log \left(\mathfrak{f}^{F_{\mathrm{tpd}}}\right)+21
\end{aligned}
$$

follows by applying Proposition 1.3, (i), at the primes that do not divide $2 \cdot 3 \cdot 5$ [over which the extension $F / F_{\text {tpd }}$ is tamely ramified - cf. Proposition 1.8, (vi), (vii)] and applying Proposition 1.3, (ii), together with (E3), (E4), (E5), (E6), and the fact that we have a natural inclusion $\operatorname{Gal}\left(F / F_{\text {tpd }}\right) \hookrightarrow G L_{2}\left(\mathbb{F}_{3}\right) \times G L_{2}\left(\mathbb{F}_{5}\right) \times \mathbb{Z} / 2 \mathbb{Z}$, at the primes that divide $2 \cdot 3 \cdot 5$. In a similar vein, since the extension $K / F$ is tamely ramified at the primes that do not divide $l$, and we have a natural isomorphism $\operatorname{Gal}(K / F) \stackrel{\sim}{\rightarrow} G L_{2}\left(\mathbb{F}_{l}\right)$, the inequality

$$
\begin{aligned}
\log \left(\mathfrak{d}^{K}\right) \leq \log \left(\mathfrak{d}^{K}\right)+\log \left(\mathfrak{f}^{K}\right) & \leq \log \left(\mathfrak{d}^{F}\right)+\log \left(\mathfrak{f}^{F}\right)+2 \cdot \log (l) \\
& \leq \log \left(\mathfrak{d}^{F_{\mathrm{tpd}}}\right)+\log \left(\mathfrak{f}^{F_{\mathrm{tpd}}}\right)+2 \cdot \log (l)+21
\end{aligned}
$$

follows immediately from Proposition 1.3, (i), (ii). Finally, for later reference, we observe that

$$
\left(1+\frac{4}{l}\right) \cdot \log \left(\mathfrak{d}^{K}\right) \leq\left(1+\frac{4}{l}\right) \cdot\left(\log \left(\mathfrak{d}^{F_{\mathrm{tpd}}}\right)+\log \left(\mathfrak{f}^{F_{\mathrm{tpd}}}\right)\right)+2 \cdot \log (l)+46
$$

- where we apply the estimates $\frac{\log (l)}{l} \leq \frac{1}{2}$ and $1+\frac{4}{l} \leq 2$, both of which are consequences of the fact that $l \geq 5$ [cf. also (E6)].

(iii) If $F_{\text {tpd }} \subseteq F_{\square} \subseteq K$ is any intermediate extension which is Galois over $F_{\text {mod }}$, then we shall write

$$
\mathbb{V}\left(F_{\square}\right)^{\mathrm{dst}} \subseteq \mathbb{V}\left(F_{\square}\right)^{\text {non }}
$$

for the set of "distinguished" nonarchimedean valuations $v \in \mathbb{V}\left(F_{\square}\right)^{\text {non }}$, i.e., $v$ that extend to a valuation $\in \mathbb{V}(K)^{\text {non }}$ that ramifies over $\mathbb{Q}$. Now observe that it follows immediately from Proposition 1.8, (vi), (vii), that

(D0) if $v \in \mathbb{V}\left(F_{\mathrm{tpd}}\right)^{\text {non }}$ does not divide $2 \cdot 3 \cdot 5 \cdot l$ and, moreover, is not contained in $\operatorname{Supp}\left(\mathfrak{q}_{\mathrm{ADiv}}^{F_{\mathrm{tpd}}}\right)$, then the extension $K / F_{\text {tpd }}$ is unramified over $v$.

Also, let us recall [cf. the various definitions involved] that $K$ contains a primitive $4 \cdot 3 \cdot 5 \cdot$ l-th root of unity, hence is ramified over $\mathbb{Q}$ at any valuation $\in \mathbb{V}(K)^{\text {non }}$ that divides $2 \cdot 3 \cdot 5 \cdot l$. Thus, one verifies immediately [i.e., by applying (D0); cf. also [IUTchI], Definition 3.1, (c)] that the following conditions on a valuation $v \in \mathbb{V}\left(F_{\square}\right)^{\text {non }}$ are equivalent:

(D1) $v \in \mathbb{V}\left(F_{\square}\right)^{\mathrm{dst}}$.

(D2) The valuation $v$ either divides $2 \cdot 3 \cdot 5 \cdot l$ or lies in $\operatorname{Supp}\left(\mathfrak{q}_{\mathrm{ADiv}}^{F_{\square}}+\mathfrak{d}_{\mathrm{ADiv}}^{F_{\square}}\right)$.

(D3) The image of $v$ in $\mathbb{V}\left(F_{\mathrm{tpd}}\right)$ lies in $\mathbb{V}\left(F_{\mathrm{tpd}}\right)^{\mathrm{dst}}$.

Let us write

$$
\mathbb{V}_{\text {mod }}^{\text {dst }} \subseteq \mathbb{V}_{\text {mod }}^{\text {non }} ; \quad \mathbb{V}_{\mathbb{Q}}^{\text {dst }} \subseteq \mathbb{V}_{\mathbb{Q}}^{\text {non }}
$$


for the respective images of $\mathbb{V}\left(F_{\mathrm{tpd}}\right)^{\mathrm{dst}}$ in $\mathbb{V}_{\text {mod }}, \mathbb{V}_{\mathbb{Q}}$ and, for $F_{*} \in\left\{F_{\square}, F_{\text {mod }}, \mathbb{Q}\right\}$ and $v_{\mathbb{Q}} \in \mathbb{V}_{\mathbb{Q}}$,

$$
\begin{aligned}
& \mathfrak{s}_{\mathrm{ADiv}}^{F_{*}} \stackrel{\text { def }}{=} \sum_{v \in \mathbb{V}\left(F_{*}\right) \mathrm{dst}} e_{v} \cdot v \in \operatorname{ADiv}_{\mathbb{Q}}\left(F_{*}\right)
\end{aligned}
$$

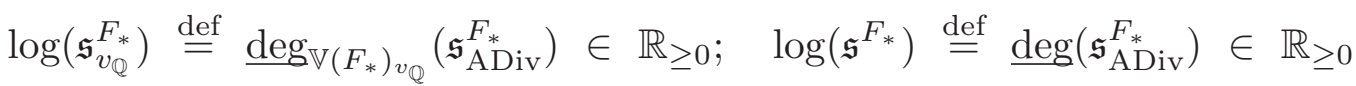

— where we write $\mathbb{V}\left(F_{*}\right)_{v_{\mathbb{Q}}} \stackrel{\text { def }}{=} \mathbb{V}\left(F_{*}\right) \times_{\mathbb{V}_{\mathbb{Q}}}\left\{v_{\mathbb{Q}}\right\}$. One verifies immediately [again, by applying (D0); cf. also [IUTchI], Definition 3.1, (c)] that the following conditions on a valuation $v_{\mathbb{Q}} \in \mathbb{V}_{\mathbb{Q}}^{\text {non }}$ are equivalent:

(D4) $v_{\mathbb{Q}} \in \mathbb{V}_{\mathbb{Q}}^{\text {dst }}$

(D5) The valuation $v_{\mathbb{Q}}$ ramifies in $K$.

(D6) Either $p_{v_{\mathbb{Q}}} \mid 2 \cdot 3 \cdot 5 \cdot l$ or $v_{\mathbb{Q}}$ lies in the image of $\operatorname{Supp}\left(\mathfrak{q}_{\mathrm{ADiv}}^{F_{\mathrm{tpd}}}+\mathfrak{d}_{\mathrm{ADiv}}^{F_{\mathrm{tpd}}}\right)$.

(D7) Either $p_{v_{\mathbb{Q}}} \mid 2 \cdot 3 \cdot 5 \cdot l$ or $v_{\mathbb{Q}}$ lies in the image of $\operatorname{Supp}\left(\mathfrak{q}_{\text {ADiv }}^{F}+\mathfrak{d}_{\text {ADiv }}^{F}\right)$.

Next, for later reference, we observe that the inequality

$$
\frac{1}{p_{v_{\mathbb{Q}}}} \cdot \log \left(\mathfrak{s}_{v_{\mathbb{Q}}}^{F_{\text {mod }}}\right) \leq \frac{1}{p_{v_{\mathbb{Q}}}} \cdot \log \left(p_{v_{\mathbb{Q}}}\right)
$$

holds for any $v_{\mathbb{Q}} \in \mathbb{V}_{\mathbb{Q}}$; in particular, when $p_{v_{\mathbb{Q}}}=l(\geq 5)$, it holds that

$$
\frac{1}{p_{v_{\mathbb{Q}}}} \cdot \log \left(\mathfrak{s}_{v_{\mathbb{Q}}}^{F_{\text {mod }}}\right) \leq \frac{1}{p_{v_{\mathbb{Q}}}} \cdot \log \left(p_{v_{\mathbb{Q}}}\right) \leq \frac{1}{2}
$$

- cf. (E6). On the other hand, it follows immediately from Proposition 1.3, (i), by considering the various possibilities for elements $\in \operatorname{Supp}\left(\mathfrak{s}_{\text {ADiv }}^{F_{\text {mod }}}\right)$, that

$$
\log \left(\mathfrak{s}_{v_{\mathbb{Q}}}^{F_{\text {mod }}}\right) \leq 2 \cdot\left(\log \left(\mathfrak{d}_{v_{\mathbb{Q}}}^{F_{\text {tpd }}}\right)+\log \left(\mathfrak{f}_{v_{\mathbb{Q}}}^{F_{\text {tpd }}}\right)\right)
$$

— and hence that

$$
\frac{1}{p_{v_{\mathbb{Q}}}} \cdot \log \left(\mathfrak{s}_{v_{\mathbb{Q}}}^{F_{\text {mod }}}\right) \leq \frac{2}{p_{v_{\mathbb{Q}}}} \cdot\left(\log \left(\mathfrak{d}_{v_{\mathbb{Q}}}^{F_{\text {tpd }}}\right)+\log \left(\mathfrak{f}_{v_{\mathbb{Q}}}^{F_{\text {tpd }}}\right)\right)
$$

— for any $v_{\mathbb{Q}} \in \mathbb{V}_{\mathbb{Q}}$ such that $p_{v_{\mathbb{Q}}} \notin\{2,3,5, l\}$. In a similar vein, we conclude that

$$
\begin{aligned}
\log \left(\mathfrak{s}^{\mathbb{Q}}\right) & \leq 2 \cdot d_{\mathrm{mod}} \cdot\left(\log \left(\mathfrak{d}^{F_{\mathrm{tpd}}}\right)+\log \left(\mathfrak{f}^{F_{\mathrm{tpd}}}\right)\right)+\log (2 \cdot 3 \cdot 5 \cdot l) \\
& \leq 2 \cdot d_{\mathrm{mod}} \cdot\left(\log \left(\mathfrak{d}^{F_{\mathrm{tpd}}}\right)+\log \left(\mathfrak{f}^{F_{\mathrm{tpd}}}\right)\right)+5+\log (l)
\end{aligned}
$$

and hence that

$$
\frac{12}{l} \cdot \log \left(\mathfrak{s}^{\mathbb{Q}}\right) \leq \frac{24 \cdot d_{\mathrm{mod}}}{l} \cdot\left(\log \left(\mathfrak{d}^{F_{\mathrm{tpd}}}\right)+\log \left(\mathfrak{f}^{F_{\mathrm{tpd}}}\right)\right)+18
$$

- cf. (E6); the fact that $l \geq 5$. Combining this last inequality with the inequality of the final display of Step (ii) yields the inequality

$\left(1+\frac{4}{l}\right) \cdot \log \left(\mathfrak{d}^{K}\right)+\frac{12}{l} \cdot \log \left(\mathfrak{s}^{\mathbb{Q}}\right) \leq\left(1+\frac{28 \cdot d_{\text {mod }}}{l}\right) \cdot\left(\log \left(\mathfrak{d}^{F_{\mathrm{tpd}}}\right)+\log \left(\mathfrak{f}^{F_{\mathrm{tpd}}}\right)\right)+2 \cdot \log (l)+64$

— where we apply the estimate $d_{\bmod } \geq 1$. 
(iv) In order to estimate the constant " $C_{\Theta}$ " of [IUTchIII], Corollary 3.12, we must, according to the various definitions given in the statement of [IUTchIII], Corollary 3.12, compute an upper bound for the

procession-normalized mono-analytic log-volume of the union of the possible images of a $\Theta$-pilot object, relative to the relevant Kummer isomorphisms [cf. [IUTchIII], Theorem 3.11, (ii)], in the multiradial representation of [IUTchIII], Theorem 3.11, (i), which we regard as subject to the indeterminacies (Ind1), (Ind2), (Ind3) described in [IUTchIII], Theorem 3.11, (i), (ii).

Thus, we proceed to estimate this log-volume at each $v_{\mathbb{Q}} \in \mathbb{V}_{\mathbb{Q}}$. Once one fixes $v_{\mathbb{Q}}$, this amounts to estimating the component of this log-volume in

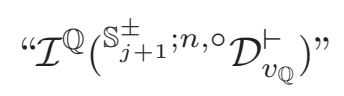

[cf. the notation of [IUTchIII], Theorem 3.11, (i), (a)], for each $j \in\left\{1, \ldots, l^{*}\right\}$, which we shall also regard as an element of $\mathbb{F}_{l}^{*}$, and then computing the average, over $j \in\left\{1, \ldots, l^{*}\right\}$, of these estimates. Here, we recall [cf. [IUTchI], Proposition 6.9, (i); [IUTchIII], Proposition 3.4, (ii)] that $\mathbb{S}_{j+1}^{ \pm}=\{0,1, \ldots, j\}$. Also, we recall

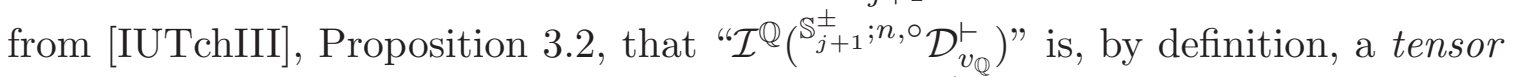
product of $j+1$ copies, indexed by the elements of $\mathbb{S}_{j+1}^{ \pm}$, of the direct sum of the $\mathbb{Q}$ spans of the log-shells associated to each of the elements of $\mathbb{V}\left(F_{\text {mod }}\right)_{v_{\mathbb{Q}}}$ [cf., especially, the second and third displays of [IUTchIII], Proposition 3.2]. In particular, for each collection

$$
\left\{v_{i}\right\}_{i \in \mathbb{S}_{j+1}^{ \pm}}
$$

of [not necessarily distinct!] elements of $\mathbb{V}\left(F_{\bmod }\right)_{v_{\mathbb{Q}}}$, we must estimate the component of the log-volume in question corresponding to the tensor product of the $\mathbb{Q}$-spans of the log-shells associated to this collection $\left\{v_{i}\right\}_{i \in \mathbb{S}_{j+1}^{ \pm}}$and then compute the weighted average [cf. the discussion of Remark 1.7.1], over possible collections $\left\{v_{i}\right\}_{i \in \mathbb{S}_{j+1}^{ \pm}}$, of these estimates.

(v) Let $v_{\mathbb{Q}} \in \mathbb{V}_{\mathbb{Q}}^{\text {dst }}$. Fix $j,\left\{v_{i}\right\}_{i \in \mathbb{S}_{j+1}^{ \pm}}$as in Step (iv). Write $\underline{v}_{i} \in \underline{\mathbb{V}} \stackrel{\sim}{\rightarrow} \mathbb{V}_{\bmod }=$ $\mathbb{V}\left(F_{\text {mod }}\right)$ for the element corresponding to $v_{i}$. We would like to apply Proposition 1.4 , (iii), to the present situation, by taking

- "I" to be $\mathbb{S}_{j+1}^{ \pm}$;

- " $I^{*} \subseteq I$ " to be the set of $i \in I$ such that $\underline{v}_{i} \in \mathbb{V}(K)^{\text {dst }}\left[\right.$ i.e., such that $\underline{v}_{i}$ is ramified over $\mathbb{Q}]$;

- " $k_{i}$ " to be $K_{\underline{v}_{i}}$ [so " $R_{i}$ " will be the ring of integers $\mathcal{O}_{K_{\underline{v}_{i}}}$ of $K_{\underline{v}_{i}}$;

. " $i \dagger$ " to be $j \in \mathbb{S}_{j+1}^{ \pm}$;

. " $\lambda$ " to be 0 if $\underline{v}_{j} \in \underline{\mathbb{V}}^{\text {good }}$

. " $\lambda$ " to be "ord (-)" of the element $\underline{\underline{q}}_{\underline{v}_{j}}^{j^{2}}[\mathrm{cf}$. the definition of " $\underline{\underline{q}} \underline{\underline{v}}$ " in [IUTchI], Example 3.2, (iv)] if $\underline{v}_{j} \in \underline{\mathbb{V}}^{\text {bad }}$. 
Thus, the inclusion " $\phi\left(p^{\lambda} \cdot R_{i^{\dagger}} \otimes_{R_{i \dagger}}\left(R_{I}\right)^{\sim}\right) \subseteq p^{\lfloor\lambda\rfloor-\left\lceil\mathfrak{d}_{I}\right\rceil-\left\lceil a_{I}\right\rceil} \cdot \log _{p}\left(R_{I}^{\times}\right)$" of Proposition 1.4, (iii), implies that the result of multiplying the tensor product of log-shells under consideration by a suitable nonpositive [cf. the final portion of Proposition 1.4, (iii)] integer power of $p_{v_{\mathbb{Q}}}$ contains the "union of possible images of a $\Theta$-pilot object" discussed in Step (iv). That is to say, the indeterminacies (Ind1) and (Ind2) are taken into account by the arbitrary nature of the automorphism " $\phi$ " [cf. Proposition 1.2], while the indeterminacy (Ind3) is taken into account by the fact that we are considering upper bounds [cf. the discussion of Step (x) of the proof of [IUTchIII], Corollary 3.12], together with the fact that the above-mentioned integer power of $p_{v_{\mathbb{Q}}}$ is nonpositive, which implies that the module obtained by multiplying by this power of $p_{v_{\mathbb{Q}}}$ contains the tensor product of log-shells under consideration. Thus, an upper bound on the component of the log-volume under consideration may be obtained by computing an upper bound for the log-volume of the right-hand side " $p^{\lfloor\lambda\rfloor-\left\lceil\mathfrak{o}_{I}\right\rceil-\left\lceil a_{I}\right\rceil} \cdot \log _{p}\left(R_{I}^{\times}\right)$" of the above inclusion. Such an upper bound

$$
\text { "( }\left(-\lambda+\mathfrak{d}_{I}+3+4 \cdot\left|I^{*}\right| / p\right) \cdot \log (p) "
$$

is given in the displayed inequality of Proposition 1.4, (iii). Here, we note that, unlike the other terms that appear in this upper bound, " $\lambda$ " is asymmetric with respect to the choice of " $i^{\dagger} \in I^{\prime}$ " in $\mathbb{S}_{j+1}^{ \pm}$. Since we would like to compute weighted averages [cf. the discussion of Remark 1.7.1], we thus observe that, after symmetrizing with respect to the choice of " $i^{\dagger} \in I$ " in $\mathbb{S}_{j+1}^{ \pm}$, this upper bound may be written in the form

$$
\text { " } \beta_{\vec{e}} "
$$

[cf. the notation of Proposition 1.7] if, in the situation of Proposition 1.7, one takes

- "E" to be $\mathbb{V}\left(F_{\text {mod }}\right)_{v_{\mathbb{Q}}}$;

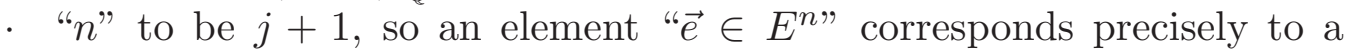
collection $\left\{v_{i}\right\}_{i \in \mathbb{S}_{j+1}^{ \pm}}$;

- " $\lambda_{e}$ ", for an element $e \in E$ corresponding to $v \in \mathbb{V}\left(F_{\text {mod }}\right)=\mathbb{V}_{\text {mod }}$, to be $\left[\left(F_{\text {mod }}\right)_{v}: \mathbb{Q}_{v_{\mathbb{Q}}}\right] \in \mathbb{R}_{>0}$

. " $\beta_{e}$ ", for an element $e \in E$ corresponding to $\underline{v} \in \underline{\mathbb{V}}, v \in \mathbb{V}\left(F_{\text {mod }}\right)=\mathbb{V}$ mod, to be

$$
\log \left(\mathfrak{d}_{v}^{K}\right)-\frac{j^{2}}{2 l(j+1)} \cdot \log \left(\mathfrak{q}_{v}\right)+\frac{3}{j+1} \cdot \log \left(p_{v_{\mathbb{Q}}}\right)+\frac{4 \cdot \iota_{v}}{p_{v_{\mathbb{Q}}}} \cdot \log \left(p_{v_{\mathbb{Q}}}\right)
$$

— where we set $\iota_{v} \stackrel{\text { def }}{=} 1$ if $\underline{v} \in \mathbb{V}(K)^{\text {dst }}, \iota_{v} \stackrel{\text { def }}{=} 0$ if $\underline{v} \in \mathbb{V}(K)^{\mathrm{dst}}$.

Here, we note that it follows immediately from the first equality of the first display of Proposition 1.7 that, after passing to weighted averages, the operation of symmetrizing with respect to the choice of " $i \dagger \in I$ " in $\mathbb{S}_{j+1}^{ \pm}$does not affect the computation of the upper bound under consideration. Thus, by applying Proposition 1.7, we obtain that the resulting "weighted average upper bound" is given by

$$
(j+1) \cdot \log \left(\mathfrak{d}_{v_{\mathbb{Q}}}^{K}\right)-\frac{j^{2}}{2 l} \cdot \log \left(\mathfrak{q}_{v_{\mathbb{Q}}}\right)+3 \cdot \log \left(\mathfrak{s}_{v_{\mathbb{Q}}}^{\mathbb{Q}}\right)+\frac{4(j+1)}{p_{v_{\mathbb{Q}}}} \cdot \log \left(\mathfrak{s}_{v_{\mathbb{Q}}}^{F_{\text {mod }}}\right)
$$

- where we recall the notational conventions introduced in Step (iii). Thus, it remains to compute the average over $j \in \mathbb{F}_{l}^{*}$. By averaging over $j \in\left\{1, \ldots, l^{*}=\right.$ 
$\left.\frac{l-1}{2}\right\}$ and applying (E1), (E2), we obtain the "procession-normalized upper bound"

$$
\begin{aligned}
\frac{\left(l^{*}+3\right)}{2} \cdot & \log \left(\mathfrak{d}_{v_{\mathbb{Q}}}^{K}\right)-\frac{\left(2 l^{*}+1\right)\left(l^{*}+1\right)}{12 l} \cdot \log \left(\mathfrak{q}_{v_{\mathbb{Q}}}\right)+3 \cdot \log \left(\mathfrak{s}_{v_{\mathbb{Q}}}^{\mathbb{Q}}\right)+\frac{2\left(l^{*}+3\right)}{p_{v_{\mathbb{Q}}}} \cdot \log \left(\mathfrak{s}_{v_{\mathbb{Q}}}^{F_{\text {mod }}}\right) \\
& =\frac{l+5}{4} \cdot \log \left(\mathfrak{d}_{v_{\mathbb{Q}}}^{K}\right)-\frac{l+1}{24} \cdot \log \left(\mathfrak{q}_{v_{\mathbb{Q}}}\right)+3 \cdot \log \left(\mathfrak{s}_{v_{\mathbb{Q}}}^{\mathbb{Q}}\right)+\frac{(l+5)}{p_{v_{\mathbb{Q}}}} \cdot \log \left(\mathfrak{s}_{v_{\mathbb{Q}}}^{F_{\text {mod }}}\right) \\
& \leq \frac{l+1}{4} \cdot\left\{\left(1+\frac{4}{l}\right) \cdot \log \left(\mathfrak{d}_{v_{\mathbb{Q}}}^{K}\right)-\frac{1}{6} \cdot \log \left(\mathfrak{q}_{v_{\mathbb{Q}}}\right)+\frac{12}{l} \cdot \log \left(\mathfrak{s}_{v_{\mathbb{Q}}}^{\mathbb{Q}}\right)+\frac{20}{3 p_{v_{\mathbb{Q}}}} \cdot \log \left(\mathfrak{s}_{v_{\mathbb{Q}}}^{F_{\text {mod }}}\right)\right\}
\end{aligned}
$$

- where, in the passage to the final displayed inequality, we apply the estimates $\frac{1}{l+1} \leq \frac{1}{l}$ and $\frac{4(l+5)}{l+1} \leq \frac{20}{3}$, both of which are consequences of the fact that $l \geq 5$.

(vi) Next, let $v_{\mathbb{Q}} \in \mathbb{V}_{\mathbb{Q}}^{\text {non }} \backslash \mathbb{V}_{\mathbb{Q}}^{\text {dst }}$. Fix $j,\left\{v_{i}\right\}_{i \in \mathbb{S}_{j+1}^{ \pm}}$as in Step (iv). Write $\underline{v}_{i} \in \underline{\mathbb{V}} \stackrel{\sim}{\rightarrow} \mathbb{V}_{\text {mod }}=\mathbb{V}\left(F_{\text {mod }}\right)$ for the element corresponding to $v_{i}$. We would like to apply Proposition 1.4, (iv), to the present situation, by taking

- "I" to be $\mathbb{S}_{j+1}^{ \pm}$;

- " $k_{i}$ " to be $K_{\underline{v}_{i}}$ [so " $R_{i}$ " will be the ring of integers $\mathcal{O}_{K_{\underline{v}_{i}}}$ of $K_{\underline{v}_{i}}$.

Here, we note that our assumption that $v_{\mathbb{Q}} \in \mathbb{V}_{\mathbb{Q}}^{\text {non }} \backslash \mathbb{V}_{\mathbb{Q}}^{\text {dst }}$ implies that the hypotheses of Proposition 1.4, (iv), are satisfied. Thus, the inclusion " $\phi\left(\left(R_{I}\right)^{\sim}\right) \subseteq\left(R_{I}\right) \sim$ " of Proposition 1.4, (iv), implies that the tensor product of log-shells under consideration contains the "union of possible images of a $\Theta$-pilot object" discussed in Step (iv). That is to say, the indeterminacies (Ind1) and (Ind2) are taken into account by the arbitrary nature of the automorphism " $\phi$ " [cf. Proposition 1.2], while the indeterminacy (Ind3) is taken into account by the fact that we are considering upper bounds [cf. the discussion of Step (x) of the proof of [IUTchIII], Corollary 3.12], together with the fact that the "container of possible images" is precisely equal to the tensor product of log-shells under consideration. Thus, an upper bound on the component of the log-volume under consideration may be obtained by computing an upper bound for the log-volume of the right-hand side " $\left(R_{I}\right)$ " " of the above inclusion. Such an upper bound

$$
\text { "0" }
$$

is given in the final equality of Proposition 1.4, (iv). One may then compute a "weighted average upper bound" and then a "procession-normalized upper bound", as was done in Step (v). The resulting "procession-normalized upper bound" is clearly equal to 0 .

(vii) Next, let $v_{\mathbb{Q}} \in \mathbb{V}_{\mathbb{Q}}^{\text {arc }}$. Fix $j,\left\{v_{i}\right\}_{i \in \mathbb{S}_{j+1}^{ \pm}}$as in Step (iv). Write $\underline{v}_{i} \in$ $\underline{\mathbb{V}} \stackrel{\sim}{\rightarrow} \mathbb{V}_{\text {mod }}=\mathbb{V}\left(F_{\text {mod }}\right)$ for the element corresponding to $v_{i}$. We would like to apply Proposition 1.5, (iii), (iv), to the present situation, by taking

- " $I "$ to be $\mathbb{S}_{j+1}^{ \pm}[$so $|I|=j+1]$;

- " $V$ " to be $\mathbb{V}\left(F_{\bmod }\right)_{v_{\mathbb{Q}}}$;

- " $\mathbb{C}_{v}$ " to be $K_{\underline{v}}$, where we write $\underline{v} \in \underline{\mathbb{V}} \stackrel{\sim}{\rightarrow} \mathbb{V}_{\text {mod }}$ for the element determined by $v \in V$. 
Then it follows from Proposition 1.5, (iii), (iv), that

$$
\pi^{j+1} \cdot B_{I}
$$

serves as a container for the "union of possible images of a $\Theta$-pilot object" discussed in Step (iv). That is to say, the indeterminacies (Ind1) and (Ind2) are taken into account by the fact that $B_{I} \subseteq M_{I}$ is preserved by arbitrary automorphisms of the type discussed in Proposition 1.5, (iii), while the indeterminacy (Ind3) is taken into account by the fact that we are considering upper bounds [cf. the discussion of Step (x) of the proof of [IUTchIII], Corollary 3.12], together with the fact that, by Proposition 1.5, (iv), together with our choice of the factor $\pi^{j+1}$, this "container of possible images" contains the elements of $M_{I}$ obtained by forming the tensor product of elements of the log-shells under consideration. Thus, an upper bound on the component of the log-volume under consideration may be obtained by computing an upper bound for the log-volume of this container. Such an upper bound

$$
(j+1) \cdot \log (\pi)
$$

follows immediately from the fact that [in order to ensure compatibility with arithmetic degrees of arithmetic line bundles - cf. [IUTchIII], Proposition 3.9, (iii) one is obliged to adopt normalizations which imply that] the log-volume of $B_{I}$ is equal to 0 . One may then compute a "weighted average upper bound" and then a "procession-normalized upper bound", as was done in Step (v). The resulting "procession-normalized upper bound" is given by

$$
\frac{l+5}{4} \cdot \log (\pi) \leq \frac{l+1}{4} \cdot 4
$$

- cf. (E1), (E6); the fact that $l \geq 5$.

(viii) Now we return to the discussion of Step (iv). In order to compute the desired upper bound for " $C_{\Theta}$ ", it suffices to sum over $v_{\mathbb{Q}} \in \mathbb{V}_{\mathbb{Q}}$ the various local "procession-normalized upper bounds" obtained in Steps (v), (vi), (vii) for $v_{\mathbb{Q}} \in \mathbb{V}_{\mathbb{Q}}$. By applying the inequality of the final display of Step (iii), we thus obtain the following upper bound for " $C_{\Theta}$ ":

$$
\begin{aligned}
\frac{l+1}{4} \cdot\left\{\left(1+\frac{28 \cdot d_{\mathrm{mod}}}{l}\right) \cdot\left(\log \left(\mathfrak{d}^{F_{\mathrm{tpd}}}\right)+\log \left(\mathfrak{f}^{F_{\mathrm{tpd}}}\right)\right)\right. & +2 \cdot \log (l)+69-\frac{1}{6} \cdot \log (\mathfrak{q}) \\
& \left.+\frac{20}{3} \cdot \sum_{v_{\mathbb{Q}} \in \mathbb{V}_{\mathbb{Q}}^{\text {dst }}} \frac{1}{p_{v_{\mathbb{Q}}}} \cdot \log \left(\mathfrak{s}_{v_{\mathbb{Q}}}^{F_{\text {mod }}}\right)\right\}-1
\end{aligned}
$$

- where we apply the estimate $\frac{l+1}{4} \geq 1$ [a consequence of the fact that $l \geq 5$ ]. Thus, it remains to evaluate the sum over $v_{\mathbb{Q}} \in \mathbb{V}_{\mathbb{Q}}^{\text {dst }}$ that appears in the last display. Recall the constant " $\eta_{\text {prm }}$ " of Proposition 1.6. Now we define

$$
\epsilon_{\Theta} \stackrel{\text { def }}{=} \min \left(\frac{40 \eta_{\mathrm{prm}}}{3}, 3^{-210}\right)
$$

and suppose that $\epsilon \in \mathbb{R}_{>0}$ satisfies $\epsilon<\epsilon_{\Theta}$. Note that this implies that $\frac{40}{3 \epsilon}>5$ and $\frac{2}{3} \cdot \log \left(\epsilon^{-1}\right) \geq 140[\mathrm{cf} .(\mathrm{E} 6)]$. Write 


$$
\begin{array}{ll}
\cdot \mathbb{V}_{\mathbb{Q}}^{\prec \epsilon^{-1}} \stackrel{\text { def }}{=}\left\{v_{\mathbb{Q}} \in \mathbb{V}_{\mathbb{Q}} \mid p_{v_{\mathbb{Q}}} \leq \frac{40}{3 \epsilon}\right\} ; \\
\cdot \mathbb{V}_{\mathbb{Q}}^{\succ \epsilon^{-1}} \stackrel{\stackrel{\text { def }}{=}}{=}\left\{v_{\mathbb{Q}} \in \mathbb{V}_{\mathbb{Q}}^{\text {dst }} \mid l \neq p_{v_{\mathbb{Q}}} \geq \frac{40}{3 \epsilon}(>5)\right\} .
\end{array}
$$

Then by applying Proposition 1.6, where we take " $\eta$ " to be $\frac{3 \epsilon}{40}<\eta_{\text {prm }}$, together with the discussion of Step (iii), we conclude that

$$
\begin{aligned}
\frac{20}{3} \cdot \sum_{v_{\mathbb{Q}} \in \mathbb{V}_{\mathbb{Q}}^{\mathrm{dst}}} \frac{1}{p_{v_{\mathbb{Q}}}} \cdot \log \left(\mathfrak{s}_{v_{\mathbb{Q}}^{\text {mod }}}^{F_{\text {mod }}}\right) \\
\leq \frac{20}{3} \cdot \sum_{l=p_{v_{\mathbb{Q}}}} \frac{\log \left(\mathfrak{s}_{v_{\mathbb{Q}}}^{F_{\text {mod }}}\right)}{p_{v_{\mathbb{Q}}}}+\frac{20}{3} \cdot \sum_{v_{\mathbb{Q}} \in \mathbb{V}_{\mathbb{Q}}^{\succ \epsilon}} \frac{\log \left(\mathfrak{s}_{v_{\mathbb{Q}}}^{F_{\text {mod }}}\right)}{p_{v_{\mathbb{Q}}}}+\frac{20}{3} \cdot \sum_{v_{\mathbb{Q}} \in \mathbb{V}_{\mathbb{Q}}^{\prec \epsilon}-1} \frac{\log \left(\mathfrak{s}_{v_{\mathbb{Q}}}^{F_{\text {mod }}}\right)}{p_{v_{\mathbb{Q}}}} \\
\quad \leq 4+\frac{40}{3} \cdot \sum_{v_{\mathbb{Q}} \in \mathbb{V}_{\mathbb{Q}}^{\succ \epsilon}} \frac{1}{p_{v_{\mathbb{Q}}}} \cdot\left(\log \left(\mathfrak{d}_{v_{\mathbb{Q}}}^{F_{\mathrm{tpd}}}\right)+\log \left(\mathfrak{f}_{v_{\mathbb{Q}}}^{F_{\mathrm{tpd}}}\right)\right)+\frac{20}{3} \cdot \sum_{v_{\mathbb{Q}} \in \mathbb{V}_{\mathbb{Q}}^{\prec \epsilon-1}} \frac{\log \left(p_{v_{\mathbb{Q}}}\right)}{p_{v_{\mathbb{Q}}}} \\
\leq 4+\epsilon \cdot\left(\log \left(\mathfrak{d}^{F_{\mathrm{tpd}}}\right)+\log \left(\mathfrak{f}^{F_{\mathrm{tpd}}}\right)\right)-\frac{40}{3} \cdot \log (\epsilon)+\frac{40}{3} \cdot \log \left(\frac{40}{3}\right) \\
\leq \epsilon \cdot\left(\log \left(\mathfrak{d}^{F_{\mathrm{tpd}}}\right)+\log \left(\mathfrak{f}^{F_{\mathrm{tpd}}}\right)\right)+2 \cdot \log \left(\epsilon^{-7}\right)-\frac{2}{3} \cdot \log \left(\epsilon^{-1}\right)+60 \\
\leq \epsilon \cdot\left(\log \left(\mathfrak{d}^{F_{\mathrm{tpd}}}\right)+\log \left(\mathfrak{f}^{F_{\mathrm{tpd}}}\right)\right)+2 \cdot \log \left(\epsilon^{-7}\right)-80
\end{aligned}
$$

— where we apply the estimates $\frac{10}{3} \leq 4$ and $\frac{40}{3} \cdot \log \left(\frac{40}{3}\right) \leq 14 \cdot \log (15) \leq 56$ [cf. (E6)]. Thus, substituting back into our original upper bound for " $C_{\Theta}$ ", we obtain the following upper bound for " $C_{\Theta}$ ":

$$
\frac{l+1}{4} \cdot\left\{\left(1+\epsilon+\frac{28 \cdot d_{\mathrm{mod}}}{l}\right) \cdot\left(\log \left(\mathfrak{d}^{F_{\mathrm{tpd}}}\right)+\log \left(\mathfrak{f}^{F_{\mathrm{tpd}}}\right)\right)+2 \cdot \log \left(l \cdot \epsilon^{-7}\right)-\frac{1}{6} \cdot \log (\mathfrak{q})\right\}-1
$$

- where we apply the estimate $69-80 \leq 0$ - i.e., as asserted in the statement of Theorem 1.10. The final portion of Theorem 1.10 follows immediately from [IUTchIII], Corollary 3.12, together with the inequality of the first display of Step (ii).

Remark 1.10.1. One of the main original motivations for the development of the theory discussed in the present series of papers was to create a framework, or geometry, within which a suitable analogue of the scheme-theoretic Hodge-Arakelov theory of [HASurI], [HASurII] could be realized in such a way that the obstructions to diophantine applications that arose in the scheme-theoretic formulation of [HASurI], [HASurII] [cf. the discussion of [HASurI], §1.5.1; [HASurII], Remark 3.7] could be avoided. From this point of view, it is of interest to observe that the computation of the "leading term" of the inequality of the final display of the statement of Theorem 1.10 - i.e., of the term

$$
\frac{\left(l^{*}+3\right)}{2} \cdot \log \left(\mathfrak{d}_{v_{\mathbb{Q}}}^{K}\right)-\frac{\left(2 l^{*}+1\right)\left(l^{*}+1\right)}{12 l} \cdot \log \left(\mathfrak{q}_{v_{\mathbb{Q}}}\right)
$$

that occurs in the final display of Step (v) of the proof of Theorem 1.10 — via the identities (E1), (E2) is essentially identical to the computation of the leading term that occurs in the proof of [HASurI], Theorem A [cf. the discussion following the statement of Theorem A in [HASurI], §1.1]. That is to say, in some sense, 
the computations performed in the proof of Theorem 1.10 were already essentially known to the author around the year 2000; the problem then was to construct an appropriate framework, or geometry, in which these computations could be performed!

This sort of situation may be compared to the computations underlying the Weil Conjectures priori to the construction of a "Weil cohomology" in which those computations could be performed, or, alternatively, to various computations of invariants in topology or differential geometry that were motivated by computations in physics, again prior to the construction of a suitable mathematical framework in which those computations could be performed.

Remark 1.10.2. The computation performed in the proof of Theorem 1.10 may be thought of as the computation of a sort of derivative in the $\mathbb{F}_{l}^{*}$-direction, which, relative to the analogy between the theory of the present series of papers and the $p$-adic Teichmüller theory of $[p$ Ord], $[p$ Teich], corresponds to the derivative of the canonical Frobenius lifting — cf. the discussion of [IUTchIII], Remark 3.12.4, (iii). In this context, it is useful to recall the arithmetic Kodaira-Spencer morphism that occurs in scheme-theoretic Hodge-Arakelov theory [cf. [HASurII], §3]. In particular, in [HASurII], Corollary 3.6, it is shown that, when suitably formulated, a "certain portion" of this arithmetic Kodaira-Spencer morphism coincides with the usual geometric Kodaira-Spencer morphism. From the point of view of the action of $G L_{2}\left(\mathbb{F}_{l}\right)$ on the l-torsion points involved, this "certain portion" consists of the unipotent matrices

$$
\left(\begin{array}{ll}
1 & * \\
0 & 1
\end{array}\right)
$$

of $G L_{2}\left(\mathbb{F}_{l}\right)$. By contrast, the $\mathbb{F}_{l}^{*}$-symmetries that occur in the present series of papers correspond to the toral matrices

$$
\left(\begin{array}{cc}
1 & 0 \\
0 & \pm *
\end{array}\right)
$$

of $G L_{2}\left(\mathbb{F}_{l}\right)$ - cf. the discussion of [IUTchI], Example 4.3, (i). As we shall see in $\S 2$ below, in the present series of papers, we shall ultimately take $l$ to be "large". When $l$ is "sufficiently large", $G L_{2}\left(\mathbb{F}_{l}\right)$ may be thought of as a "good approximation" for $G L_{2}(\mathbb{Z})$ or $G L_{2}(\mathbb{R})$ - cf. the discussion of [IUTchI], Remark 6.12.3, (i), (iii). In the case of $G L_{2}(\mathbb{R})$, "toral subgroups" may thought of as corresponding to the isotropy subgroups [isomorphic to $\mathbb{S}^{1}$ ] of points that arise from the action of $G L_{2}(\mathbb{R})$ on the upper half-plane, i.e., subgroups which may be thought of as a sort of geometric, group-theoretic representation of tangent vectors at a point.

Remark 1.10.3. The " $\log (l)$ term" that occurs in the inequality of the final display of Theorem 1.10 may be thought of as an inevitable consequence of the fundamental role played in the theory of the present series of papers by the $l$-torsion points of the elliptic curve under consideration. Here, we note that it is of crucial importance to work over the field of rationality of the l-torsion points [i.e., " $K$ " as opposed to " $F$ "] not only when considering the global portions of the various $\Theta N F$ - 
and $\Theta^{ \pm \text {ell }}$-Hodge-theaters involved, but also when considering the local portions i.e., the prime-strips - of these $\Theta \mathrm{NF}$ - and $\Theta^{ \pm \text {ell }}$-Hodge-theaters. That is to say, these local portions are necessary, for instance, in order to glue together the $\Theta \mathrm{NF}$ and $\Theta^{ \pm \text {ell }}$-Hodge-theaters that appear so as to form a $\Theta^{ \pm \text {ell }} N F$-Hodge-theater [cf. the discussion of [IUTchI], Remark 6.12.2]. In particular, to allow, within these local portions, any sort of "Galois indeterminacy" with respect to the $l$-torsion points - even, for instance, at $\underline{v} \in \underline{\mathbb{V}}^{\text {good }} \cap \underline{\mathbb{V}}^{\text {non }}$, which, at first glance, might appear irrelevant to the theory of Hodge-Arakelov-theoretic evaluation at l-torsion points developed in [IUTchII] — would have the effect of invalidating the various delicate manipulations involving l-torsion points discussed in [IUTchI], §4, §6 [cf., e.g., [IUTchI], Propositions 4.7, 6.5].

Remark 1.10.4. The various fluctuations in log-volume - i.e., whose computation is the subject of Theorem 1.10! — that arise from the multiradial representation of [IUTchIII], Theorem 3.11, (i), may be thought of as a sort of "inter-universal analytic torsion". Indeed,

in general, "analytic torsion" may be understood as a sort of measure in "metrized" [e.g., log-volume!] terms — of the degree of deviation of the "holomorphic functions" [such as sections of a line bundle] on a variety - i.e., which depend, in an essential way, on the holomorphic moduli of the variety! — from the "real analytic functions" - i.e., which are invariant with respect to deformations of the holomorphic moduli of the variety.

For instance:

(a) In "classical" Arakelov theory, analytic torsion typically arises as [the logarithm of] a sort of normalized determinant of the Laplacian acting on some space of real analytic [or $\mathrm{L}^{2}$-] sections of a line bundle on a complex variety equipped with a real analytic Kähler metric [cf., e.g., [Arak], Chapters V, VI]. Here, we recall that in this sort of situation, the space of holomorphic sections of the line bundle is given by the kernel of the Laplacian; the definition of the Laplacian depends, in an essential way, on the Kähler metric, hence, in particular, on the holomorphic moduli of the variety under consideration [cf., e.g., the case of the Poincaré metric on a hyperbolic Riemann surface!].

(b) In the scheme-theoretic Hodge-Arakelov theory discussed in [HASurI], [HASurII], the main theorem consists of a sort of comparison isomorphism [cf. [HASurI], Theorem A] between the space of sections of an ample line bundle on the universal vectorial extension of an elliptic curve and the space of set-theoretic functions on the torsion points of the elliptic curve. That is to say, the former space of sections contains, in a natural way, the space of holomorphic sections of the ample line on the elliptic curve, while the latter space of functions may be thought of as a sort of "discrete approximation" of the space of real analytic functions on the elliptic curve [cf. the discussion of [HASurI], §1.3.2, §1.3.4]. In this context, the "Gaussian poles" [cf. the discussion of [HASurI], §1.1] arise as a measure of the discrepancy of integral structures between these two spaces in a neighborhood of the divisor at 
infinity of the moduli stack of elliptic curves, hence may be thought of as a sort of "analytic torsion at the divisor at infinity" [cf. the discussion of [HASurI], §1.2].

(c) In the case of the multiradial representation of [IUTchIII], Theorem 3.11, (i), the fluctuations of log-volume computed in Theorem 1.10 arise precisely as a result of the execution of a comparison of an "alien" arithmetic holomorphic structure to this multiradial representation, which is compatible with the permutation symmetries of the étale-picture, i.e., which is "invariant with respect to deformations of the arithmetic holomorphic moduli of the number field under consideration" in the sense that it makes sense simultaneously with respect to distinct arithmetic holomorphic structures [cf. [IUTchIII], Remark 3.11.1; [IUTchIII], Remark 3.12.3, (ii)]. Here, it is of interest to observe that the object of this comparison consists of the values of the theta function, i.e., in essence, a "holomorphic section of an ample line bundle". In particular, the resulting fluctuations of log-volume may be thought as a sort of "analytic torsion". By analogy to the terminology "Gaussian poles" discussed in (b) above, it is natural to think of the terms involving the different $\mathfrak{d}_{(-)}^{K}$ that appear in the computation underlying Theorem 1.10 [cf., e.g., the final display of Step (v) of the proof of Theorem 1.10] as "differential poles" [cf. the discussion of Remarks 1.10.1, 1.10.2]. Finally, in the context of the normalized determinants that appear in (a), it is interesting to note the role played by the prime number theorem - i.e., in essence, the Riemann zeta function [cf. Proposition 1.6 and its proof] — in the computation of "inter-universal analytic torsion" given in the proof of Theorem 1.10. 


\section{Section 2: Diophantine Inequalities}

In the present $\S 2$, we combine Theorem 1.10 with the theory of [GenEll] to give a proof of the ABC Conjecture, or, equivalently, Vojta's Conjecture for hyperbolic curves [cf. Corollary 2.3 below].

We begin by reviewing some well-known estimates.

\section{Proposition 2.1. (Well-known Estimates)}

(i) (Linearization of Logarithms) Let $C, \epsilon \in \mathbb{R}_{>0}$. Then there exists an $x_{0} \in \mathbb{R}_{>0}$ such that $\log (x+C) \leq \epsilon \cdot x$ for all $(\mathbb{R} \ni) x \geq x_{0}$.

(ii) (The Prime Number Theorem) There exists a $\xi_{\mathrm{prm}} \in \mathbb{R}_{>0}$ such that

$$
\sum_{p \leq x} \log (p) \leq 2 x
$$

- where the sum ranges over the prime numbers $p$ such that $p \leq x$ - for all $(\mathbb{R} \ni) x \geq \xi_{\text {prm }}$.

Proof. Assertion (i) is well-known and entirely elementary. Assertion (ii) is a well-known consequence of the Prime Number Theorem [cf., e.g., [Edw], p. 76].

Let $\overline{\mathbb{Q}}$ be an algebraic closure of $\mathbb{Q}$. In the following discussion, we shall apply the notation and terminology of [GenEll]. Let $X$ be a smooth, proper, geometrically connected curve over a number field; $D \subseteq X$ a reduced divisor; $U_{X} \stackrel{\text { def }}{=} X \backslash D$; $d$ a positive integer. Write $\omega_{X}$ for the canonical sheaf on $X$. Suppose that $U_{X}$ is a hyperbolic curve, i.e., that the degree of the line bundle $\omega_{X}(D)$ is positive. Then we recall the following notation:

- $U_{X}(\overline{\mathbb{Q}}) \leq d \subseteq U_{X}(\overline{\mathbb{Q}})$ denotes the subset of $\overline{\mathbb{Q}}$-rational points defined over a finite extension field of $\mathbb{Q}$ of degree $\leq d$ [cf. [GenEll], Example 1.3, (i)].

- log-diff ${ }_{X}$ denotes the (normalized) log-different function on $U_{X}(\overline{\mathbb{Q}}) \leq d[\mathrm{cf}$. [GenEll], Definition 1.5, (iii)].

- $\log$-cond ${ }_{D}$ denotes the (normalized) log-conductor function on $U_{X}(\overline{\mathbb{Q}}) \leq d$ [cf. [GenEll], Definition 1.5, (iv)].

- ht $_{\omega_{X}(D)}$ denotes the (normalized) height function on $U_{X}(\overline{\mathbb{Q}}) \leq d$ associated to $\omega_{X}(D)$, which is well-defined up to a "bounded discrepancy" [cf. [GenEll], Proposition 1.4, (iii)];

In order to apply the theory of the present series of papers, it is neceesary to construct suitable initial $\Theta$-data, as follows. 
Corollary 2.2. (Construction of Suitable Initial $\Theta$-Data) Suppose that $X=\mathbb{P}_{\mathbb{Q}}^{1}$ is the projective line over $\mathbb{Q}$, and that $D \subseteq X$ is the divisor consisting of the three points " 0 ", " 1 ", and " $\infty$ ". We shall regard $X$ as the " $\lambda$-line" - i.e., we shall regard the standard coordinate on $X=\mathbb{P}_{\mathbb{Q}}^{1}$ as the " $\lambda$ " in the Legendre form " $y^{2}=x(x-1)(x-\lambda)$ " of the Weierstrass equation defining an elliptic curve and hence as being equipped with a natural classifying morphism $U_{X} \rightarrow\left(\mathcal{M}_{\mathrm{ell}}\right)_{\mathbb{Q}}$ [cf. the discussion preceding Proposition 1.8]. Let

$$
\mathcal{K}_{V} \subseteq U_{X}(\overline{\mathbb{Q}})
$$

be a compactly bounded subset [i.e., regarded as a subset of $X(\overline{\mathbb{Q}})-c f$. [GenEll], Example 1.3, (ii)] whose support contains the nonarchimedean prime "2". Then:

(i) The normalized degree "deg(-)" of the effective arithmetic divisor determined by the $\boldsymbol{q}$-parameters of an elliptic curve over a number field at the nonarchimedean primes that do not divide 2 [cf. the invariant $\log (\mathfrak{q})$ " associated, in the statement of Theorem 1.10, to the elliptic curve $\left.E_{F}\right]$ determines an $\mathbb{R}$-valued function on $\mathcal{M}_{\mathrm{ell}}(\overline{\mathbb{Q}})$, hence also on $U_{X}(\overline{\mathbb{Q}})$. If we denote this function by the notation $" \log \left(\mathfrak{q}_{(-)}\right)$", then we have an equality of "bounded discrepancy classes" [cf. [GenEll], Definition 1.2, (ii), as well as Remark 2.3.1, (ii), below]

$$
\frac{1}{6} \cdot \log \left(\mathfrak{q}_{(-)}\right) \approx \operatorname{ht}_{\omega_{X}(D)}
$$

of functions on $\mathcal{K}_{V} \subseteq U_{X}(\overline{\mathbb{Q}})$.

(ii) Let

$$
\mathbb{R}_{>0} \ni \epsilon<\min \left(\xi_{\text {prm }}^{-1}, 5 \cdot \epsilon_{\Theta}\right)
$$

- where $\epsilon_{\Theta}$ is as in Theorem 1.10; $\xi_{\mathrm{prm}}$ is as in Proposition 2.1, (ii). Then there exists a Galois-finite [cf. [GenEll], Example 1.3, (i)] subset $\mathfrak{E x c}_{\epsilon} \subseteq U_{X}(\overline{\mathbb{Q}})$ which contains all points corresponding to elliptic curves with automorphisms of order $>2$ and, moreover, satisfies the following property:

Let $E_{F}$ be an elliptic curve over a number field $F \subseteq \overline{\mathbb{Q}}$ that lifts [not necessarily uniquely!] to a point $x_{E} \in U_{X}(F)$ such that

$$
\left[x_{E}\right] \in \mathcal{K}_{V}, \quad\left[x_{E}\right] \notin \mathfrak{E x c}_{\epsilon} .
$$

Write $F_{\bmod }$ for the minimal field of definition of the corresponding point $\in$ $\mathcal{M}_{\text {ell }}(\overline{\mathbb{Q}})$ and

$$
F_{\text {mod }} \subseteq F_{\text {tpd }} \stackrel{\text { def }}{=} F_{\text {mod }}\left(E_{F_{\text {mod }}}[2]\right) \subseteq F
$$

for the "tripodal" intermediate field obtained from $F_{\bmod }$ by adjoining the fields of definition of the 2-torsion points of any model of $E_{F}$ over $F_{\bmod }$ [cf. Proposition 1.8, (ii), (iii)]. Moreover, we assume that the $(3 \cdot 5)$-torsion points of $E_{F}$ are defined over $F$, and that

$$
F=F_{\text {mod }}\left(\sqrt{-1}, E_{F_{\text {mod }}}[2 \cdot 3 \cdot 5]\right) \stackrel{\text { def }}{=} F_{\mathrm{tpd}}\left(\sqrt{-1}, E_{F_{\mathrm{tpd}}}[3 \cdot 5]\right)
$$


- i.e., that $F$ is obtained from $F_{\mathrm{tpd}}$ by adjoining $\sqrt{-1}$, together with the fields of definition of the $(3 \cdot 5)$-torsion points of a model $E_{F_{\mathrm{tpd}}}$ of the elliptic curve $E_{F}$ over $F_{\text {tpd }}$ determined by the Legendre form of the Weierstrass equation discussed above [cf. Proposition 1.8, (vi)]. [Thus, it follows from Proposition 1.8, (iv), that $E_{F} \cong E_{F_{\mathrm{tpd}}} \times_{F_{\mathrm{tpd}}} F$ over $F$, so $x_{E} \in U_{X}\left(F_{\mathrm{tpd}}\right) \subseteq U_{X}(F)$.] Then $E_{F}$ and $F_{\mathrm{mod}}$ arise as the " $E_{F}$ " and " $F_{\text {mod }}$ " for a collection of initial $\Theta$-data as in Theorem 1.10 such that, in the notation of Theorem 1.10, the prime number $l$ satisfies the following conditions:

(P1) $2 \cdot \log (l) \leq \frac{1}{5} \cdot \epsilon \cdot\left\{\frac{1}{6} \cdot \log (\mathfrak{q})+\log \left(\mathfrak{d}^{F_{\mathrm{tpd}}}\right)\right\}+2 \cdot \log \left(d_{\mathrm{mod}}\right) ;$

(P2) $l \geq 5 \cdot 28 \cdot d_{\mathrm{mod}} \cdot \epsilon^{-1}$.

In particular, by applying Theorem 1.10, we conclude that

$\frac{1}{6} \cdot \log (\mathfrak{q}) \leq(1+\epsilon) \cdot\left(\log -\operatorname{diff}_{X}\left(x_{E}\right)+\log -\operatorname{cond}_{X}\left(x_{E}\right)\right)+28 \cdot \log \left(5 \cdot \epsilon^{-1}\right)+4 \cdot \log \left(d_{\bmod }\right)$

- where we observe that [it follows tautologically from the definitions that] we have: $\log$-diff $X_{X}\left(x_{E}\right)=\log \left(\mathfrak{d}^{F_{\mathrm{tpd}}}\right), \log -\operatorname{cond}_{D}\left(x_{E}\right)=\log \left(\mathfrak{f}^{F_{\mathrm{tpd}}}\right)$.

Proof. First, we consider assertion (i). We begin by observing that since the support of $\mathcal{K}_{V}$ contains the nonarchimedean prime "2", it follows immediately from the various definitions involved that

$$
\log \left(\mathfrak{q}_{(-)}\right) \approx \underline{\operatorname{deg}}_{\infty}
$$

- where "deg ${ }_{\infty}$ " is as in the discussion preceding [GenEll], Proposition 3.4 on $\mathcal{K}_{V} \subseteq U_{X}(\overline{\mathbb{Q}})$. In a similar vein, since the support of $\mathcal{K}_{V}$ contains the unique archimedean prime of $\mathbb{Q}$, it follows immediately from the various definitions involved that

$$
\underline{\operatorname{deg}}_{\infty} \approx \mathrm{ht}_{\infty}
$$

- where "ht ${ }_{\infty}$ " is as in the discussion preceding [GenEll], Proposition 3.4 - on $\mathcal{K}_{V} \subseteq U_{X}(\overline{\mathbb{Q}})$ [cf. the argument of the final paragraph of the proof of [GenEll], Lemma 3.7]. Thus, we conclude that $\log \left(\mathfrak{q}_{(-)}\right) \approx h t_{\infty}$. Since [as is well-known] the pull-back to $X$ of the divisor at infinity of the natural compatification $\left(\overline{\mathcal{M}}_{\text {ell }}\right)_{\mathbb{Q}}$ of $\left(\mathcal{M}_{\text {ell }}\right)_{\mathbb{Q}}$ is of degree 6 , while the line bundle $\omega_{X}(D)$ is of degree 1 , the desired equality of BD-classes $\frac{1}{6} \cdot \log \left(\mathfrak{q}_{(-)}\right) \approx \mathrm{ht}_{\omega_{X}(D)}$ follows immediately from [GenEll], Proposition 1.4, (i), (iii). This completes the proof of assertion (i).

Next, we consider assertion (ii). First, we observe that [one verifies easily that] the image in $\mathcal{M}_{\text {ell }}(\overline{\mathbb{Q}})$ of $\mathcal{K}_{V}$ determines a compactly bounded subset of $\mathcal{M}_{\text {ell }}(\overline{\mathbb{Q}})$. Thus, by applying [GenEll], Corollary 4.4, to this compactly bounded subset of $\mathcal{M}_{\text {ell }}(\overline{\mathbb{Q}})$, we obtain a Galois-finite subset "ecc" of $\mathcal{M}_{\text {ell }}(\overline{\mathbb{Q}})$, together with a constant "C $\in \mathbb{R}$ ", that satisfy a certain property [cf. the statement of [GenEll], Corollary 4.4], which we shall discuss below in detail. Let us write

$$
\mathfrak{E x c}_{\epsilon} \subseteq U_{X}(\overline{\mathbb{Q}})
$$

for the inverse image of the subset "Exc" of [GenEll], Corollary 4.4, and $C_{1}$ for the constant " $C$ ". One verifies immediately that this subset $\mathfrak{E x c} \in \subseteq U_{X}(\overline{\mathbb{Q}})$ is 
Galois-finite. Although $\mathfrak{E x c}_{\epsilon}$, defined in this way, does not depend on $\epsilon$, we shall, in the argument to follow, enlarge $\mathfrak{E x} \mathfrak{c}_{\epsilon}$ several times - i.e., by an abuse of notation, for the purpose of simplifying the notation! - in such a way that the resulting enlargement does in fact depend on $\epsilon$.

Next, let us recall that if the once-punctured elliptic curve associated to $E_{F}$ fails to admit an $F$-core, then there are only four possibilities for the $j$-invariant of $E_{F}$ [cf. [CanLift], Proposition 2.7]. Thus, by possibly enlarging $\mathfrak{E x c}_{\epsilon}$ [in a fashion that is still independent of $\epsilon !$, which is possible in light of [GenEll], Proposition 1.4, (iv), we may assume that the once-punctured elliptic curve associated to $E_{F}$ admits an $F$-core, hence, in particular, does not have any automorphisms of order $>2$ over $\overline{\mathbb{Q}}$.

Now before proceeding, let us observe [cf., Proposition 1.8, (v)] that our assumptions concerning the extension $F / F_{\text {mod }}$ imply that $E_{F}$ has stable reduction at all of the nonarchimedean primes of $F$ [cf. the proof of [GenEll], Theorem 3.8]. Next, let us observe that it follows from assertion (i) that the function "ht $\omega_{\omega_{X}}(D)$ " is bounded on the set of $\left[x_{E}\right]$ corresponding to $E_{F}$ with good reduction at all nonarchimedean primes that do not divide 2. In particular, by possibly enlarging $\mathfrak{E x c}_{\epsilon}$ [in a fashion that is still independent of $\epsilon !$ ], which is possible in light of [GenEll], Proposition 1.4, (iv), we may assume that $E_{F}$ has bad [but stable!] reduction at some nonarchimedean prime that does not divide 2. Thus, in summary, one verifies immediately [cf., especially, our assumptions concerning the extension $F / F_{\text {mod }}$ ] that all of the conditions of [IUTchI], Definition 3.1, (a), (b), (d), (e), (f), are satisfied. That is to say, in order to obtain a collection of initial $\Theta$-data as in the statement of assertion (ii), it suffices to show the existence of a prime number $l$ that satisfies the conditions of [IUTchI], Definition 3.1, (c), as well as the conditions (P1), (P2) of the statement of assertion (ii).

Next, we would like to apply the property satisfied by the subset "Exc" of [GenEll], Corollary 4.4. We take the set "S $\mathcal{S}$ " of loc. cit. to be the set

$$
\mathcal{S} \stackrel{\text { def }}{=}\left\{p \mid p \text { is a prime number } \leq 5 \cdot 28 \cdot d_{\text {mod }} \cdot \epsilon^{-1}(>5)\right\}
$$

— cf. condition (P2). Thus, we obtain the estimate

$$
x_{\mathcal{S}} \stackrel{\text { def }}{=} \sum_{p \in \mathcal{S}} \log (p) \leq 10 \cdot 28 \cdot d_{\bmod } \cdot \epsilon^{-1}
$$

- cf. our assumption that $\epsilon^{-1} \geq \xi_{\text {prm }}$; Proposition 2.1, (ii). Note that the quantity " $d$ " of loc. cit. corresponds to the quantity $d_{\text {mod }}$ of the present discussion. Now we take the prime number $l$ to be the prime number "l•" of [GenEll], Corollary 4.4. Thus, $l \notin \mathcal{S}$, so the condition (P2) is satisfied. Moreover, since $2,3,5 \in \mathcal{S}$, it follows from conditions (a), (b) of [GenEll], Corollary 4.4, that the conditions of [IUTchI], Definition 3.1, (c), are satisfied.

Next, let us observe that it follows from the argument applied in the proof of assertion (i), together with [GenEll], Proposition 3.4, that we have equalities/inequalities of BD-classes

$$
\log \left(\mathfrak{q}_{(-)}\right) \approx \mathrm{ht}_{\infty} \gtrsim 12 \cdot \mathrm{ht}^{\text {Falt }}
$$


on $\mathcal{K}_{V} \subseteq U_{X}(\overline{\mathbb{Q}})$. Thus, it follows from condition (c) of [GenEll], Corollary 4.4, that

$$
\begin{aligned}
l & \leq d_{\text {mod }} \cdot\left\{23040 \cdot 50 \cdot \frac{1}{6} \cdot \log (\mathfrak{q})+6 \cdot \log \left(\mathfrak{d}^{F_{\text {mod }}}\right)+2 x_{\mathcal{S}} \cdot d_{\text {mod }}^{-1}+C_{2}\right\} \\
& \leq d_{\text {mod }} \cdot\left\{23040 \cdot 50 \cdot \frac{1}{6} \cdot \log (\mathfrak{q})+6 \cdot \log \left(\mathfrak{d}^{F_{\text {mod }}}\right)+20 \cdot 28 \cdot \epsilon^{-1}+C_{2}\right\} \\
& \leq d_{\text {mod }} \cdot\left\{23040 \cdot 50 \cdot \frac{1}{6} \cdot \log (\mathfrak{q})+6 \cdot \log \left(\mathfrak{d}^{F_{\text {tpd }}}\right)+C_{\epsilon}\right\}
\end{aligned}
$$

- where, in the first inequality, we replace the constant $C_{1}$ by a new constant $C_{2}$, so as to take into account the inequality of BD-classes discussed above; in the third inequality, we take $C_{\epsilon} \stackrel{\text { def }}{=} 20 \cdot 28 \cdot \epsilon^{-1}+C_{2}$; we observe that the quantity "log-diff $\overline{\mathcal{M}}_{\text {ell }}\left(\left[E_{L}\right]\right)$ " of loc. cit. [cf. Remark 2.3.1, (iv), below] corresponds to the quantity $\log \left(\mathfrak{d}^{F_{\text {mod }}}\right) \quad\left(\leq \log \left(\mathfrak{d}^{F_{\mathrm{tpd}}}\right)\right)$ of the present discussion. Next, let us observe that since $\log \left(\mathfrak{d}^{F_{\mathrm{tpd}}}\right) \geq 0$ and $C_{\epsilon}=20 \cdot 28 \cdot \epsilon^{-1}+C_{2} \geq C_{2}$, it follows that any upper bound on the quantity

$$
\left\{23040 \cdot 50 \cdot \frac{1}{6} \cdot \log (\mathfrak{q})+6 \cdot \log \left(\mathfrak{d}^{F_{\mathrm{tpd}}}\right)+C_{\epsilon}\right\}
$$

of the final line of the preceding display implies an upper bound on the quantity $\log (\mathfrak{q})$, i.e., [by applying the equalities of BD-classes discussed above] an upper bound on the quantity "ht ${ }_{\infty}$ ", which [cf., e.g., [GenEll], Proposition 1.4, (iv)] can only be satisfied by finitely many elements of $\mathcal{M}_{\text {ell }}(\overline{\mathbb{Q}})^{\leq n}$, for a given integer $n$. Thus, by possibly enlarging $\mathfrak{E x c}_{\epsilon}$ [this time in a way that depends on $\epsilon$ !], we may assume, by applying Proposition 2.1, (i), that

$$
2 \cdot \log (l) \leq \frac{1}{5} \cdot \epsilon \cdot\left\{\frac{1}{6} \cdot \log (\mathfrak{q})+\log \left(\mathfrak{d}^{F_{\mathrm{tpd}}}\right)\right\}+2 \cdot \log \left(d_{\mathrm{mod}}\right)
$$

- i.e., that the condition (P1) is satisfied.

Finally, we observe that since, by assumption, $\frac{1}{5} \cdot \epsilon<\epsilon_{\Theta}$, it follows from the final portion of Theorem 1.10 that

$$
\begin{aligned}
\frac{1}{6} \cdot \log (\mathfrak{q}) \leq & \left(1+\frac{1}{5} \cdot \epsilon+\frac{28 \cdot d_{\mathrm{mod}}}{l}\right) \cdot\left(\log \left(\mathfrak{d}^{F_{\mathrm{tpd}}}\right)+\log \left(\mathfrak{f}^{F_{\mathrm{tpd}}}\right)\right) \\
& +2 \cdot \log (l)+14 \cdot \log \left(5 \cdot \epsilon^{-1}\right) \\
\leq & \left(1+\frac{1}{5} \cdot \epsilon+\frac{1}{5} \cdot \epsilon\right) \cdot\left(\log \left(\mathfrak{d}^{F_{\mathrm{tpd}}}\right)+\log \left(\mathfrak{f}^{F_{\mathrm{tpd}}}\right)\right) \\
& +\frac{1}{5} \cdot \epsilon \cdot\left\{\frac{1}{6} \cdot \log (\mathfrak{q})+\log \left(\mathfrak{d}^{F_{\mathrm{tpd}}}\right)\right\}+14 \cdot \log \left(5 \cdot \epsilon^{-1}\right)+2 \cdot \log \left(d_{\mathrm{mod}}\right) \\
\leq & \frac{1}{5} \cdot \epsilon \cdot \frac{1}{6} \cdot \log (\mathfrak{q})+\left(1+\frac{3}{5} \cdot \epsilon\right) \cdot\left(\log \left(\mathfrak{d}^{F_{\mathrm{tpd}}}\right)+\log \left(\mathfrak{f}^{F_{\mathrm{tpd}}}\right)\right) \\
& +14 \cdot \log \left(5 \cdot \epsilon^{-1}\right)+2 \cdot \log \left(d_{\mathrm{mod}}\right)
\end{aligned}
$$

— where we apply the inequalities of (P1), (P2), as well as the inequality $\log \left(\mathfrak{f}^{F_{\mathrm{tpd}}}\right) \geq$ 0 . The inequality

$\frac{1}{6} \cdot \log (\mathfrak{q}) \leq(1+\epsilon) \cdot\left(\log -\operatorname{diff}_{X}\left(x_{E}\right)+\log -\operatorname{cond}_{D}\left(x_{E}\right)\right)+28 \cdot \log \left(5 \cdot \epsilon^{-1}\right)+4 \cdot \log \left(d_{\text {mod }}\right)$

[cf. the final display of the statement of assertion (ii)] thus follows by applying the estimates

$$
\frac{1+\frac{3}{5} \cdot \epsilon}{1-\frac{1}{5} \cdot \epsilon} \leq 1+\epsilon ; \quad 1-\frac{1}{5} \cdot \epsilon \geq \frac{1}{2}
$$


- both of which are consequences of the fact that $0<\epsilon \leq 1$ - together with the observation that it follows immediately from the definitions [cf. also Proposition $1.8,(\mathrm{vi})]$ that the quantities $\log$ - $\operatorname{diff}_{X}\left(x_{E}\right), \log$-cond ${ }_{D}\left(x_{E}\right)$ correspond precisely to the quantities $\log \left(\mathfrak{d}^{F_{\mathrm{tpd}}}\right), \log \left(\mathfrak{f}^{F_{\mathrm{tpd}}}\right)$, respectively.

We are now ready to state and prove the main theorem of the present $\S 2$, which may also be regarded as the main application of the theory developed in the present series of papers.

Corollary 2.3. (Diophantine Inequalities) Let $X$ be a smooth, proper, geometrically connected curve over a number field; $D \subseteq X$ a reduced divisor; $U_{X} \stackrel{\text { def }}{=}$ $X \backslash D ; d$ a positive integer; $\epsilon \in \mathbb{R}_{>0}$ a positive real number. Write $\omega_{X}$ for the canonical sheaf on $X$. Suppose that $U_{X}$ is a hyperbolic curve, i.e., that the degree of the line bundle $\omega_{X}(D)$ is positive. Then, relative to the notation reviewed above, one has an inequality of "bounded discrepancy classes"

$$
\text { ht }_{\omega_{X}(D)} \lesssim(1+\epsilon)\left(\log -\operatorname{diff}_{X}+\log -\operatorname{cond}_{D}\right)
$$

of functions on $U_{X}(\overline{\mathbb{Q}})^{\leq d}-$ i.e., the function $(1+\epsilon)\left(\log\right.$-diff ${ }_{X}+\log$-cond $\left.{ }_{D}\right)-$ $\mathrm{ht}_{\omega_{X}(D)}$ is bounded below by a constant on $U_{X}(\overline{\mathbb{Q}}) \leq d$ [cf. [GenEll], Definition 1.2, (ii), as well as Remark 2.3.1, (ii), below].

Proof. One verifies immediately that the content of the statement of Corollary 2.3 coincides precisely with the content of [GenEll], Theorem 2.1, (i). Thus, it follows from the equivalence of [GenEll], Theorem 2.1, that, in order to complete the proof of Corollary 2.3, it suffices to verify that Theorem 2.1, (ii), holds. That is to say, we may assume without loss of generality that:

- $X=\mathbb{P}_{\mathbb{Q}}^{1}$ is the projective line over $\mathbb{Q}$;

- $D \subseteq X$ is the divisor consisting of the three points "0", "1", and " $\infty$ ";

- $\mathcal{K}_{V} \subseteq U_{X}(\overline{\mathbb{Q}})$ is a compactly bounded subset whose support contains the nonarchimedean prime "2".

Then it suffices to show that the inequality of BD-classes of functions [cf. [GenEll], Definition 1.2, (ii), as well as Remark 2.3.1, (ii), below]

$$
\mathrm{ht}_{\omega_{X}(D)} \lesssim(1+\epsilon)\left(\log -\operatorname{diff}_{X}+\log -\operatorname{cond}_{D}\right)
$$

holds on $\mathcal{K}_{V} \cap U_{X}(\overline{\mathbb{Q}})^{\leq d}$. But such an inequality follows immediately, in light of the equality of BD-classes of Corollary 2.2, (i), from the final portion of Corollary 2.2 , (ii) [where we note that it follows immediately from the various definitions involved that $\left.d_{\text {mod }} \leq d\right]$. This completes the proof of Corollary 2.3.

Remark 2.3.1. We take this opportunity to correct some unfortunate misprints in [GenEll].

(i) The notation "ord $(-): F_{v} \rightarrow \mathbb{Z}$ " in the final sentence of the first paragraph following [GenEll], Definition 1.1, should read " $\operatorname{ord}_{v}(-): F_{v}^{\times} \rightarrow \mathbb{Z}$ ". 
(ii) In [GenEll], Definition 1.2, (ii), the non-resp'd and first resp'd items in the display should be reversed! That is to say, the notation " $\alpha \lesssim_{\mathcal{F}} \beta$ " corresponds to " $\alpha(x)-\beta(x) \leq C$ "; the notation " $\alpha \gtrsim_{\mathcal{F}} \beta$ " corresponds to " $\beta(x)-\alpha(x) \leq C$ ".

(iii) The first portion of the first sentence of the statement of [GenEll], Corollary 4.4 , should read: "Let $\overline{\mathbb{Q}}$ be an algebraic closure of $\mathbb{Q} ;$...".

(iv) The "log-diff $\left.\overline{\mathcal{M}}_{\text {ell }}\left(\left[E_{L}\right]\right)\right)$ " in the second inequality of the final display of the statement of [GenEll], Corollary 4.4, should read "log-diff $\overline{\mathcal{M}}_{\text {ell }}\left(\left[E_{L}\right]\right)$ ".

\section{Remark 2.3.2.}

(i) The reader will note that, by arguing with a "bit more care", it is not difficult to give stronger estimates for the various "constants" that occur in Theorem 1.10; Corollaries 2.2, 2.3 and their proofs. Such stronger estimates are, however, being the scope of the present series of papers, so we shall not pursue this topic further in the present paper.

(ii) On the other hand, we recall that the constant " 1 " in the inequality of the display of Corollary 2.3 cannot be improved - cf. the examples constructed in [Mss]. In the context of the examples constructed in [Mss], it is of interest to note that the estimates obtained in [Mss] for these examples appear, at first glance, to contradict the rather strong inequality obtained in the final display of Corollary 2.2 , (ii). Indeed, fix a $\xi \in \mathbb{R}$ such that $\frac{1}{2}<\xi<1$. Then if one assumes that

(1) the quantity " $\log (\mathfrak{q})$ " in the final display of Corollary 2.2, (ii), is roughly equal to the height of the elliptic curve, i.e., the relation " $\log \left(\mathfrak{q}_{(-)}\right) \approx$ ht ${ }_{\infty}$ " derived at the beginning of proof of Corollary 2.2 - which amounts, in essence, to the statement that one may ignore the contributions to the height at the archimedean primes, as well as at the primes over 2 - holds and, moreover, that

(2) the inequality in the final display of Corollary 2.2, (ii), may be applied to the elliptic curves constructed in [Mss],

then a straightforward substitution reveals that if one takes

$$
\epsilon \stackrel{\text { def }}{=}\left\{\log \left(\mathfrak{d}^{F}\right)+\log \left(\mathfrak{f}^{F}\right)\right\}^{-\xi}
$$

in the inequality in the final display of Corollary 2.2, (ii), then one obtains, at least asymptotically, a contradiction to the estimates obtained in [Mss]. In fact, it is not clear that the elliptic curves constructed in [Mss] satisfy either of the assumptions (1), (2), both of which may be thought of as assumptions to the effect that the elliptic curve in question is in "sufficiently general position". That is to say, in order to obtain elliptic curves satisfying assumptions (1), (2), one must apply the theory of [GenEll] [cf. the proofs of Corollaries 2.2, 2.3!], which involves constructing various "noncritical Belyi maps" on finite étale coverings of the projective line minus three points. Moreover, these Belyi maps and coverings depend, in an essential way, on $\epsilon$, and it is difficult to see how to bound the constants that arise in the construction of these Belyi maps and coverings in such a way as to assure that 
these constants do not affect the delicate estimates of [Mss]. In particular, despite the apparently sharper and more explicit nature [i.e., by comparison to the inequality of Corollary 2.3] of the inequality of the final display of Corollary 2.2, (ii), there is, in fact, no contradiction - as far as the author can see at the time of writing! between Corollary 2.2, (ii), and the estimates obtained in [Mss]. 


\section{Section 3: Inter-universal Formalism: the Language of Species}

In the present $\S 3$, we develop the language of species. Roughly speaking, a "species" is a "type of mathematical object", such as a "group", a "ring", a "scheme", etc. In some sense, this language may be thought of as an explicit description of certain tasks typically executed at an implicit, intuitive level by mathematicians via a sort of "mental arithmetic" in the course of interpreting various mathematical arguments. In the context of the theory developed in the present series of papers, however, it is useful to describe these intuitive operations explicitly.

In the following discussion, we shall work with various models - consisting of "sets" and a relation " $\in$ " — of the standard ZFC axioms of axiomatic set theory [i.e., the nine axioms of Zermelo-Fraenkel, together with the axiom of choice cf., e.g., [Drk], Chapter 1, §3]. We shall refer to such models as ZFC-models. Recall that a (Grothendieck) universe $V$ is a set satisfying the following axioms [cf. [McLn], p. 194]:

(i) $V$ is transitive, i.e., if $y \in x, x \in V$, then $y \in V$.

(ii) The set of natural numbers $\mathbb{N} \in V$.

(iii) If $x \in V$, then the power set of $x$ also belongs to $V$.

(iv) If $x \in V$, then the union of all members of $x$ also belongs to $V$.

(v) If $x \in V, y \subseteq V$, and $f: x \rightarrow y$ is a surjection, then $y \in V$.

We shall say that a set $E$ is a $V$-set if $E \in V$.

The various ZFC-models that we work with may be thought of as [but are not restricted to be!] the ZFC-models determined by various universes that are sets relative to some ambient ZFC-model which, in addition to the standard axioms of ZFC set theory, satisfies the following existence axiom [attributed to the "Grothendieck school" — cf. the discussion of [McLn], p. 193]:

$\left(\dagger^{\mathrm{G}}\right)$ Given any set $x$, there exists a universe $V$ such that $x \in V$.

We shall refer to a ZFC-model that also satisfies this additional axiom of the Grothendieck school as a $Z F C G$-model. This existence axiom $\left(\dagger^{\mathrm{G}}\right)$ implies, in particular, that:

Given a set $I$ and a collection of universes $V_{i}$ (where $i \in I$ ), indexed by $I$ [i.e., a 'function' $I \ni i \mapsto V_{i}$ ], there exists a [larger] universe $V$ such that $V_{i} \in V$, for $i \in I$.

Indeed, since the graph of the function $I \ni i \mapsto V_{i}$ is a set, it follows that $\left\{V_{i}\right\}_{i \in I}$ is a set. Thus, it follows from the existence axiom $\left(\dagger^{\mathrm{G}}\right)$ that there exists a universe $V$ such that $\left\{V_{i}\right\}_{i \in I} \in V$. Hence, by condition (i), we conclude that $V_{i} \in V$, for all $i \in I$, as desired. Note that this means, in particular, that there exist infinite ascending chains of universes

$$
V_{0} \in V_{1} \in V_{2} \in V_{3} \in \ldots \in V_{n} \in \ldots \in V
$$


- where $n$ ranges over the natural numbers. On the other hand, by the axiom of foundation, there do not exist infinite descending chains of universes

$$
V_{0} \ni V_{1} \ni V_{2} \ni V_{3} \ni \ldots \ni V_{n} \ni \ldots
$$

— where $n$ ranges over the natural numbers.

Although we shall not discuss in detail here the quite difficult issue of whether or not there actually exist ZFCG-models, we remark in passing that one may justify the stance of ignoring such issues - at least from the point of view of establishing the validity of various "final results" that may be formulated in ZFC-models — by invoking a result of Feferman [cf. [Ffmn], §2.3] concerning the "conservative extensionality" of ZFCG relative to ZFC, i.e., roughly speaking, that "any proposition that may be formulated in a ZFC-model and, moreover, holds in a ZFCG-model in fact holds in the original ZFC-model".

In the following discussion, we use the phrase "set-theoretic formula" as it is conventionally used in discussions of axiomatic set-theory [cf., e.g., [Drk], Chapter $1, \S 2]$.

\section{Definition 3.1.}

(i) A 0 -species $\mathfrak{S}_{0}$ is a collection of conditions given by a set-theoretic formula

$$
\Phi_{0}(\mathfrak{E})
$$

involving an ordered collection $\mathfrak{E}=\left(\mathfrak{E}_{1}, \ldots, \mathfrak{E}_{n_{0}}\right)$ of sets $\mathfrak{E}_{1}, \ldots, \mathfrak{E}_{n_{0}}$ [which we think of as "indeterminates"], for some integer $n_{0} \geq 1$; in this situation, we shall refer to $\mathfrak{E}$ as a collection of species-data for $\mathfrak{S}_{0}$. If $\mathfrak{S}_{0}$ is a 0 -species given by a set-theoretic formula $\Phi_{0}(\mathfrak{E})$, then a 0 -specimen of $\mathfrak{S}_{0}$ is a specific ordered collection of $n_{0}$ sets $E=\left(E_{1}, \ldots, E_{n_{0}}\right)$ in some specific ZFC-model that satisfies $\Phi_{0}(E)$. If $E$ is a 0 -specimen of a 0 -species $\mathfrak{S}_{0}$, then we shall write $E \in \mathfrak{S}_{0}$. If, moreover, it holds, in any ZFC-model, that the 0 -specimens of $\mathfrak{S}_{0}$ form a set, then we shall refer to $\mathfrak{S}_{0}$ as 0 -small.

(ii) Let $\mathfrak{S}_{0}$ be a 0 -species. Then a 1 -species $\mathfrak{S}_{1}$ acting on $\mathfrak{S}_{0}$ is a collection of set-theoretic formulas $\Phi_{1}, \Phi_{1 \circ 1}$ satisfying the following conditions:

(a) $\Phi_{1}$ is a set-theoretic formula

$$
\Phi_{1}\left(\mathfrak{E}, \mathfrak{E}^{\prime}, \mathfrak{F}\right)
$$

involving two collections of species-data $\mathfrak{E}, \mathfrak{E}^{\prime}$ for $\mathfrak{S}_{0}$ [i.e., the conditions $\Phi_{0}(\mathfrak{E}), \Phi_{0}\left(\mathfrak{E}^{\prime}\right)$ hold] and an ordered collection $\mathfrak{F}=\left(\mathfrak{F}_{1}, \ldots, \mathfrak{F}_{n_{1}}\right)$ of ["indeterminate"] sets $\mathfrak{F}_{1}, \ldots, \mathfrak{F}_{n_{1}}$, for some integer $n_{1} \geq 1$; in this situation, we shall refer to $\left(\mathfrak{E}, \mathfrak{E}^{\prime}, \mathfrak{F}\right)$ as a collection of species-data for $\mathfrak{S}_{1}$ and write $\mathfrak{F}: \mathfrak{E} \rightarrow \mathfrak{E}^{\prime}$. If, in some ZFC-model, $E, E^{\prime} \in \mathfrak{S}_{0}$, and $F$ is a specific ordered collection of $n_{1}$ sets that satisfies the condition $\Phi_{1}\left(E, E^{\prime}, F\right)$, then we shall refer to the data $\left(E, E^{\prime}, F\right)$ as a 1 -specimen of $\mathfrak{S}_{1}$ and write $\left(E, E^{\prime}, F\right) \in \mathfrak{S}_{1}$; alternatively, we shall denote a 1 -specimen $\left(E, E^{\prime}, F\right)$ via 
the notation $F: E \rightarrow E^{\prime}$ and refer to $E$ (respectively, $E^{\prime}$ ) as the domain (respectively, codomain) of $F: E \rightarrow E^{\prime}$.

(b) $\Phi_{1 \circ 1}$ is a set-theoretic formula

$$
\Phi_{1 \circ 1}\left(\mathfrak{E}, \mathfrak{E}^{\prime}, \mathfrak{E}^{\prime \prime}, \mathfrak{F}, \mathfrak{F}^{\prime}, \mathfrak{F}^{\prime \prime}\right)
$$

involving three collections of species-data $\mathfrak{F}: \mathfrak{E} \rightarrow \mathfrak{E}^{\prime}, \mathfrak{F}^{\prime}: \mathfrak{E}^{\prime} \rightarrow \mathfrak{E}^{\prime \prime}, \mathfrak{F}^{\prime \prime}:$ $\mathfrak{E} \rightarrow \mathfrak{E}^{\prime \prime}$ for $\mathfrak{S}_{1}$ [i.e., the conditions $\Phi_{0}(\mathfrak{E}) ; \Phi_{0}\left(\mathfrak{E}^{\prime}\right) ; \Phi_{0}\left(\mathfrak{E}^{\prime \prime}\right) ; \Phi_{1}\left(\mathfrak{E}, \mathfrak{E}^{\prime}, \mathfrak{F}\right) ;$ $\Phi_{1}\left(\mathfrak{E}^{\prime}, \mathfrak{E}^{\prime \prime}, \mathfrak{F}^{\prime}\right) ; \Phi_{1}\left(\mathfrak{E}, \mathfrak{E}^{\prime \prime}, \mathfrak{F}^{\prime \prime}\right)$ hold]; in this situation, we shall refer to $\mathfrak{F}^{\prime \prime}$ as a composite of $\mathfrak{F}$ with $\mathfrak{F}^{\prime}$ and write $\mathfrak{F}^{\prime \prime}=\mathfrak{F}^{\prime} \circ \mathfrak{F}$ [which is, a priori, an abuse of notation, since there may exist many composites of $\mathfrak{F}$ with $\mathfrak{F}^{\prime}-$ cf. (c) below]; we shall use similar terminology and notation for 1-specimens in specific ZFC-models.

(c) Given a pair of 1-specimens $F: E \rightarrow E^{\prime}, F^{\prime}: E^{\prime} \rightarrow E^{\prime \prime}$ of $\mathfrak{S}_{1}$ in some ZFC-model, there exists a unique composite $F^{\prime \prime}: E \rightarrow E^{\prime \prime}$ of $F$ with $F^{\prime}$ in the given ZFC-model.

(d) Composition of 1-specimens $F: E \rightarrow E^{\prime}, F^{\prime}: E^{\prime} \rightarrow E^{\prime \prime}, F^{\prime \prime}: E^{\prime \prime} \rightarrow E^{\prime \prime \prime}$ of $\mathfrak{S}_{1}$ in a ZFC-model is associative.

(e) For any 0 -specimen $E$ of $\mathfrak{S}_{0}$ in a ZFC-model, there exists a [necessarily unique] 1-specimen $F: E \rightarrow E$ of $\mathfrak{S}_{1}$ [in the given ZFC-model] — which we shall refer to as the identity 1-specimen $\operatorname{id}_{E}$ of $E$ - such that for any 1-specimens $F^{\prime}: E^{\prime} \rightarrow E, F^{\prime \prime}: E \rightarrow E^{\prime \prime}$ of $\mathfrak{S}_{1}$ [in the given ZFC-model] we have $F \circ F^{\prime}=F^{\prime}, F^{\prime \prime} \circ F=F^{\prime \prime}$.

If, moreover, it holds, in any ZFC-model, that for any two 0-specimens $E, E^{\prime}$ of $\mathfrak{S}_{0}$, the 1-specimens $F: E \rightarrow E^{\prime}$ of $\mathfrak{S}_{1}$ [i.e., the 1-specimens of $\mathfrak{S}_{1}$ with domain $E$ and codomain $\left.E^{\prime}\right]$ form a set, then we shall refer to $\mathfrak{S}_{1}$ as 1-small.

(iii) A species $\mathfrak{S}$ is defined to be a pair consisting of a 0 -species $\mathfrak{S}_{0}$ and a $1-$ species $\mathfrak{S}_{1}$ acting on $\mathfrak{S}_{0}$. Fix a species $\mathfrak{S}=\left(\mathfrak{S}_{0}, \mathfrak{S}_{1}\right)$. Let $i \in\{0,1\}$. Then we shall refer to an $i$-specimen of $\mathfrak{S}_{i}$ as an $i$-specimen of $\mathfrak{S}$. We shall refer to a 0 -specimen (respectively, 1-specimen) of $\mathfrak{S}$ as a species-object (respectively, a species-morphism) of $\mathfrak{S}$. We shall say that $\mathfrak{S}$ is $i$-small if $\mathfrak{S}_{i}$ is $i$-small. We shall refer to a speciesmorphism $F: E \rightarrow E^{\prime}$ as a species-isomorphism if there exists a species-morphism $F^{\prime}: E^{\prime} \rightarrow E$ such that the composites $F \circ F^{\prime}, F^{\prime} \circ F$ are identity species-morphisms; in this situation, we shall say that $E, E^{\prime}$ are species-isomorphic. [Thus, one verifies immediately that composites of species-isomorphisms are species-isomorphisms.] We shall refer to a species-isomorphism whose domain and codomain are equal as a species-automorphism. We shall refer to as model-free [cf. Remark 3.1.1 below] an $i$-specimen of $\mathfrak{S}$ equipped with a description via a set-theoretic formula that is "independent of the ZFC-model in which it is given" in the sense that for any pair of universes $V_{1}, V_{2}$ of some ZFC-model such that $V_{1} \in V_{2}$, the set-theoretic formula determines the same $i$-specimen of $\mathfrak{S}$, whether interpreted relative to the ZFC-model determined by $V_{1}$ or the ZFC-model determined by $V_{2}$.

(iv) We shall refer to as the category determined by $\mathfrak{S}$ in a ZFC-model the category whose objects are the species-objects of $\mathfrak{S}$ in the given ZFC-model and 
whose arrows are the species-morphisms of $\mathfrak{S}$ in the given ZFC-model. [One verifies immediately that this description does indeed determine a category.]

Remark 3.1.1. We observe that any of the familiar descriptions of $\mathbb{N}$ [cf., e.g., [Drk], Chapter 2, Definition 2.3], $\mathbb{Z}, \mathbb{Q}, \mathbb{Q}_{p}$, or $\mathbb{R}$, for instance, yield species [all of whose species-morphisms are identity species-morphisms] each of which has a unique species-object in any given ZFC-model. Such species are not to be confused with such species as the species of "monoids isomorphic to $\mathbb{N}$ and monoid isomorphisms", which admits many species-objects [all of which are species-isomorphic] in any ZFCmodel. On the other hand, the set-theoretic formula used, for instance, to define the former "species $\mathbb{N}$ " may be applied to define a "model-free species-object $\mathbb{N}$ " of the latter "species of monoids isomorphic to $\mathbb{N}$ ".

\section{Remark 3.1.2.}

(i) It is important to remember when working with species that

the essence of a species lies not in the specific sets that occur as speciesobjects or species-morphisms of the species in various ZFC-models, but rather in the collection of rules, i.e., set-theoretic formulas, that govern the construction of such sets in an unspecified, "indeterminate" ZFCmodel.

Put another way, the emphasis in the theory of species lies in the programs - i.e., "software" - that yield the desired output data, not on the output data itself. From this point of view, one way to describe the various set-theoretic formulas that constitute a species is as a "deterministic algorithm" [a term suggested to the author by M. Kim] for constructing the sets to be considered.

(ii) One interesting point of view that arose in discussions between the author and F. Kato is the following. The relationship between the classical "set-theoretic" approach to discussing mathematics — in which specific sets play a central role and the "species-theoretic" approach considered here - in which the rules, given by set-theoretic formulas for constructing the sets of interest [i.e., not specific sets themselves!], play a central role - may be regarded as analogous to the relationship between classical approaches to algebraic varieties - in which specific sets of solutions of polynomial equations in an algebraically closed field play a central role - and scheme theory - in which the functor determined by a scheme, i.e., the polynomial equations, or "rules", that determine solutions, as opposed to specific sets of solutions themselves, play a central role. That is to say, in summary:

[set-th. approach : species-th. approach] $\longleftrightarrow$ [varieties : schemes]

A similar analogy — i.e., of the form

[set-th. approach : species-th. approach]

$\longleftrightarrow \quad$ [groups of specific matrices : abstract groups] 
- may be made to the notion of an "abstract group", as opposed to a "group of specific matices". That is to say, just as a "group of specific matrices may be thought of as a specific representation of an "abstract group", the category of objects determined by a species in a specific ZFC-model may be thought of as a specific representation of an "abstract species".

(iii) If, in the context of the discussion of (i), (ii), one tries to form a sort of quotient, in which "programs" that yield the same sets as "output data" are identified, then one must contend with the resulting indeterminacy, i.e., working with programs is only well-defined up to internal modifications of the programs in question that does not affect the final output. This leads to somewhat intractable problems concerning the internal structure of such programs - a topic that lies well beyond the scope of the present work.

\section{Remark 3.1.3.}

(i) Typically, in the discussion to follow, we shall not write out explicitly the various set-theoretic formulas involved in the definition of a species. Rather, it is to be understood that the set-theoretic formulas to be used are those arising from the conventional descriptions of the mathematical objects involved. When applying such conventional descriptions, however, it is important to check that they are well-defined and do not depend upon the use of arbitrary choices that are not describable via well-defined set-theoretic formulas.

(ii) The fact that the data involved in a species is given by abstract set-theoretic formulas imparts a certain canonicality to the mathematical notion constituted by the species, a canonicality that is not shared, for instance, by mathematical objects whose construction depends on an invocation of the axiom of choice in some particular ZFC-model [cf. the discussion of (i) above]. Moreover, by furnishing a stock of such "canonical notions", the theory of species allows one, in effect, to compute the extent of deviation of various "non-canonical objects" [i.e., whose construction depends upon the invocation of the axiom of choice!] from a sort of "canonical norm".

Remark 3.1.4. Note that because the data involved in a species is given by abstract set-theoretic formulas, the mathematical notion constituted by the species is immune to, i.e., unaffected by, extensions of the universe - i.e., such as the ascending chain $V_{0} \in V_{1} \in V_{2} \in V_{3} \in \ldots \in V_{n} \in \ldots \in V$ that appears in the discussion preceding Definition 3.1 - in which one works. This is the sense in which we apply the term "inter-universal". That is to say, "inter-universal geometry" allows one to relate the "geometries" that occur in distinct universes.

Remark 3.1.5. Similar remarks to the remarks made in Remarks 3.1.2, 3.1.3, and 3.1.4 concerning the significance of working with set-theoretic formulas may be made with regard to the notions of mutations, morphisms of mutations, mutationhistories, observables, and cores to be introduced in Definition 3.2 below.

One fundamental example of a species is the following. 
Example 3.2. Categories. The notions of a [small] category and an isomorphism class of [covariant] functors between two given [small] categories yield an example of a species. That is to say, at a set-theoretic level, one may think of a [small] category as, for instance, a set of arrows, together with a set of composition relations, that satisfies certain properties; one may think of a [covariant] functor between [small] categories as the set given by the graph of the map on arrows determined by the functor [which satisfies certain properties]; one may think of an isomorphism class of functors as a collection of such graphs, i.e., the graphs determined by the functors in the isomorphism class, which satisfies certain properties. Then one has "dictionaries"

0-species $\longleftrightarrow \quad$ the notion of a category

1-species $\longleftrightarrow \quad$ the notion of an isomorphism class of functors

at the level of notions and

$$
\text { a } 0 \text {-specimen } \longleftrightarrow \text { a particular [small] category }
$$

a 1-specimen $\longleftrightarrow$ a particular isomorphism class of functors

at the level of specific mathematical objects in a specific ZFC-model. Moreover, one verifies easily that species-isomorphisms between 0 -species correspond to isomorphism classes of equivalences of categories in the usual sense.

Remark 3.2.1. Note that in the case of Example 3.2, one could also define a notion of "2-species", "2-specimens", etc., via the notion of an "isomorphism of functors", and then take the 1-species under consideration to be the notion of a functor [i.e., not an isomorphism class of functors]. Indeed, more generally, one could define a notion of " $n$-species" for arbitrary integers $n \geq 1$. Since, however, this approach would only serve to add an unnecessary level of complexity to the theory, we choose here to take the approach of working with "functors considered up to isomorphism".

Definition 3.3. Let $\mathfrak{S}=\left(\mathfrak{S}_{0}, \mathfrak{S}_{1}\right) ; \underline{\mathfrak{S}}=\left(\underline{\mathfrak{S}}_{0}, \underline{\mathfrak{S}}_{1}\right) ; \underline{\underline{\mathfrak{S}}}=\left(\underline{\underline{\mathfrak{S}}}_{0}, \underline{\underline{\mathfrak{S}}}_{1}\right)$ be species.

(i) A mutation $\mathfrak{M}: \mathfrak{S} \rightsquigarrow \underline{\mathfrak{S}}$ is defined to be a collection of set-theoretic formulas $\Psi_{0}, \Psi_{1}$ satisfying the following properties:

(a) $\Psi_{0}$ is a set-theoretic formula

$$
\Psi_{0}(\mathfrak{E}, \underline{\mathfrak{E}})
$$

involving a collection of species-data $\mathfrak{E}$ for $\mathfrak{S}_{0}$ and a collection of speciesdata $\underline{\mathfrak{E}}$ for $\underline{\mathfrak{S}}_{0}$; in this situation, we shall write $\mathfrak{M}(\mathfrak{E})$ for $\underline{\mathfrak{E}}$. Moreover, if, in some ZFC-model, $E \in \mathfrak{S}_{0}$, then we require that there exist a unique $\underline{E} \in \underline{\mathfrak{S}}_{0}$ such that $\Psi_{0}(E, \underline{E})$ holds; in this situation, we shall write $\mathfrak{M}(E)$ for $\underline{E}$.

(b) $\Psi_{1}$ is a set-theoretic formula

$$
\Psi_{1}\left(\mathfrak{E}, \mathfrak{E}^{\prime}, \mathfrak{F}, \underline{\mathfrak{F}}\right)
$$


involving a collection of species-data $\mathfrak{F}: \mathfrak{E} \rightarrow \mathfrak{E}^{\prime}$ for $\mathfrak{S}_{1}$ and a collection of species-data $\underline{\mathfrak{F}}: \underline{\mathfrak{E}} \rightarrow \underline{\mathfrak{E}}^{\prime}$ for $\underline{\mathfrak{S}}_{1}$, where $\underline{\mathfrak{E}}=\mathfrak{M}(\mathfrak{E}), \underline{\mathfrak{E}}^{\prime}=\mathfrak{M}\left(\mathfrak{E}^{\prime}\right)$; in this situation, we shall write $\mathfrak{M}(\mathfrak{F})$ for $\mathfrak{F}$. Moreover, if, in some ZFCmodel, $\left(F: E \rightarrow E^{\prime}\right) \in \mathfrak{S}_{1}$, then we require that there exist a unique $\left(\underline{F}: \underline{E} \rightarrow \underline{E}^{\prime}\right) \in \underline{\mathfrak{S}}_{1}$ such that $\Psi_{0}\left(E, E^{\prime}, F, \underline{F}\right)$ holds; in this situation, we shall write $\mathfrak{M}(F)$ for $\underline{F}$. Finally, we require that the assignment $F \mapsto$ $\mathfrak{M}(F)$ be compatible with composites and map identity species-morphisms of $\mathfrak{S}$ to identity species-morphisms of $\underline{\mathfrak{S}}$. In particular, if one fixes a ZFCmodel, then $\mathfrak{M}$ determines a functor from the category determined by $\mathfrak{S}$ in the given ZFC-model to the category determined by $\underline{\mathfrak{S}}$ in the given ZFC-model.

There are evident notions of "composition of mutations" and "identity mutations".

(ii) Let $\mathfrak{M}, \mathfrak{M}^{\prime}: \mathfrak{S} \rightsquigarrow \mathfrak{S}$ be mutations. Then a morphism of mutations $\mathfrak{Z}: \mathfrak{M} \rightarrow \mathfrak{M}^{\prime}$ is defined to be a set-theoretic formula $\Xi$ satisfying the following properties:

(a) $\Xi$ is a set-theoretic formula

$$
\Xi(\mathfrak{E}, \underline{\mathfrak{F}})
$$

involving a collection of species-data $\mathfrak{E}$ for $\mathfrak{S}_{0}$ and a collection of speciesdata $\underline{\mathfrak{F}}: \mathfrak{M}(\mathfrak{E}) \rightarrow \mathfrak{M}^{\prime}(\mathfrak{E})$ for $\mathfrak{S}_{1}$; in this situation, we shall write $\mathfrak{Z}(\mathfrak{E})$ for $\underline{\mathfrak{F}}$. Moreover, if, in some ZFC-model, $E \in \mathfrak{S}_{0}$, then we require that there exist a unique $\underline{F} \in \underline{\mathfrak{S}}_{1}$ such that $\Xi(E, \underline{F})$ holds; in this situation, we shall write $\mathfrak{Z}(E)$ for $\underline{F}$.

(b) Suppose, in some ZFC-model, that $F: E_{1} \rightarrow E_{2}$ is a species-morphism of $\mathfrak{S}$. Then one has an equality of composite species-morphisms $\mathfrak{M}^{\prime}(F) \circ$ $\mathfrak{Z}\left(E_{1}\right)=\mathfrak{Z}\left(E_{2}\right) \circ \mathfrak{M}(F): \mathfrak{M}\left(E_{1}\right) \rightarrow \mathfrak{M}^{\prime}\left(E_{2}\right)$. In particular, if one fixes a ZFC-model, then a morphism of mutations $\mathfrak{M} \rightarrow \mathfrak{M}^{\prime}$ determines a natural transformation between the functors determined by $\mathfrak{M}, \mathfrak{M}^{\prime}$ in the ZFCmodel - cf. (i).

There are evident notions of "composition of morphisms of mutations" and "identity morphisms of mutations". If it holds that for every species-object $E$ of $\mathfrak{S}, \mathfrak{Z}(E)$ is a species-isomorphism, then we shall refer to $\mathfrak{Z}$ as an isomorphism of mutations. In particular, one verifies immediately that $\mathfrak{Z}$ is an isomorphism of mutations if and only if there exists a morphism of mutations $\mathfrak{Z}^{\prime}: \mathfrak{M}^{\prime} \rightarrow \mathfrak{M}$ such that the composite morphisms of mutations $\mathfrak{Z}^{\prime} \circ \mathfrak{Z}: \mathfrak{M} \rightarrow \mathfrak{M}, \mathfrak{Z} \circ \mathfrak{Z}^{\prime}: \mathfrak{M}^{\prime} \rightarrow \mathfrak{M}^{\prime}$ are the respective identity morphisms of the mutations $\mathfrak{M}, \mathfrak{M}^{\prime}$.

(iii) Let $\mathfrak{M}: \mathfrak{S} \rightsquigarrow \underline{\mathfrak{S}}$ be a mutation. Then we shall say that $\mathfrak{M}$ is a mutationequivalence if there exists a mutation $\mathfrak{M}^{\prime}: \underline{\mathfrak{S}} \rightsquigarrow \mathfrak{S}$, together with isomorphisms of mutations between the composites $\mathfrak{M} \circ \mathfrak{M}^{\prime}, \mathfrak{M}^{\prime} \circ \mathfrak{M}$ and the respective identity mutations. In this situation, we shall say that $\mathfrak{M}, \mathfrak{M}^{\prime}$ are mutation-quasi-inverses to one another. Note that for any two given species-objects in the domain species of a mutation-equivalence, the mutation-equivalence induces a bijection between 
the collection of species-isomorphisms between the two given species-objects [of the domain species] and the collection of species-isomorphisms between the two speciesobjects [of the codomain species] obtained by applying the mutation-equivalence to the two given species-objects.

(iv) Let $\vec{\Gamma}$ be an oriented graph, i.e., a graph $\Gamma$, which we shall refer to as the underlying graph of $\vec{\Gamma}$, equipped with the additional data of a total ordering, for each edge $e$ of $\Gamma$, on the set [of cardinality 2] of branches of $e$ [cf., e.g., [AbsTopIII], $\S 0]$. Then we define a mutation-history $\mathfrak{H}=\left(\vec{\Gamma}, \mathfrak{S}^{*}, \mathfrak{M}^{*}\right)$ [indexed by $\left.\vec{\Gamma}\right]$ to be a collection of data as follows:

(a) for each vertex $v$ of $\vec{\Gamma}$, a species $\mathfrak{S}^{v}$;

(b) for each edge $e$ of $\vec{\Gamma}$, running from a vertex $v_{1}$ to a vertex $v_{2}$, a mutation $\mathfrak{M}^{e}: \mathfrak{S}^{v_{1}} \rightsquigarrow \mathfrak{S}^{v_{2}}$.

In this situation, we shall refer to the vertices, edges, and branches of $\vec{\Gamma}$ as vertices, edges, and branches of $\mathfrak{H}$. Thus, the notion of a "mutation-history" may be thought of as a species-theoretic version of the notion of a "diagram of categories" given in [AbsTopIII], Definition 3.5, (i).

(v) Let $\mathfrak{H}=\left(\vec{\Gamma}, \mathfrak{S}^{*}, \mathfrak{M}^{*}\right)$ be a mutation-history; $\underline{\mathfrak{S}}$ a species. For simplicity, we assume that the underlying graph of $\vec{\Gamma}$ is simply connected. Then we shall refer to as a(n) [్-valued] covariant (respectively, contravariant) observable $\mathfrak{V}$ of the mutation-history $\mathfrak{H}$ a collection of data as follows:

(a) for each vertex $v$ of $\vec{\Gamma}$, a mutation $\mathfrak{V}^{v}: \mathfrak{S}^{v} \rightarrow \underline{\mathfrak{S}}$, which we shall refer to as the observation mutation at $v$;

(b) for each edge $e$ of $\vec{\Gamma}$, running from a vertex $v_{1}$ to a vertex $v_{2}$, a morphism of mutations $\mathfrak{V}^{e}: \mathfrak{V}^{v_{1}} \rightarrow \mathfrak{V}^{v_{2}} \circ \mathfrak{M}^{e}$ (respectively, $\mathfrak{V}^{e}: \mathfrak{V}^{v_{2}} \circ \mathfrak{M}^{e} \rightarrow \mathfrak{V}^{v_{1}}$ ).

If $\mathfrak{V}$ is a covariant observable such that all of the morphisms of mutations " $\mathfrak{V}$ " are isomorphisms of mutations, then we shall refer to the covariant observable $\mathfrak{V}$ as a core. Thus, one may think of a core $\mathfrak{C}$ of a mutation-history as lying "under" the entire mutation-history in a "uniform fashion". Also, we shall refer to the "property [of an observable] of being a core" as the "coricity" of the observable. Finally, we note that the notions of an "observable" and a "core" given here may be thought of as simplified, species-theoretic versions of the notions of "observable" and "core" given in [AbsTopIII], Definition 3.5, (iii).

\section{Remark 3.3.1.}

(i) One well-known consequence of the axiom of foundation of axiomatic settheory is the assertion that " $\in$-loops"

$$
a \in b \in c \in \ldots \in a
$$


can never occur in the set theory in which one works. On the other hand, there are many situations in mathematics in which one wishes to somehow "identify" mathematical objects that arise at higher levels of the $\in$-structure of the set theory under consideration with mathematical objects that arise at lower levels of this $\in$-structure. In some sense, the notions of a "set" and of a "bijection of sets" allow one to achieve such "identifications". That is to say, the mathematical objects at both higher and lower levels of the $\in$-structure constitute examples of the same mathematical notion of a "set", so that one may consider "bijections of sets" between those sets without violating the axiom of foundation. In some sense, the notion of a species may be thought of as a natural extension of this observation. That is to say,

the notion of a "species" allows one to consider, for instance, speciesisomorphisms between species-objects that occur at different levels of the $\in$-structure of the set theory under consideration - i.e., roughly speaking, to "simulate $\in$-loops" - without violating the axiom of foundation.

Moreover, typically the species-objects at higher levels of the $\in$-structure occur as the result of executing the mutations that arise in some sort of mutation-history

$$
\ldots \rightsquigarrow \mathfrak{S} \rightsquigarrow \underline{\mathfrak{S}} \rightsquigarrow \underline{\underline{S}} \rightsquigarrow \ldots \rightsquigarrow \mathfrak{S} \rightsquigarrow \ldots
$$

— e.g., the "output species-objects" of the "్" on the right that arise from applying various mutations to the "input species-objects" of the "్" on the left.

(ii) In the context of constructing "loops" in a mutation-history as in the final display of (i), we observe that

the simpler the structure of the species involved, the easier it is to construct "loops".

It is for this reason that species such as the species determined by the notion of a category [cf. Example 3.2] are easier to work with, from the point of view of constructing "loops", than more complicated species such as the species determined by the notion of a scheme. This is one of the principal motivations for the "geometry of categories" - of which "absolute anabelian geometry" is the special case that arises when the categories involved are Galois categories - i.e., for the theory of representing scheme-theoretic geometries via categories [cf., e.g., the Introductions of [LgSch], [ArLgSch], [SemiAnbd], [Cusp], [FrdI]]. At a more concrete level, the utility of working with categories to reconstruct objects that occurred at lower levels of some sort of "series of constructions" [cf. the mutation-history of the final display of (i)!] may be seen in the "reconstruction of the underlying scheme", given in [LgSch], Corollary 2.15, from a certain category constructed from a log scheme, as well as in the theory of "slim exponentiation" discussed in the Appendix to [FrdI].

(iii) Again in the context of mutation-histories such as the one given in the final display of (i), although one may, on certain occasions, wish to apply various mutations that fundamentally alter the structure of the mathematical objects involved and hence give rise to "output species-objects" of the "్" on the right that 
are related in a highly nontrivial fashion to the "input species-objects" of the "్" on the left, it is also of interest to consider

"portions" of the various mathematical objects that occur that are left unaltered by the various mutations that one applies.

This is precisely the reason for the introduction of the notion of a core of a mutationhistory. One important consequence of the construction of various cores associated to a mutation-history is that often

one may apply various cores associated to a mutation-history to describe, by means of non-coric observables, the portions of the various mathematical objects that occur which are altered by the various mutations that one applies in terms of the unaltered portions, i.e., cores.

Indeed, this point of view plays a central role in the theory of the present series of papers - cf. the discussion of Remark 3.6.1, (ii), below.

Remark 3.3.2. $\quad$ One somewhat naive point of view that constituted one of the original motivations for the author in the development of theory of the present series of papers is the following. In the classical theory of schemes, when considering local systems on a scheme, there is no reason to restrict oneself to considering local systems valued in, say, modules over a finite ring. If, moreover, there is no reason to make such a restriction, then one is naturally led to consider, for instance, local systems of schemes [cf., e.g., the theory of the "Galois mantle" in [ $p$ Teich]], or, indeed, local systems of "entire set-theoretic mathematical theaters". One may then ask what happens if one tries to consider local systems on the schemes that occur as fibers of a local system of schemes. [More concretely, if $X$ is, for instance, a connected scheme, then one may consider local systems $\mathcal{X}$ over $X$ whose fibers are isomorphic to $X$; then one may repeat this process, by considering such local systems over each fiber of the local system $\mathcal{X}$ on $X$, etc.] In this way, one is eventually led to the consideration of "systems of nested local systems" i.e., a local system over a local system over a local system, etc. It is precisely this point of view that underlies the notion of "successive iteration of a given mutationhistory", relative to the terminology formulated in the present $\S 3$. If, moreover, one thinks of such "successive iterates of a given mutation-history" as being a sort of abstraction of the naive idea of a "system of nested local systems", then the notion of a core may be thought of as a sort of mathematical object that is invariant with respect to the application of the operations that gave rise to the "system of nested local systems".

\section{Example 3.4. Topological Spaces and Fundamental Groups.}

(i) One verifies easily that the notions of a topological space and a continuous map between topological spaces determine an example of a species $\mathfrak{S}^{\text {top }}$. In a similar vein, the notions of a universal covering $\widetilde{X} \rightarrow X$ of a pathwise connected topological space $X$ and a continuous map between such universal coverings $\widetilde{X} \rightarrow X, \widetilde{Y} \rightarrow Y$ [i.e., a pair of compatible continuous maps $\widetilde{X} \rightarrow \widetilde{Y}, X \rightarrow Y$ ], considered up to 
composition with a deck transformation of the universal covering $\widetilde{Y} \rightarrow Y$, determine an example of a species $\mathfrak{S}^{\mathrm{u}-\mathrm{top}}$. We leave to the reader the routine task of writing out the various set-theoretic formulas that define the species structures of $\mathfrak{S}^{\text {top }}$, $\mathfrak{S}^{\text {u-top }}$. Here, we note that at a set-theoretic level, the species-morphisms of $\mathfrak{S}^{\text {u-top }}$ are collections of continuous maps [between two given universal coverings], any two of which differ from one another by composition with a deck transformation.

(ii) One verifies easily that the notions of a group and an outer homomorphism between groups [i.e., a homomorphism considered up to composition with an inner automorphism of the codomain group] determine an example of a species $\mathfrak{S}^{\mathrm{gp}}$. We leave to the reader the routine task of writing out the various set-theoretic formulas that define the species structure of $\mathfrak{S}^{\mathrm{gp}}$. Here, we note that at a set-theoretic level, the species-morphisms of $\mathfrak{S}^{\mathrm{gp}}$ are collections of homomorphisms [between two given groups], any two of which differ from one another by composition with an inner automorphism.

(iii) Now one verifies easily that the assignment

$$
(\tilde{X} \rightarrow X) \mapsto \operatorname{Aut}(\tilde{X} / X)
$$

— where $(\tilde{X} \rightarrow X)$ is a species-object of $\mathfrak{S}^{\text {u-top }}$, and $\operatorname{Aut}(\tilde{X} / X)$ denotes the group of deck transformations of the universal covering $\widetilde{X} \rightarrow X$ - determines a mutation $\mathfrak{S}^{\mathrm{u}-\mathrm{top}} \rightsquigarrow \mathfrak{S}^{\mathrm{gp}}$. That is to say, the "fundamental group" may be thought of as a sort of mutation.

\section{Example 3.5. Absolute Anabelian Geometry.}

(i) Let $\mathcal{S}$ be a class of connected normal schemes that is closed under isomorphism [of schemes]. Suppose that there exists a set $E_{\mathcal{S}}$ of schemes describable by a set-theoretic formula with the property that every scheme of $\mathcal{S}$ is isomorphic to some scheme belonging to $E_{\mathcal{S}}$. Then just as in the case of universal coverings of topological spaces discussed in Example 3.4, (i), one verifies easily, by applying the set-theoretic formula describing $E_{\mathcal{S}}$, that the universal pro-finite étale coverings $\widetilde{X} \rightarrow X$ of schemes $X$ belonging to $\mathcal{S}$ and isomorphisms of such coverings considered up to composition with a deck transformation give rise to a species $\mathfrak{S}^{\mathcal{S}}$.

(ii) Let $\mathcal{G}$ be a class of topological groups that is closed under isomorphism [of topological groups]. Suppose that there exists a set $E_{\mathcal{G}}$ of topological groups describable by a set-theoretic formula with the property that every topological group of $\mathcal{G}$ is isomorphic to some topological group belonging to $E_{\mathcal{G}}$. Then just as in the case of abstract groups discussed in Example 3.4, (ii), one verifies easily, by applying the set-theoretic formula describing $E_{\mathcal{G}}$, that topological groups belonging to $\mathcal{G}$ and [bi-continuous] outer isomorphisms between such topological groups give rise to a species $\mathfrak{S}^{\mathcal{G}}$.

(iii) Let $\mathcal{S}$ be as in (i). Then for an appropriate choice of $\mathcal{G}$, by associating to a universal pro-finite étale covering the resulting group of deck transformations, one obtains a mutation

$$
\Pi: \mathfrak{S}^{\mathcal{S}} \rightsquigarrow \mathfrak{S}^{\mathcal{G}}
$$


[cf. Example 3.4, (iii)]. Then one way to define the notion that the schemes belonging to the class $\mathcal{S}$ are "[absolute] anabelian" is to require the specification of a mutation

$$
\mathbb{A}: \mathfrak{S}^{\mathcal{G}} \rightsquigarrow \mathfrak{S}^{\mathcal{S}}
$$

which forms a mutation-quasi-inverse to $\Pi$. Here, we note that the existence of the bijections [i.e., "fully faithfulness"] discussed in Definition 3.3, (iii), is, in essence, the condition that is usually taken as the definition of "anabelian". By contrast, the species-theoretic approach of the present discussion may be thought of as an explicit mathematical formulation of the algorithmic approach to [absolute] anabelian geometry discussed in the Introduction to [AbsTopI].

(iv) The framework of [absolute] anabelian geometry [cf., e.g., the framework discussed above] gives a good example of the importance of specifying precisely what species one is working with in a given "series of constructions" [cf., e.g., the mutation-history of the final display of Remark 3.3.1, (i)]. That is to say, there is a quite substantial difference between working with a

profinite group in its sole capacity as a profinite group

and working with the same profinite group — which may happen to arise as the étale fundamental group of some scheme! -

regarded as being equipped with various data that arise from the construction of the profinite group as the étale fundamental group of some scheme.

It is precisely this sort of issue that constituted one of the original motivations for the author in the development of the theory of species presented here.

\section{Example 3.6. The Étale Site and Frobenius.}

(i) Let $p$ be a prime number. If $S$ is a reduced scheme over $\mathbb{F}_{p}$, then denote by $S^{(p)}$ the scheme with the same topological space as $S$, but whose structure sheaf is given by the subsheaf

$$
\mathcal{O}_{S(p)} \stackrel{\text { def }}{=}\left(\mathcal{O}_{S}\right)^{p} \subseteq \mathcal{O}_{S}
$$

of $p$-th powers of sections of $S$. Thus, the natural inclusion $\mathcal{O}_{S^{(p)}} \hookrightarrow \mathcal{O}_{S}$ induces a morphism $\Phi_{S}: S \rightarrow S^{(p)}$. Moreover, "raising to the $p$-th power" determines a natural isomorphism $\alpha_{S}: S^{(p)} \stackrel{\sim}{\rightarrow} S$ such that the resulting composite $\alpha_{S} \circ \Phi_{S}$ : $S \rightarrow S$ is the Frobenius morphism of $S$. Write

$$
\mathfrak{S}^{p-\mathrm{sch}}
$$

for the species of reduced schemes over $\mathbb{F}_{p}$ and morphisms of schemes. Note that by considering, for instance, [necessarily quasi-affine!] étale morphisms of finite presentation $T \rightarrow S$ equipped with factorizations $\left.T\right|_{U} \subseteq \mathbb{A}_{U}^{N} \rightarrow U$ for each affine open $U \subseteq S$ [where $\mathbb{A}_{U}^{N}$ denotes a "standard copy of affine $N$-space over $U$ ", for some integer $N \geq 1$; the " $\subseteq$ " exhibits $\left.T\right|_{U}$ as a finitely presented subscheme of $\left.\mathbb{A}_{U}^{N}\right]$, one may construct an assignment

$$
S \mapsto S_{\text {ét }}
$$


that maps a species-object $S$ of $\mathfrak{S}^{p \text {-sch }}$ to the category $S_{\text {ét }}$ of such étale morphisms of finite presentation $T \rightarrow S$ and $S$-morphisms - i.e., "the small étale site of $S$ " - in such a way that the assignment $S \mapsto S_{\text {ét }}$ is contravariantly functorial with respect to species-morphisms $S_{1} \rightarrow S_{2}$ of $\mathfrak{S}^{p \text {-sch }}$, and, moreover, may be described via set-theoretic formulas. Thus, such an assignment determines an "étale site mutation"

$$
\mathfrak{M}^{e^{e t}}: \mathfrak{S}^{p \text {-sch }} \rightsquigarrow \mathfrak{S}^{\text {cat }}
$$

- where we write $\mathfrak{S}^{\text {cat }}$ for the species of categories and isomorphism classes of contravariant functors [cf. Example 3.2]. Another natural assignment in the present context is the assignment

$$
S \mapsto S^{\mathrm{pf}}
$$

which maps $S$ to its perfection $S^{\text {pf }}$, i.e., the scheme determined by taking the inverse limit of the inverse system $\ldots \rightarrow S \rightarrow S \rightarrow S$ obtained by considering iterates of the Frobenius morphism of $S$. Thus, by considering the final copy of " $S$ " in this inverse system, one obtains a natural morphism $\beta_{S}: S^{\mathrm{pf}} \rightarrow S$. Finally, one obtains a "perfection mutation"

$$
\mathfrak{M}^{\text {pf }}: \mathfrak{S}^{p-\text { sch }} \rightsquigarrow \mathfrak{S}^{p-\text { sch }}
$$

by considering the set-theoretic formulas underlying the assignment $S \mapsto S^{\mathrm{pf}}$.

(ii) Write

$$
\mathfrak{F}^{p \text {-sch }}: \mathfrak{S}^{p \text {-sch }} \rightsquigarrow \mathfrak{S}^{p \text {-sch }}
$$

for the "Frobenius mutation" obtained by considering the set-theoretic formulas underlying the assignment $S \mapsto S^{(p)}$. Thus, one may formulate the well-known "invariance of the étale site under Frobenius" [cf., e.g., [FK], Chapter I, Proposition 3.16] as the statement that the "étale site mutation" $\mathfrak{M}^{\text {ét }}$ exhibits $\mathfrak{S}^{\text {cat }}$ as a core — i.e., an "invariant piece" — of the "Frobenius mutation-history"

$$
\ldots \rightsquigarrow \mathfrak{S}^{p \text {-sch }} \rightsquigarrow \mathfrak{S}^{p \text {-sch }} \rightsquigarrow \mathfrak{S}^{p \text {-sch }} \rightsquigarrow \mathfrak{S}^{p \text {-sch }} \rightsquigarrow \ldots
$$

determined by the "Frobenius mutation" $\mathfrak{F}^{p \text {-sch }}$. In this context, we observe that the "perfection mutation" $\mathfrak{M}^{\mathrm{pf}}$ also yields a core - i.e., another "invariant piece" - of the Frobenius mutation-history. On the other hand, the natural morphism $\Phi_{S}: S \rightarrow S^{(p)}$ may be interpreted as a covariant observable of this mutation-history whose observation mutations are the identity mutations $\operatorname{id}_{\mathfrak{S}^{p \text {-sch }}}: \mathfrak{S}^{p \text {-sch }} \rightsquigarrow \mathfrak{S}^{p \text {-sch }}$. Since $\Phi_{S}$ is not, in general, an isomorphism, it follows that this observable constitutes an example of an non-coric observable. Nevertheless, the natural morphism $\beta_{S}: S^{\mathrm{pf}} \rightarrow S$ may be interpreted as a morphism of mutations $\mathfrak{M}^{\mathrm{pf}} \rightarrow \mathrm{id}_{\mathfrak{S}^{p-s c h}}$ that serves to relate the non-coric observable just considered to the coric observable arising from $\mathfrak{M}^{\mathrm{pf}}$.

(iii) One may also develop a version of (i), (ii) for log schemes; we leave the routine details to the interested reader. Here, we pause to mention that the theory of log schemes motivates the following "combinatorial monoid-theoretic" version of the non-coric observable on the Frobenius mutation-history of (ii). Write 
for the species of torsion-free abelian monoids and morphisms of monoids. If $M$ is a species-object of $\mathfrak{S}^{\text {mon }}$, then write $M^{(p)} \stackrel{\text { def }}{=} p \cdot M \subseteq M$. Then the assignment $M \mapsto M^{(p)}$ determines a "monoid-Frobenius mutation"

$$
\mathfrak{F}^{\text {mon }}: \mathfrak{S}^{\text {mon }} \rightsquigarrow \mathfrak{S}^{\text {mon }}
$$

and hence a "monoid-Frobenius mutation-history"

$$
\ldots \rightsquigarrow \mathfrak{S}^{\text {mon }} \rightsquigarrow \mathfrak{S}^{\text {mon }} \rightsquigarrow \ldots
$$

which is equipped with a non-coric contravariant observable determined by the natural inclusion morphism $M^{(p)} \hookrightarrow M$ and the observation mutations given by the identity mutations $\operatorname{id}_{\mathfrak{S}^{\text {mon }}}: \mathfrak{S}^{\text {mon }} \rightsquigarrow \mathfrak{S}^{\text {mon }}$. On the other hand, the p-perfection $M^{\text {pf }}$ of $M$, i.e., the inductive limit of the inductive system $M \hookrightarrow M \hookrightarrow M \hookrightarrow \ldots$ obtained by considering the inclusions given by multiplying by $p$, gives rise to a "monoid-p-perfection mutation"

$$
\mathfrak{F}^{\text {pf }}: \mathfrak{S}^{\text {mon }} \rightsquigarrow \mathfrak{S}^{\text {mon }}
$$

which may be interpreted as a core of the monoid-Frobenius mutation-history. Finally, the natural inclusion of monoids $M \hookrightarrow M^{\text {pf }}$ may be interpreted as a morphism of mutations $\mathrm{id}_{\mathfrak{S}^{\text {mon }}} \rightarrow \mathfrak{F}^{\mathrm{pf}}$ that serves to relate the non-coric observable just considered to the coric observable arising from $\mathfrak{F}^{\mathrm{pf}}$.

\section{Remark 3.6.1.}

(i) The various constructions of Example 3.6 may be thought of as providing, in the case of the phenomena of "invariance of the étale site under Frobenius" and "invariance of the perfection under Frobenius", a "species-theoretic intepretation" — i.e., via consideration of

\section{"coric" versus "non-coric" observables}

— of the difference between "étale-type" and "Frobenius-type" structures [cf. the discussion of [FrdI], §I4]. This sort of approach via "combinatorial patterns" to expressing the difference between "étale-type" and "Frobenius-type" structures plays a central role in the theory of the present series of papers. Indeed, the mutation-histories and cores considered in Example 3.6, (ii), (iii), may be thought of as the underlying motivating examples for the theory of both

- the vertical lines, i.e., consisting of log-links, and

· the horizontal lines, i.e., consisting of $\Theta^{\times \mu}-/ \Theta_{\text {gau }}^{\times \mu}-/ \Theta_{\mathrm{LGP}}^{\times \mu}-/ \Theta_{\mathfrak{l g p}}^{\times \mu}-$ links,

of the log-theta-lattice [cf. [IUTchIII], Definitions 1.4, 3.8]. Finally, we recall that this approach to understanding the log-links may be seen in the introduction of the terminology of "observables" and "cores" in [AbsTopIII], Definition 3.5, (iii).

(ii) Example 3.6 also provides a good example of the important theme [cf. the discussion of Remark 3.3.1, (iii)] of 
describing non-coric data in terms of coric data

— cf. the morphism $\beta_{S}: S^{\mathrm{pf}} \rightarrow S$ of Example 3.6, (ii); the natural inclusion $M \hookrightarrow M^{\text {pf }}$ of Example 3.6, (iii). From the point of view of the vertical and horizontal lines of the log-theta-lattice [cf. the discussion of (i)], this theme may also be observed in the vertically coric log-shells that serve as a common receptacle for the various arrows of the log-Kummer correspondences of [IUTchIII], Corollary 3.11, (ii), as well as in the multiradial representations of [IUTchIII], Corollary 3.11, (i), which describe [certain aspects of] the arithmetic holomorphic structure on one vertical line of the log-theta-lattice in terms that may be understood relative to an alien arithmetic holomorphic structure on another vertical line - i.e., separated from the first vertical line by horizontal arrows - of the log-theta-lattice [cf. [IUTchIII], Remark 3.11.1; [IUTchIII], Remark 3.12.3, (ii)].

\section{Remark 3.6.2.}

(i) In the context of the theme of "coric descriptions of non-coric data" discussed in Remark 3.6.1, (ii), it is of interest to observe the significance of the use of set-theoretic formulas [cf. the discussion of Remarks 3.1.2, 3.1.3, 3.1.4] to realize such descriptions. That is to say, descriptions in terms of arbitrary choices that depend on a particular model of set theory [cf. Remark 3.1.3] do not allow one to calculate in terms that make sense in one universe the operations performed in an alien universe! This is precisely the sort of situation that one encounters when one considers the vertical and horizontal arrows of the log-theta-lattice [cf. (ii) below], where distinct universes arise from the distinct scheme-theoretic basepoints on either side of such an arrow that correspond to distinct ring theories, i.e., ring theories that cannot be related to one another by means of a ring homomorphism — cf. the discussion of Remark 3.6.3 below. Indeed,

it was precisely the need to understand this sort of situation that led the author to develop the "inter-universal" version of Teichmüller theory exposed in the present series of papers.

Finally, we observe that the algorithmic approach [i.e., as opposed to the "fully faithfulness/Grothendieck Conjecture-style approach" — cf. Example 3.5, (iii)] to reconstruction issues via set-theoretic formulas plays an essential role in this context. That is to say, although different algorithms, or software, may yield the same output data, it is only by working with specific algorithms that one may understand the delicate inter-relations that exist between various components of the structures that occur as one performs various operations [i.e., the mutations of a mutation-history]. In the case of the theory developed in the present series of papers, one central example of this phenomenon is the cyclotomic rigidity isomorphisms that underlie the theory of $\Theta_{\mathrm{LGP}}^{\times \boldsymbol{\mu}}$-link compatibility discussed in [IUTchIII], Corollary 3.11, (iii), (c), (d) [cf. also [IUTchIII], Remarks 2.2.1, 3.10.1].

(ii) The algorithmic approach to reconstruction that is taken throughout the present series of papers, as well as, for instance, in [FrdI], [EtTh], and [AbsTopIII], was conceived by the author in the spirit of the species-theoretic formulation exposed in the present $§ 3$. Nevertheless, [cf. Remark 3.1.3, (i)] we shall not explicitly 
write out the various set-theoretic formulas involved in the various species, mutations, etc. that are implicit throughout the theory of the present series of papers. Rather, it is to be understood that the set-theoretic formulas to be used are those arising from the conventional descriptions that are given of the mathematical objects involved. When applying such conventional descriptions, however, the reader is obliged to check that they are well-defined and do not depend upon the use of arbitrary choices that are not describable via well-defined set-theoretic formulas.

(iii) The sharp contrast between

- the canonicality imparted by descriptions via set-theoretic formulas in the context of extensions of the universe in which one works

[cf. Remarks 3.1.3, 3.1.4] and

- the situation that arises if one allows, in one's descriptions, the various arbitrary choices arising from invocations of the axiom of choice

may be understood somewhat explicitly if one attempts to "catalogue the various possibilites" corresponding to various possible choices that may occur in one's description. That is to say, such a "cataloguing operation" typically obligates one to work with "sets of very large cardinality", many of which must be constructed by means of set-theoretic exponentiation [i.e., such as the operation of passing from a set $E$ to the set " 2 " " of all subsets of $E]$. Such a rapid outbreak of "unwieldy large sets" is reminiscent of the rapid growth, in the $p$-adic crystalline theory, of the $p$-adic valuations of the denominators that occur when one formally integrates an arbitrary connection, as opposed to a "canonical connection" of the sort that arises from a crystalline representation. In the $p$-adic theory, such "canonical connections" are typically related to "canonical liftings", such as, for instance, those that occur in $\boldsymbol{p}$-adic Teichmüller theory [cf. [ $p$ Ord], [ $p$ Teich $]]$. In this context, it is of interest to recall that the canonical liftings of $p$-adic Teichmüller theory may, under certain conditions, be thought of as liftings "of minimal complexity" in the sense that their Witt vector coordinates are given by polynomials of minimal degree - cf. the computations of [Finot].

\section{Remark 3.6.3.}

(i) In the context of Remark 3.6.2, it is useful to recall the fundamental reason for the need to pursue "inter-universality" in the present series of papers [cf. the discussion of [IUTchIII], Remark 1.2.4; [IUTchIII], Remark 1.4.2], namely,

since étale fundamental groups - i.e., in essence, Galois groups - are defined as certain automorphism groups of fields/rings, the definition of such a Galois group as a certain automorphism group of some ring structure is fundamentally incompatible with the vertical and horizontal arrows of the log-theta-lattice [i.e., which do not arise from ring homomorphisms]!

In this respect, "transformations" such as the vertical and horizontal arrows of the log-theta-lattice differ, quite fundamentally, from "transformations" that are 
compatible with the ring structures on the domain and codomain, i.e., morphisms of rings/schemes, which tautologically give rise to functorial morphisms between the respective étale fundamental groups. Put another way, in the notation of [IUTchI], Definition 3.1, (e), (f), for, say, $\underline{v} \in \underline{\mathbb{V}}^{\text {non }}$,

the only natural correspondence that may be described by means of settheoretic formulas between the isomorphs of the local base field Galois groups " $G_{\underline{v}}$ " on either side of a vertical or horizontal arrow of the log-theta-lattice is the correspondence constituted by an indeterminate isomorphism of topological groups.

A similar statement may be made concerning the isomorphs of the geometric fundamental group $\Delta_{\underline{v}} \stackrel{\text { def }}{=} \operatorname{Ker}\left(\Pi_{\underline{v}} \rightarrow G_{\underline{v}}\right)$ on either side of a vertical [but not horizontal! - cf. the discussion of (ii) below] arrow of the log-theta-lattice - that is to say,

the only natural correspondence that may described by means of settheoretic formulas between these isomorphs is the correspondence constituted by an indeterminate isomorphism of topological groups equipped with some outer action by the respective isomorph of " $G_{\underline{v}}$ "

— cf. the discussion of [IUTchIII], Remark 1.2.4. Here, again we recall from the discussion of Remark 3.6.2, (i), (ii), that it is only by working with such correspondences that may be described by means of set-theoretic formulas that one may obtain descriptions that allow one to calculate the operations performed in one universe from the point of view of an alien universe.

(ii) One fundamental difference between the vertical and horizontal arrows of the $\log$-theta-lattice is that whereas, for, say, $\underline{v} \in \underline{\mathbb{V}}^{\text {bad }}$,

(V1) one identifies, up to isomorphism, the isomorphs of the full arithmetic fundamental group " $\Pi_{\underline{v}}$ " on either side of a vertical arrow,

(H1) one distinguishes the " $\Delta_{\underline{v}}$ 's" on either side of a horizontal arrow, i.e., one only identifies, up to isomorphism, the local base field Galois groups " $G_{\underline{\underline{v}}}$ " on either side of a horizontal arrow.

- cf. the discussion of [IUTchIII], Remark 1.4.2. One way to understand the fundamental reason for this difference is as follows.

(V2) In order to construct the log-link - i.e., at a more concrete level, the power series that defines the $p_{\underline{v}}$-adic logarithm at $\underline{v}$ - it is necessary to avail oneself of the local ring structures at $\underline{v}$ [cf. the discussion of [IUTchIII], Definition 1.1, (i), (ii)], which may only be reconstructed from the full " $\Pi_{\underline{v}}$ " [i.e., not from " $G_{\underline{v}}$ stripped of its structure as a quotient of $\Pi_{\underline{v}}$ " - cf. the discussion of [IUTTchIII], Remark 1.4.1, (i); [IUTchIII], Remark 2.1.1, (ii); [AbsTopIII], §I3].

(H2) In order to construct the $\Theta_{\mathrm{gau}^{-}}^{\times \boldsymbol{\mu}}-/ \Theta_{\mathrm{LGP}}^{\times \boldsymbol{\mu}}-/ \Theta_{\mathfrak{l g p}^{\prime}}^{\times \boldsymbol{\mu}}$-links - i.e., at a more concrete level, the correspondence

$$
\underline{\underline{q}} \mapsto\left\{\underline{\underline{q}}^{j^{2}}\right\}_{j=1, \ldots, l *}
$$


[cf. [IUTchII], Remark 4.11.1] — it is necessary, in effect, to construct an "isomorphism" between a mathematical object [i.e., the theta values " $\underline{q}^{j}$ "] that depends, in an essential way, on regarding the various " $j$ " as distinct labels [which are constructed from " $\Delta_{v}$ "!] and a mathematical object [i.e., "q"] that is independent of these labels; it is then a tautology that such an "isomorphism" may only be achieved if the labels - i.e., in essence, " $\Delta_{\underline{v}}$ " — on either side of the "isomorphism" are kept distinct from one another.

Here, we observe in passing that the "apparently horizontal arrow-related" issue discussed in (H2) of simultaneous realization of "label-dependent" and "labelfree" mathematical objects is reminiscent of the vertical arrow portion of the bicoricity theory of [IUTchIII], Theorem 1.5 - cf. the discussion of [IUTchIII], Remark 1.5.1, (i), (ii); Step (vii) of the proof of [IUTchIII], Corollary 3.12. 


\section{Bibliography}

[NerMod] S. Bosch, W. Lütkebohmert, M. Raynaud, Néron Models, Ergebnisse der Mathematik und ihrer Grenzgebiete 21, Springer-Verlag (1990).

[Drk] F. R. Drake, Set Theory: an Introduction to Large Cardinals, Studies in Logic and the Foundations of Mathematics 76, North-Holland (1974).

[DmMn] H. Dym and H. P. McKean, Fourier Series and Integrals, Academic Press (1972).

[Edw] H. M. Edwards, Riemann's Zeta Function, Academic Press (1974).

[Ffmn] S. Feferman, Set-theoretical Foundations of Category Theory, Reports of the Midwest Category Seminar III, Lecture Notes in Mathematics 106, SpringerVerlag (1969), pp. 201-247.

[Finot] L. R. A. Finotti, Minimal degree liftings of hyperelliptic curves, J. Math. Sci. Univ. Tokyo 11 (2004), pp. 1-47.

[FK] E. Freitag and R. Kiehl, Étale Cohomology and the Weil Conjecture, Springer Verlag (1988).

[Harts] R. Hartshorne, Algebraic Geometry, Graduate Texts in Mathematics 52, SpringerVerlag (1977).

[Kobl] N. Koblitz, p-adic Numbers, p-adic Analysis, and Zeta-Functions, Graduate Texts in Mathematics 58, Springer-Verlag (1984).

[McLn] S. MacLane, One Universe as a Foundation for Category Theory, Reports of the Midwest Category Seminar III, Lecture Notes in Mathematics 106, SpringerVerlag (1969).

[Mss] D. W. Masser, Note on a conjecture of Szpiro in Astérisque 183 (1990), pp. 19-23.

[Milne] J. S. Milne, Abelian Varieties in Arithmetic Geometry, edited by G. Cornell and J. H. Silverman, Springer-Verlag (1986), pp. 103-150.

[pOrd] S. Mochizuki, A Theory of Ordinary p-adic Curves, Publ. Res. Inst. Math. Sci. 32 (1996), pp. 957-1151.

[pTeich] S. Mochizuki, Foundations of p-adic Teichmüller Theory, AMS/IP Studies in Advanced Mathematics 11, American Mathematical Society/International Press (1999).

[HASurI] S. Mochizuki, A Survey of the Hodge-Arakelov Theory of Elliptic Curves I, Arithmetic Fundamental Groups and Noncommutative Algebra, Proceedings of Symposia in Pure Mathematics 70, American Mathematical Society (2002), pp. 533-569.

[HASurII] S. Mochizuki, A Survey of the Hodge-Arakelov Theory of Elliptic Curves II, Algebraic Geometry 2000, Azumino, Adv. Stud. Pure Math. 36, Math. Soc. Japan (2002), pp. 81-114. 
[CanLift] S. Mochizuki, The Absolute Anabelian Geometry of Canonical Curves, Kazuya Kato's fiftieth birthday, Doc. Math. 2003, Extra Vol., pp. 609-640.

[LgSch] S. Mochizuki, Categorical representation of locally noetherian log schemes, Adv. Math. 188 (2004), pp. 222-246.

[ArLgSch] S. Mochizuki, Categories of log schemes with Archimedean structures, J. Math. Kyoto Univ. 44 (2004), pp. 891-909.

[SemiAnbd] S. Mochizuki, Semi-graphs of Anabelioids, Publ. Res. Inst. Math. Sci. 42 (2006), pp. 221-322.

[Cusp] S. Mochizuki, Absolute anabelian cuspidalizations of proper hyperbolic curves, J. Math. Kyoto Univ. 47 (2007), pp. 451-539.

[FrdI] S. Mochizuki, The Geometry of Frobenioids I: The General Theory, Kyushu J. Math. 62 (2008), pp. 293-400.

[EtTh] S. Mochizuki, The Étale Theta Function and its Frobenioid-theoretic Manifestations, Publ. Res. Inst. Math. Sci. 45 (2009), pp. 227-349.

[GenEll] S. Mochizuki, Arithmetic Elliptic Curves in General Position, Math. J. Okayama Univ. 52 (2010), pp. 1-28.

[AbsTopIII] S. Mochizuki, Topics in Absolute Anabelian Geometry III: Global Reconstruction Algorithms, RIMS Preprint 1626 (March 2008).

[IUTchI] S. Mochizuki, Inter-universal Teichmüller Theory I: Construction of Hodge Theaters, preprint.

[IUTchII] S. Mochizuki, Inter-universal Teichmüller Theory II: Hodge-Arakelov-theoretic Evaluation, preprint.

[IUTchIII] S. Mochizuki, Inter-universal Teichmüller Theory III: Canonical Splittings of the Log-theta-lattice, preprint.

[Silv] J. H. Silverman, The Arithmetic of Elliptic Curves, Graduate Texts in Mathematics 106, Springer-Verlag (1986).

[Arak] C. Soulé, D. Abramovich, J.-F. Burnol, J. Kramer, Lectures on Arakelov Geometry, Cambridge studies in advanced mathematics 33, Cambridge University Press (1994).

[Vjt] P. Vojta, Diophantine approximations and value distribution theory, Lecture Notes in Mathematics 1239, Springer-Verlag (1987). 